\title{
THE IMPLICATIONS OF SUPPLIER ENCROACHMENT VIA AN ONLINE PLATFORM
}

\author{
Feng Fu ${ }^{1}$, Shuangying $\mathrm{CHEN}^{2, *}$ And WeI YAN ${ }^{2}$
}

\begin{abstract}
E-commerce provides suppliers with the flexibility to operate an online arm via a platform in addition to their pre-existing physical stores. Although such supplier encroachment is becoming increasingly prevalent in e-commerce markets, the literature on supplier encroachment traditionally assumes that suppliers sell products to consumers directly and argues that supplier encroachment can mitigate double marginalisation problems that can secure Pareto improvements. This paper narrows this gap by investigating the implications of the supplier encroachment with an online platform under two scenarios (i.e., the platform owner forgoing or retaining its entry options). A central result obtained is that, unlike supplier-owned direct channels and in addition to the "win-win" outcomes for the supplier and the traditional retailer, supplier encroachment with an online platform may also lead to "winlose" and "lose-lose" outcomes. Furthermore, when the platform owner retains its entry option, such encroachment is always detrimental for the traditional retailer but beneficial for the supplier.
\end{abstract}

Mathematics Subject Classification. 90Bxx.

Received November 9, 2020. Accepted January 3, 2022.

\section{INTRODUCTION}

Suppliers can encroach on the retail market in different ways, including supplier outlets, company-owned stores, catalogue sales, telemarketing, and online sales [3]. Over the last decade, with the development of evermore-sophisticated logistics and the rise of information technology [18], online retail platforms (for example, eBay, Amazon's Marketplace and OLX.in) have become major engines of growth in e-commerce [14]. As a result, a growing number of suppliers have found it attractive to supplement their preexisting retail channels with an online platform channel. For example, many brand-name suppliers, including Apple, Samsung, IBM, and Lenovo, have sold their products on Amazon as well as through stores such as Best Buy and Circuit City. Similarly, among independent sellers on the Alibaba Group's platform (http://www.taobao.com; referred to as Taobao for the rest of the paper) can be found many well-known suppliers in a variety of industries, such as Burberry (apparel), HP (electronics and appliances) and Audi (automobiles), who have opened flagship company stores on this online platform.

Keywords. Operations, E-commerce, retail, distribution channels, game theory.

1 School of Business, Chengdu University of Technology, Chengdu 610059, P.R. China.

2 School of Management and Economics, University of Electronic Science and Technology of China, Chengdu 61173, P.R. China.

* Corresponding author: shychen@uestc.edu.cn 
Supplier encroachment with an online platform occurs in two basic forms. In the first, online retail platforms, such as Taobao, offer suppliers or sales agents direct access to consumers for a fee, while the platform owner forgoes its entry option. In the second, the platform owner retains the option to resell the products from the supplier with an additional allowance for the supplier or sale agent, such as Amazon. In the last decade, these selling formats have become pervasive, facilitated by online retail platforms [27]. For instance, Amazon directly sells $7 \%$ of the products in its electronics category, and the remaining $93 \%$ of the products are sold by independent sellers [20]. In the fourth quarter of 2016, third-party transactions accounted for $49 \%$ of all unit sales on Amazon [35]. This scenario is similar for Taobao. As the world's largest online consumer-to-consumer platform based in China, Taobao has over seven million sellers less than a decade after its establishment in 2003 [14].

Numerous researchers $[3,8,11,17,40]$ have addressed supplier encroachment on the assumption that suppliers sell products through a supplier-owned direct channel, thus ignoring the role played by the online platform and its impact on the traditional retailer, online pricing strategies and profits. Moreover, it has long been recognised that supplier encroachment has strategic consequences on sustainable operations. When the supplier encroaches on the retail market, she has an incentive to reduce the wholesale price to not unduly diminish the traditional retailer's demand. As a result, supplier encroachment can create a "win-win" outcome for the supplier and the traditional retailer $[3,24,36,40]$. However, the online platform introduces another dimension to the relationship between suppliers and traditional retailers that needs to be addressed. Specifically, if the supplier sells her products through an online platform, the platform owner not only determines its per-unit fee but also ascertains whether to forgo its entry option or not. Furthermore, if the platform owner retains its entry option, then the supplier needs to determine wholesale prices for the traditional retailer and the platform owner, respectively.

Although there has been a rich body of work on platform-based markets, beginning with Rochet and Tirole [32], prior work in this area has primarily focused on platform competition $[1,6,21]$, pricing structure $[4,20,25,37]$ and strategic decisions $[5,14,19,31]$ and less on the interaction between online retail platforms and offline traditional retail channels. This interaction poses several questions that differ from those around supplierowned channels and traditional retail channels. For example, when facing supplier encroachment with an online platform, a retailer in the traditional channel may be in a more challenging position than when confronting a supplier-owned channel. This is because he is not only essentially competing with the products from the supplier, but also with the products that are resold by the online platform.

In this paper, the focus is on supplier encroachment via an online platform. Specifically, a model is developed describing how a supplier encroaches on the retail market by selling products through an online retail platform. We intend to answer the following questions: What are the implications of supplier encroachment with an online platform for the supplier, the traditional retailer and the platform owner? In particular, when the platform owner retains its entry option, should the supplier encroach on the retail market? Finally, how does supplier encroachment with an online platform create strategic issues that differ significantly from those with supplierowned channels?

These strategic interactions are studied and novel insights into supplier encroachment are provided. The main result is in contrast to those of Arya et al. [3]: in addition to "win-win" outcomes for the supplier and traditional retailer, supplier encroachment with an online platform may also lead to "win-lose" and "lose-lose" outcomes. In particular, when the platform owner retains its entry option, supplier encroachment is always beneficial to the supplier but detrimental to the traditional retailer; as a result, "win-lose" is the only possible outcome for the supplier and traditional retailer.

The model developed contributes to the literature in several important ways. First, an aspect of supplier encroachment mostly ignored by the extant research is addressed. Unlike prior studies, which examine scenarios in which the supplier encroaches on the retail market with a supplier-owned direct channel, this study highlights the fact that suppliers can encroach on the retail market by selling products through an online platform. It focuses on the strategic issues created by supplier encroachment with an online platform, which differ significantly from those resulting from a supplier-owned channel. Second, although the question of whether supplier encroachment 
results in "internet channel conflict" or brings Pareto gains to both parties has been well studied in dualchannel supply chains, aside from the traditional retailer's cost advantage, there is limited knowledge about how the role played by online platforms can affect these results. Third, although there is a rich body of work on platform-based markets, limited attention has been paid to the interaction between online retail platforms and offline traditional retail channels. Therefore, this paper sheds new light on a setting where a supplier encroaches on the retail market by selling products through an online platform - an increasingly prevalent occurrence in e-commerce markets.

The remainder of the paper is organised as follows. Section 2 reviews related literature and explains the contributions of this paper more thoroughly. Section 3 describes the key elements of four models. Section 4 presents our central findings. Section 5 extends the analysis to the simultaneous setting. Section 6 concludes the paper.

\section{Related Literature}

This work lies at the intersection of supplier encroachment and platform-based business models, where the former mainly focuses on the implications of the supplier-owned direct channel on the supplier and traditional retailer, and the latter examines various aspects associated with platform-based markets grounded in the literature on industrial economics and two-sided markets. To clearly delineate the contributions of this paper, each of these literature streams is discussed.

Most research on supplier encroachment has taken one of two familiar approaches. The first emphasises that supplier encroachment can lead to a "win-win" outcome for the supplier and the traditional retailer (see, e.g., $[7,13,38,40])$. In particular, Arya et al. $[3]$ show that supplier encroachment not only creates additional profits for the supplier, but also motivates her to set a lower wholesale price in the encroachment setting than in the no-encroachment setting to offset the advantage that the supplier's retail arm secures. As a result, supplier encroachment should create a "win-win" outcome for the supplier and the traditional retailer because it induces lower wholesale prices to support the wholesale demand of the weakened incumbent retailer. It should be noted that the pioneering literature generally assumes that the supplier encroaches into the retail market through a supplier-owned direct channel. However, platform-based business models have become a major engine of growth in e-commerce [14]. As mentioned earlier, from a research perspective, the forms of these platform-based business models are quite different from those of supplier-owned direct channels. For example, platform owners allow suppliers or sales agents direct access to consumers, charging them only for value-added services. Moreover, with the addition of an allowance for the supplier or sale agent, the online retail platforms may retain the option to resell the products from the supplier. As such, we extend the analysis in Arya et al. [3] to address the implications of supplier encroachment via an online platform on the traditional retailer, online pricing strategies, and profits. As noted by Arya et al. [3], when the supplier encroaches on the retail market by selling products directly, she has an incentive to reduce the wholesale price to avoid unduly diminishing the traditional retailer's demand for the supplier's wholesale products, which can lead to a "win-win" outcome for both parties. Our analysis demonstrates, however, that it is not always true under the supplier encroachment scenario with an online platform. In particular, when the platform owner is endowed with the option of entering the market, the supplier encroachment is always beneficial for the supplier but detrimental to the traditional retailer.

This research stream has recently moved beyond these initial fundamental analyses and considered how the robustness of the "win-win" outcome changes with some assumptions. For example, a few studies have extended supplier encroachment to scenarios with asymmetric information [23], nonlinear pricing [24], and endogenous quality $[12,17]$, revealing that, under certain conditions, supplier encroachment may always be detrimental to the traditional retailer and may lead to a "lose-lose" situation for the supplier and traditional retailer. Our work complements this stream of research by addressing supplier encroachment with an online platform, leading to results that differ from the current literature. They indicate that there is an alliance between the supplier and the online platform when the platform owner retains its encroachment option. 
The second research stream is related to the literature on platform-based markets, which is grounded in the literature on industrial economics and two-sided markets $[28,34]$. Although there is a rich body of work on platform economies and two-sided markets - beginning with Rochet and Tirole [32] - prior work on online retail platforms has primarily focused on platform competition [1,6,21], pricing structure $[4,20,25,37]$ and strategic decisions by platforms beyond pricing $[5,14,19,31]$. They have focused less on the interaction between online retail platforms and offline traditional retail channels. The current work complements this stream of research by investigating the implications of supplier encroachment with an online platform under two scenarios (i.e., the platform owner forgoing or retaining its entry options). Specifically, we develop two basic forms of supplier encroachment with an online platform. In the first, the supplier encroaches on the retail market via an online platform who forgoes its option to resell the products from the supplier. In the second, the platform owner allows the supplier access to consumers through the online retail platform for a fee, while retaining its entry option.

\section{THE MODEL}

This section introduces the notation and lays out assumptions regarding the four models for the product, supplier, traditional retailer, platform owner, and consumers. We describe each in detail below.

\subsection{Formulation}

As shown in Figure 1, we set up four models. First, we established Model N and Model EM with the platform owner forgoing its entry option. In Model N (benchmark case), all products are distributed only through the traditional retail channel. In Model EM, the supplier encroaches on the retail market through the online platform. Subsequently, we provided Model ET and Model EB with the platform owner retaining its entry option. In Model ET, the supplier wholesales products to the traditional retailer and platform owner without selling the product directly to consumers. However, in Model EB, both the supplier and platform owner encroach on the traditional retailer's market.

Let $\pi_{i}^{j}$ represent the profit of party $i$ under model $j$, where $i=s, r, p$ refers to the supplier, traditional retailer, and platform owner, while $j=\mathrm{N}, \mathrm{EM}, \mathrm{ET}, \mathrm{EB}$ refers to Model N, Model EM, Model ET and Model $\mathrm{EB}$, respectively. The timeline is as follows (in Fig. 2): the supplier announces the wholesale price to the traditional retailer and platform owner simultaneously (when the platform retains its entry option), then the traditional retailer responds by determining the optimal units. Subsequently, the platform owner selects a perunit fee for each sale of the supplier, and then it announces the optimal units sold to consumers (when the platform retains its entry option). Meanwhile, the supplier chooses the optimal quantities of products to be sold on the online platform ${ }^{1}$.

Like Arya et al. [3] and Xiong et al. [40], we adopt a single market-clearing price to preclude price discrimination. That is, we assume that consumers have no preference between the online retail platform and traditional retail channel, and their demand for the product is represented by a linear, downward-sloping (inverse) demand, $p=1-Q$, where $p$ and $Q=q_{r}+q_{s}$ are the price and quantities of the product, respectively.

We let $c_{p}$ and $c_{r}$ reflect the differences in selling costs of online and traditional channels, which are uniformly distributed in the interval of $[0,1]$. Note that, if $c_{r}<c_{p}$, the traditional retailer's selling costs may be less compared to the online platform, so it has an advantage in product distribution; otherwise, the opposite is true $^{2}$. Such a difference in selling costs has been widely adopted in the literature in marketing to reflect the level of competition between both channels $[3,9,17]$.

In practice, to compete with traditional retailers, many major online platforms, such as Amazon and JD.com, have invested heavily in their marketing efforts (e.g., using novel virtual reality technologies, social and online advertising, and attractive delivery times) that alleviate the consumers' disutility from online transactions.

\footnotetext{
${ }^{1}$ This consequence of events is consistent with the assumption of supplier encroachment via online channel in Arya et al. [3]. And this assumption is relaxed in Section 5.

${ }^{2}$ We thank an anonymous reviewer for pointing this out.
} 


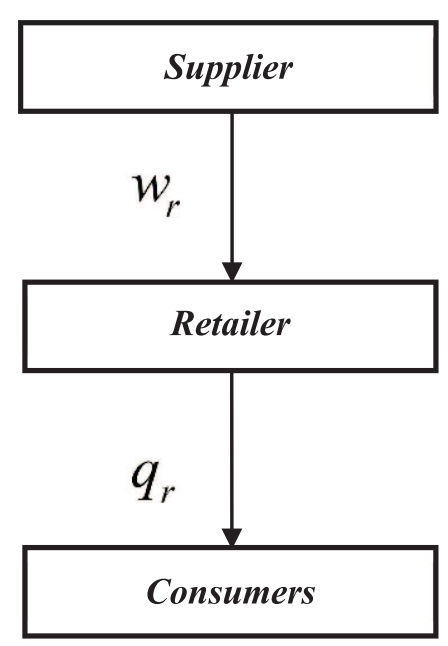

(a)

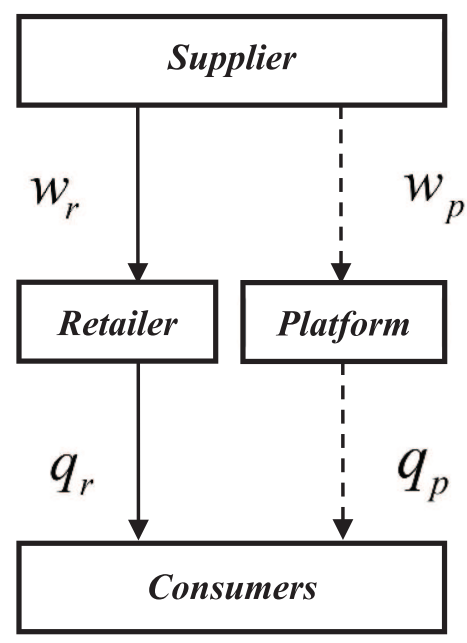

(c)

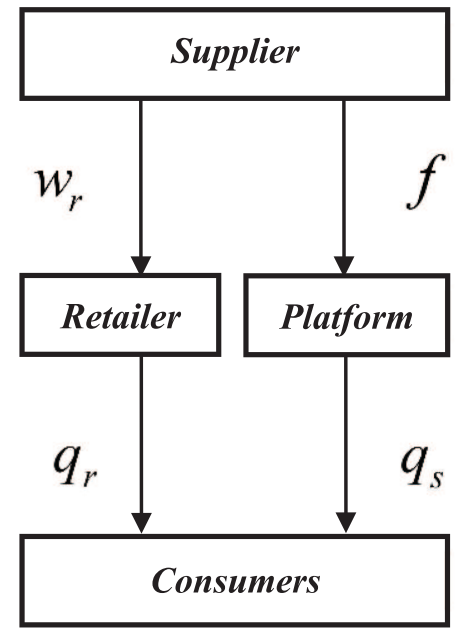

(b)

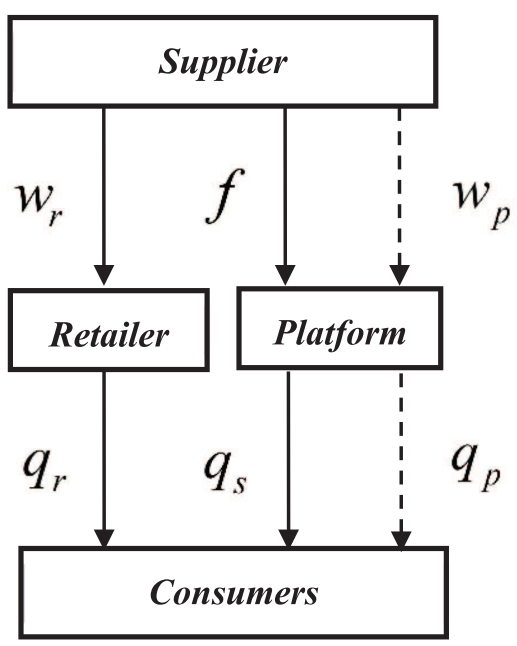

(d)

FiguRE 1. Four basic models for supplier encroachment with an online platform. (a) Model N. (b) Model EM. (c) Model ET. (d) Model EB.

Thus, the platform owner's performance is modelled as a function of the demand for products (i.e., $\left.q_{s}\right)$ that is influenced by its marketing effort, denoted by $I$, the investment in marketing efficiency. To characterise the diminishing returns on investment, similar to Savaskan et al. [33] and Jiang et al. [20], the cost structure $q_{s}=\sqrt{I / k}$ is used, where $k$ is a scaling parameter that shows the marketing efficiency for the platform owner to give consumers an incentive or enthusiasm for purchasing products in its marketplace. To differentiate from cross-investment to other partners, it is assumed that the platform owner can effectively influence its consumers through a self-investment, that is, $k \in(0,1)[2,16,39]$. It is natural to expect that, as the scaling parameter $k$ decreases, the online platform is more efficient in marketing a product, as the investment in finding effective 


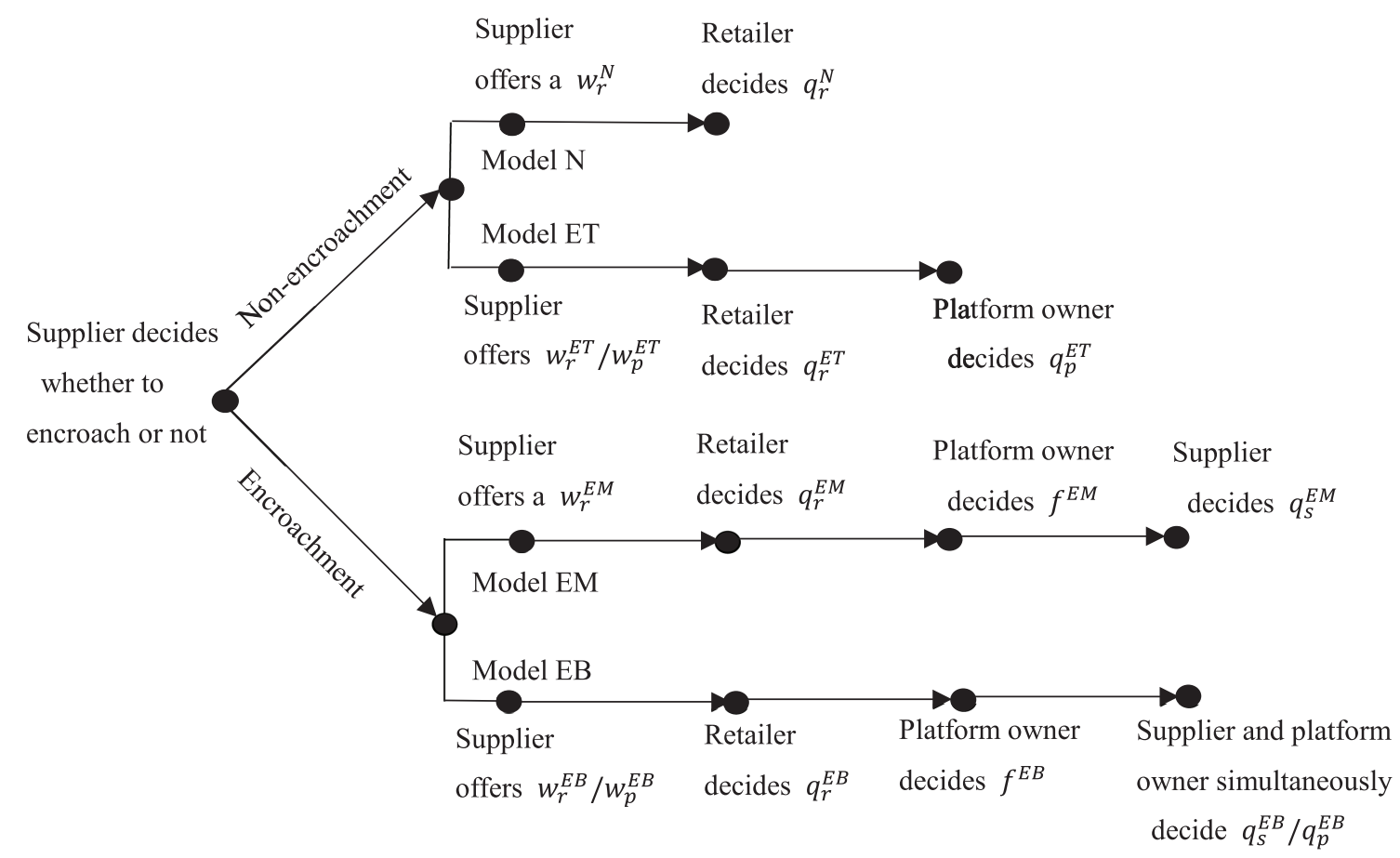

FIGURE 2. Timeline in the sequential setting.

demand or providing a service level is relatively small. Similar forms of response functions have widely been used in salesforce effort response models in the marketing literature [10,15, 22, 29].

\subsection{Equilibrium}

\subsubsection{Model N (Benchmark without encroachment)}

Here, we establish a benchmark-setting with no online retail platform channel, where all the products are distributed only through the traditional retail channel (Model $\mathrm{N}$ ). From the equation $p=1-Q$, the demand with the no encroachment setting is $p=1-q_{r}^{\mathrm{N}}$. In this model, the decisions for the supplier and traditional retailer are as follows.

$$
\begin{aligned}
& \pi_{s}^{\mathrm{N}}=w_{r}^{\mathrm{N}} q_{r}^{\mathrm{N}} \\
& \pi_{r}^{\mathrm{N}}=\left(1-q_{r}^{\mathrm{N}}-w_{r}^{\mathrm{N}}-c_{r}\right) q_{r}^{\mathrm{N}} .
\end{aligned}
$$

We use backward induction to solve this game. Following the timeline of Model $\mathrm{N}$ in Figure 1, for any given $w_{r}^{\mathrm{N}}$, the traditional retailer first chooses the equilibrium output $q_{r}^{\mathrm{N}}$ to maximise its profit $\pi_{r}^{\mathrm{N}}\left(w_{r}^{\mathrm{N}}, q_{r}^{\mathrm{N}}\right)$ :

$$
q_{r}^{\mathrm{N}^{*}}\left(w_{r}^{\mathrm{N}}\right)=\frac{1-w_{r}^{\mathrm{N}}-c_{r}}{2} .
$$

Substituting $q_{r}^{\mathrm{N}^{*}}\left(w_{r}^{\mathrm{N}}\right)$ into equation (3.1), the supplier determines the wholesale price to the traditional retailer $w_{r}^{\mathrm{N}}$ to maximise its profit $\pi_{s}^{\mathrm{N}}\left(w_{r}^{\mathrm{N}}, q_{r}^{\mathrm{N}^{*}}\left(w_{r}^{\mathrm{N}}\right)\right)$. Then, we can obtain the equilibrium wholesale prices, demand and profits, as summarised in Lemma $3.1^{3}$.

\footnotetext{
${ }^{3}$ For clarity, all proofs are provided in the Appendix A.
} 
Lemma 3.1. Under Model N, we have:

(i) The equilibrium wholesale prices and quantities are:

$$
q_{r}^{\mathrm{N}^{*}}=\frac{1-c_{r}}{4}, w_{r}^{\mathrm{N}^{*}}=\frac{1-c_{r}}{2} .
$$

(ii) The equilibrium profits of the supplier and traditional retailer are:

$$
\pi_{s}^{\mathrm{N}^{*}}=\frac{\left(1-c_{r}\right)^{2}}{8}, \pi_{r}^{\mathrm{N}^{*}}=\frac{\left(1-c_{r}\right)^{2}}{16}
$$

\subsubsection{Model EM}

In Model EM, the supplier would encroach into the retail market. The demand under this scenario is $p=$ $1-q_{r}^{\mathrm{EM}}-q_{s}^{\mathrm{EM}}$. Therefore, the profits for the supplier, traditional retailer and platform are as follows:

$$
\begin{aligned}
& \pi_{s}^{\mathrm{EM}}=\left(1-q_{r}^{\mathrm{EM}}-q_{s}^{\mathrm{EM}}-f^{\mathrm{EM}}\right) q_{s}^{\mathrm{EM}}+w_{r}^{\mathrm{EM}} q_{r}^{\mathrm{EM}} \\
& \pi_{r}^{\mathrm{EM}}=\left(1-q_{r}^{\mathrm{EM}}-q_{s}^{\mathrm{EM}}-w_{r}^{\mathrm{EM}}-c_{r}\right) q_{r}^{\mathrm{EM}} \\
& \pi_{P}^{\mathrm{EM}}=\left(f^{\mathrm{EM}}-c_{p}\right) q_{s}^{\mathrm{EM}}-k q_{s}^{\mathrm{EM}^{2}} .
\end{aligned}
$$

We use backward induction to solve this game. Following the timeline of Model EM in Figure 1, for any given $w_{r}^{\mathrm{EM}}, q_{r}^{\mathrm{EM}}$ and $f^{\mathrm{EM}}$, the supplier first chooses the direct sales to consumers $q_{s}^{\mathrm{EM}}$ to maximise its profit $\pi_{s}^{\mathrm{EM}}\left(w_{r}^{\mathrm{EM}}, q_{r}^{\mathrm{EM}}, f^{\mathrm{EM}}, q_{s}^{\mathrm{EM}}\right)$ :

$$
q_{s}^{\mathrm{EM}}\left(q_{r}^{\mathrm{EM}}, f^{\mathrm{EM}}\right)=\frac{1-q_{r}^{\mathrm{EM}}-f^{\mathrm{EM}}}{2} .
$$

When substituting $q_{s}^{\mathrm{EM}}{ }^{*}\left(q_{r}^{\mathrm{EM}}, f^{\mathrm{EM}}\right)$ into equation (3.6), the platform owner determines the commission fee $f^{\mathrm{EM}}$ to maximise its profit $\pi_{P}^{\mathrm{EM}}\left(f^{\mathrm{EM}}, q_{s}^{\mathrm{EM}}\left(q_{r}^{\mathrm{EM}}, f^{\mathrm{EM}}\right)\right)$ :

$$
f^{\mathrm{EM}^{*}}\left(q_{r}^{\mathrm{EM}}\right)=\frac{c_{p}+k-q_{r}^{\mathrm{EM}}-k q_{r}^{\mathrm{EM}}+1}{k+2} .
$$

Then, when substituting $q_{s}^{\mathrm{EM}^{*}}\left(q_{r}^{\mathrm{EM}}, f^{\mathrm{EM}}\right)$ and $f^{\mathrm{EM}^{*}}\left(q_{r}^{\mathrm{EM}}\right)$ into equation (3.5), the traditional retailer chooses equilibrium quantities $q_{r}^{\mathrm{EM}}$ to maximise its profit $\pi_{r}^{\mathrm{EM}}\left(w_{r}^{\mathrm{EM}}, q_{r}^{\mathrm{EM}}, q_{s}^{\mathrm{EM}^{*}}\left(q_{r}^{\mathrm{EM}}, f^{\mathrm{EM}}\right)\right)$ :

$$
q_{r}^{\mathrm{EM}}{ }^{*}\left(w_{r}^{\mathrm{EM}}\right)=\frac{3+c_{p}-4 c_{r}+2 k-2 c_{r} k-4 w_{r}^{\mathrm{EM}}-2 k w_{r}^{\mathrm{EM}}}{2(3+2 k)} .
$$

When substituting $q_{r}^{\mathrm{EM}^{*}}\left(w_{r}^{\mathrm{EM}}\right), f^{\mathrm{EM}^{*}}\left(q_{r}^{\mathrm{EM}}\right)$, and $q_{s}^{\mathrm{EM}^{*}}\left(q_{r}^{\mathrm{EM}}, f^{\mathrm{EM}}\right)$ into Equation (3.4), the supplier determines the wholesale price $w_{r}^{\mathrm{N}}$ to maximise its profit $\pi_{s}^{\mathrm{EM}}\left(w_{r}^{\mathrm{EM}}, q_{r}^{\mathrm{EM}}\left(w_{r}^{\mathrm{EM}}\right), f^{\mathrm{EM}^{*}}\left(q_{r}^{\mathrm{EM}}\right), q_{s}^{\mathrm{EM}^{*}}\left(q_{r}^{\mathrm{EM}}, f^{\mathrm{EM}}\right)\right)$. Then, we can obtain the equilibrium solutions, which are summarised in Lemma 3.2.

\section{Lemma 3.2. Under Model EM:}

(i) The equilibrium wholesale prices, quantities, and commission fee are:

$$
\begin{aligned}
w_{r}^{\mathrm{EM}^{*}} & =\frac{5 c_{p}-44 c_{r}+68 k+10 c_{p} k-78 c_{r} k+4 c_{p} k^{2}-44 c_{r} k^{2}-8 c_{r} k^{3}+40 k^{2}+8 k^{3}+39}{16 k^{3}+88 k^{2}+158 k+92} \\
q_{r}^{\mathrm{EM}^{*}} & =\frac{3 c_{p}-8 c_{r}+7 k+c_{p} k-8 c_{p} k-2 c_{r} k^{2}+2 k^{2}+5}{8 k^{2}+28 k+23} \\
f^{\mathrm{EM}^{*}} & =\frac{10 c_{p}+4 c_{r}+15 k+7 c_{p} k+6 c_{r} k+2 c_{r} k^{2}+6 k^{2}+9}{8 k^{2}+28 k+23} \\
q_{s}^{\mathrm{EM}^{*}} & =\frac{4 c_{r}-13 c_{p}+6 k-8 c_{p} k+2 c_{r} k+9}{2\left(8 k^{2}+28 k+23\right)}
\end{aligned}
$$


(ii) The equilibrium profits of the supplier, traditional retailer, and platform owner are:

$$
\begin{aligned}
\pi_{s}^{\mathrm{EM}^{*}} & =\frac{\left[\begin{array}{l}
9 c_{p}^{2} k+16 c_{p}^{2}-4 c_{p} c_{r} k^{2}-20 c_{p} c_{r} k-24 c_{p} c_{r}+4 c_{p} k^{2}+2 c_{p} k-8 c_{p}+4 c_{r}^{2} k^{3}+24 c_{r}^{2} k^{2} \\
+48 c_{r}^{2} k+32 c_{r}^{2}-8 c_{r} k^{3}-44 c_{r} k^{2}-76 c_{r} k-40 c_{r}+4 k^{3}+20 k^{2}+37 k+24
\end{array}\right]}{4\left(8 k^{3}+44 k^{2}+79 k+46\right)} \\
\pi_{r}^{\mathrm{EM}^{*}} & =\frac{(2 k+3)\left(3 c_{p}-8 c_{r}+7 k+c_{p} k-8 c_{r} k-2 c_{r} k^{2}+2 k^{2}+5\right)^{2}}{2\left(8 k^{2}+28 k+23\right)\left(8 k^{3}+44 k^{2}+79 k+46\right)} \\
\pi_{p}^{\mathrm{EM}^{*}} & =\frac{(k+2)\left(4 c_{r}-13 c_{p}+6 k-8 c_{p} k+2 c_{r} k+9\right)^{2}}{4\left(8 k^{2}+28 k+23\right)^{2}} .
\end{aligned}
$$

\subsubsection{Model ET}

In Model ET, the supplier wholesales products to the traditional retailer and platform owner without encroaching into the retail market. The demand under this scenario is $p=1-q_{r}^{\mathrm{ET}}-q_{P}^{\mathrm{ET}}$, where $q_{P}^{\mathrm{ET}}$ is the units sold by the platform owner. Thus, the profits for the supplier, traditional retailer and platform are as follows:

$$
\begin{aligned}
& \pi_{s}^{\mathrm{ET}}=w_{p}^{\mathrm{ET}} q_{p}^{\mathrm{ET}}+w_{r}^{\mathrm{ET}} q_{r}^{\mathrm{ET}} \\
& \pi_{r}^{\mathrm{ET}}=\left(1-q_{r}^{\mathrm{ET}}-q_{p}^{\mathrm{ET}}-w_{r}^{\mathrm{ET}}-c_{r}\right) q_{r}^{\mathrm{ET}} \\
& \pi_{P}^{\mathrm{EM}}=\left(1-q_{r}^{\mathrm{ET}}-q_{p}^{\mathrm{ET}}-w_{p}^{\mathrm{ET}}-c_{p}\right) q_{p}^{\mathrm{ET}}-k q_{p}^{\mathrm{ET}^{2}} .
\end{aligned}
$$

We use backward induction to solve this game. Following the timeline of Model ET in Figure 1, for any given $w_{r}^{\mathrm{ET}}, w_{P}^{\mathrm{ET}}$ and $q_{r}^{\mathrm{ET}}$, the platform owner first chooses the equilibrium quantities $q_{p}^{\mathrm{ET}}$ to maximise its profit $\pi_{p}^{\mathrm{ET}}\left(w_{p}^{\mathrm{ET}}, q_{r}^{\mathrm{ET}}, q_{p}^{\mathrm{ET}}\right)$ :

$$
q_{p}^{\mathrm{ET}}\left(w_{p}^{\mathrm{ET}}, q_{r}^{\mathrm{ET}}\right)=\frac{1-c_{p}-q_{r}^{\mathrm{ET}}-w_{p}^{\mathrm{ET}}}{2 k+2} .
$$

Then, when substituting $q_{p}^{\mathrm{ET}}{ }^{*}\left(w_{p}^{\mathrm{ET}}, q_{r}^{\mathrm{ET}}\right)$ into equation (3.11), the traditional retailer chooses equilibrium quantities $q_{r}^{\mathrm{ET}}$ to maximise its profit $\pi_{r}^{\mathrm{ET}}\left(w_{r}^{\mathrm{ET}}, q_{r}^{\mathrm{ET}}, q_{p}^{\mathrm{ET}}{ }^{*}\left(w_{p}^{\mathrm{ET}}, q_{r}^{\mathrm{ET}}\right)\right)$ :

$$
q_{r}^{\mathrm{ET}}\left(w_{r}^{\mathrm{ET}}, w_{p}^{\mathrm{ET}}\right)=\frac{c_{p}-2 c_{r}+2 k+w_{p}^{\mathrm{ET}}-2 w_{r}^{\mathrm{ET}}-2 c_{r} k-2 k w_{r}^{\mathrm{ET}}+1}{4 k+2} .
$$

When substituting $q_{p}^{\mathrm{ET}}\left(w_{p}^{\mathrm{ET}}, q_{r}^{\mathrm{ET}}\right)$ and $q_{r}^{\mathrm{ET}}\left(w_{p}^{\mathrm{ET}}, w_{r}^{\mathrm{ET}}\right)$ into equation (3.10), the supplier determines the wholesale price to the traditional retailer $w_{r}^{\mathrm{ET}}$ and the platform owner $w_{P}^{\mathrm{ET}}$ to maximise its profit $\pi_{s}^{\mathrm{ET}}\left(w_{p}^{\mathrm{ET}}, w_{r}^{\mathrm{ET}}, q_{p}^{\mathrm{ET}}\left(w_{p}^{\mathrm{ET}}, q_{r}^{\mathrm{ET}}\right), q_{r}^{\mathrm{ET}}{ }^{*}\left(w_{p}^{\mathrm{ET}}, w_{r}^{\mathrm{ET}}\right)\right)$ simultaneously. Then, we can obtain the equilibrium wholesale prices, demands and profits, as summarised in Lemma 3.3.

\section{Lemma 3.3. Under Model ET}

(i) The equilibrium wholesale prices and quantities are as follows:

$$
\begin{aligned}
w_{r}^{\mathrm{ET}} & =\frac{1-c_{r}}{2} \\
w_{P}^{\mathrm{ET}^{*}} & =\frac{1-c_{p}}{2} \\
q_{r}^{\mathrm{ET}} & =\frac{c_{p}-2 c_{r}+2 k-2 c_{r} k+1}{8 k+4} \\
q_{p}^{\mathrm{ET}^{*}} & =\frac{2 c_{r}-3 c_{p}+2 k-4 c_{p} k+2 c_{r} k+1}{16 k^{2}+24 k+8} .
\end{aligned}
$$


(ii) The equilibrium profits of the supplier, traditional retailer and platform owner are as follows:

$$
\begin{aligned}
\pi_{s}^{\mathrm{ET}} & =\frac{\left[\begin{array}{l}
4 c_{p}^{2} k+3 c_{p}^{2}-4 c_{p} c_{r} k-4 c_{p} c_{r}-4 c_{p} k-2 c_{p}+4 c_{r}^{2} k^{2}+8 c_{r}^{2} k+4 c r^{2} \\
-8 c_{r} k^{2}-12 c_{r} k-4 c_{r}+4 k^{2}+8 k+3
\end{array}\right]}{16\left(2 k^{2}+3 k+1\right)} \\
\pi_{r}^{\mathrm{ET}^{*}} & =\frac{\left(c_{p}-2 c_{r}+2 k-2 c_{r} k+1\right)^{2}}{32\left(2 k^{2}+3 k+1\right)} \\
\pi_{p}^{\mathrm{ET}^{*}} & =\frac{\left(2 c_{r}-3 c_{p}+2 k-4 c_{p} k+2 c_{r} k+1\right)^{2}}{64(2 k+1)^{2}(k+1)} .
\end{aligned}
$$

\subsubsection{Model EB}

In Model EB, both the supplier and platform owner encroach into the traditional retailer's market. The inverse demand function for the products is $p=1-q_{r}^{\mathrm{EB}}-q_{s}^{\mathrm{EB}}-q_{p}^{\mathrm{EB}}$. Thus, under this scenario, the supplier, traditional retailer and platform owner's decisions are as follows, respectively:

$$
\begin{aligned}
& \pi_{s}^{\mathrm{EB}}=\left(1-q_{r}^{\mathrm{EB}}-q_{s}^{\mathrm{EB}}-q_{p}^{\mathrm{EB}}-f^{\mathrm{EB}}\right) q_{s}^{\mathrm{EB}}+w_{r}^{\mathrm{EB}} q_{r}^{\mathrm{EB}}+w_{p}^{\mathrm{EB}} q_{p}^{\mathrm{EB}} \\
& \pi_{r}^{\mathrm{EB}}=\left(1-q_{r}^{\mathrm{EB}}-q_{s}^{\mathrm{EB}}-q_{p}^{\mathrm{EB}}-w_{r}^{\mathrm{EB}}-c_{r}\right) q_{r}^{\mathrm{EB}} \\
& \pi_{P}^{\mathrm{EB}}=\left(1-q_{r}^{\mathrm{EB}}-q_{s}^{\mathrm{EB}}-q_{p}^{\mathrm{EB}}-w_{P}^{\mathrm{EB}}-c_{p}\right) q_{P}^{\mathrm{EB}}+\left(f^{\mathrm{EB}}-c_{p}\right) q_{s}^{\mathrm{EB}}-k\left(q_{s}^{\mathrm{EB}}+q_{P}^{\mathrm{EB}}\right)^{2} .
\end{aligned}
$$

We use backward induction to solve this game. Following the timeline of Model EB in Figure 1, for any given $w_{r}^{\mathrm{EB}}, w_{p}^{\mathrm{EB}}, q_{r}^{\mathrm{EB}}$ and $f^{\mathrm{EB}}$, the supplier first chooses the direct sales to consumers $q_{s}^{\mathrm{EB}}$ to maximise its profit $\pi_{s}^{\mathrm{EB}}\left(w_{r}^{\mathrm{EB}}, w_{p}^{\mathrm{EB}}, q_{r}^{\mathrm{EB}}, f^{\mathrm{EB}}, q_{p}^{\mathrm{EB}}, q_{s}^{\mathrm{EB}}\right)$. Further, the platform owner determines the equilibrium quantities $q_{p}^{\mathrm{EB}}$ to maximise its profit $\pi_{P}^{\mathrm{EB}}\left(w_{P}^{\mathrm{EB}}, f^{\mathrm{EB}}, q_{r}^{\mathrm{EB}}, q_{P}^{\mathrm{EB}}, q_{s}^{\mathrm{EB}}\right)$ simultaneously:

$$
\begin{gathered}
q_{s}^{\mathrm{EB}}\left(w_{P}^{\mathrm{EB}}, f^{\mathrm{EB}}, q_{r}^{\mathrm{EB}}\right)=\frac{1+c_{p}-2 f^{\mathrm{EB}}+2 k-q_{r}^{\mathrm{EB}}+w_{P}^{\mathrm{EB}}-2 f^{\mathrm{EB}} k-2 k q_{r}^{\mathrm{EB}}}{2 k+3} \\
q_{p}^{\mathrm{EB}}\left(w_{P}^{\mathrm{EB}}, f^{\mathrm{EB}}, q_{r}^{\mathrm{EB}}\right)=\frac{1-2 c_{p}+f^{\mathrm{EB}}-2 k-q_{r}^{\mathrm{EB}}-2 w_{P}^{\mathrm{EB}}+2 f^{\mathrm{EB}} k+2 k q_{r}^{\mathrm{EB}}}{2 k+3} .
\end{gathered}
$$

When substituting $\quad q_{s}^{\mathrm{EB}}\left(w_{P}^{\mathrm{EB}}, f^{\mathrm{EB}}, q_{r}^{\mathrm{EB}}\right) \quad$ and $\quad q_{p}^{\mathrm{EB}}\left(w_{P}^{\mathrm{EB}}, f^{\mathrm{EB}}, q_{r}^{\mathrm{EB}}\right) \quad$ into $\quad$ equation (3.17), the platform owner determines the commission fee $f^{\mathrm{EM}}$ to maximise its profit $\pi_{P}^{\mathrm{EB}}\left(w_{P}^{\mathrm{EB}}, f^{\mathrm{EB}}, q_{r}^{\mathrm{EB}}, q_{p}^{\mathrm{EB}}\left(w_{P}^{\mathrm{EB}}, f^{\mathrm{EB}}, q_{r}^{\mathrm{EB}}\right), q_{s}^{\mathrm{EB}}\left(w_{P}^{\mathrm{EB}}, f^{\mathrm{EB}}, q_{r}^{\mathrm{EB}}\right)\right)$ :

$$
f^{\mathrm{EB}}\left(w_{P}^{\mathrm{EB}}, q_{r}^{\mathrm{EB}}\right)=\frac{5-5 q_{r}^{\mathrm{EB}}+14 k+5 c_{p}-w_{P}^{\mathrm{EB}}+4 c_{p} k-14 k q_{r}^{\mathrm{EB}}-6 k w_{P}^{\mathrm{EB}}-8 k^{2} q_{r}^{\mathrm{EB}}-4 k^{2} w_{P}^{\mathrm{EB}}+8 k^{2}}{8 k^{2}+18 k+10} .
$$

Then, when substituting $q_{s}^{\mathrm{EB}}\left(w_{P}^{\mathrm{EB}}, f^{\mathrm{EB}}, q_{r}^{\mathrm{EB}}\right), q_{p}^{\mathrm{EB}}\left(w_{P}^{\mathrm{EB}}, f^{\mathrm{EB}}, q_{r}^{\mathrm{EB}}\right)$ and $f^{\mathrm{EB}}{ }^{*}\left(w_{P}^{\mathrm{EB}}, q_{r}^{\mathrm{EB}}\right)$ into equation (3.16), the traditional retailer chooses equilibrium quantities $q_{r}^{\mathrm{EB}}$ to maximise its profit $\pi_{r}^{\mathrm{EB}}\left(w_{r}^{\mathrm{EB}}, q_{r}^{\mathrm{EB}}, q_{p}^{\mathrm{EB}}\left(w_{P}^{\mathrm{EB}}, f^{\mathrm{EB}}, q_{r}^{\mathrm{EB}}\right), q_{s}^{\mathrm{EB}}\left(w_{P}^{\mathrm{EB}}, f^{\mathrm{EB}}, q_{r}^{\mathrm{EB}}\right)\right):$

$$
\begin{aligned}
& q_{r}^{\mathrm{EB}}\left(w_{r}^{\mathrm{EB}}, w_{P}^{\mathrm{EB}}\right) \\
& =\frac{5 c_{p}-10 c_{r}+14 k+3 w_{P}^{\mathrm{EB}}-10 w_{r}^{\mathrm{EB}}+4 c_{p} k-18 c_{r} k+2 k w_{P}^{\mathrm{EB}}-18 k w_{r}^{\mathrm{EB}}-8 c_{r} k^{2}-8 k^{2} w_{r}^{\mathrm{EB}}+8 k^{2}+5}{16 k^{2}+28 k+10} .
\end{aligned}
$$

When substituting $\quad q_{s}^{\mathrm{EB}}\left(w_{P}^{\mathrm{EB}}, f^{\mathrm{EB}}, q_{r}^{\mathrm{EB}}\right), \quad q_{p}^{\mathrm{EB}}\left(w_{P}^{\mathrm{EB}}, f^{\mathrm{EB}}, q_{r}^{\mathrm{EB}}\right), \quad f^{\mathrm{EB}}\left(w_{P}^{\mathrm{EB}}, q_{r}^{\mathrm{EB}}\right) \quad$ and $q_{r}^{\mathrm{EB}}\left(w_{r}^{\mathrm{EB}}, w_{P}^{\mathrm{EB}}\right) \quad$ into equation (3.15), the supplier determines the wholesale price $w_{r}^{\mathrm{EB}}$ 
and $w_{p}^{\mathrm{EB}}$ for the traditional retailer and platform owner to maximise its profit $\pi_{s}^{\mathrm{EB}}\left(w_{r}^{\mathrm{EB}}, w_{p}^{\mathrm{EB}}, q_{r}^{\mathrm{EB}}{ }^{*}\left(w_{r}^{\mathrm{EB}}, w_{P}^{\mathrm{EB}}\right), f^{\mathrm{EB}}{ }^{*}\left(w_{P}^{\mathrm{EB}}, q_{r}^{\mathrm{EB}}\right), q_{p}^{\mathrm{EB}}\left(w_{P}^{\mathrm{EB}}, f^{\mathrm{EB}}, q_{r}^{\mathrm{EB}}\right), q_{s}^{\mathrm{EB}}\left(w_{P}^{\mathrm{EB}}, f^{\mathrm{EB}}, q_{r}^{\mathrm{EB}}\right)\right) . \quad$ Then, $\quad$ we can obtain the equilibrium wholesale prices, commission fees, demands and profits, as summarised in Lemma 3.4.

\section{Lemma 3.4. Under Model EB:}

(1) The equilibrium wholesale prices, quantities, and commission fee are:

$$
\begin{aligned}
w_{r}^{\mathrm{EB}}= & \frac{\left[\begin{array}{l}
9 c_{p}-98 c_{r}+358 k+56 c_{p} k-414 c_{r} k+80 c_{p} k^{2}+32 c_{p} k^{3}-568 c_{r} k^{2} \\
-320 c_{r} k^{3}-64 c_{r} k^{4}+488 k^{2}+288 k^{3}+64 k^{4}+89
\end{array}\right]}{4\left(32 k^{4}+160 k^{3}+287 k^{2}+214 k+53\right)} \\
w_{p}^{\mathrm{EB}} & =\frac{\left[\begin{array}{l}
10 c_{r}-55 c_{p}+161 k-189 c_{p} k+28 c_{r} k-196 c_{p} k^{2} \\
-64 c_{p} k^{3}+26 c_{r} k^{2}+8 c_{r} k^{3}+170 k^{2}+56 k^{3}+45
\end{array}\right]}{2\left(32 k^{4}+160 k^{3}+287 k^{2}+214 k+53\right)} \\
q_{r}^{\mathrm{EB}} & =\frac{\left[\begin{array}{l}
32 c_{p}-54 c_{r}+99 k+63 c_{p} k-162 c_{r} k+40 c_{p} k^{2}+8 c_{p} k^{3} \\
-180 c_{r} k^{2}-88 c_{r} k^{3}-16 c_{r} k^{4}+140 k^{2}+80 k^{3}+16 k^{4}+22
\end{array}\right]}{2\left(32 k^{4}+287 k^{2}+160 k^{3}+53+214 k\right)} \\
f^{\mathrm{EB}} & =\frac{\left[\begin{array}{l}
85 c_{p}+52 c_{r}+418 k+396 c_{p} k+254 c_{r} k+642 c_{p} k^{2}+444 c_{p} k^{3}+112 c_{p} k^{4} \\
\left.+470 c_{r} k^{2}+420 c_{r} k^{3}+184 c_{r} k^{4}+32 c_{r} k^{5}+892 k^{2}+924 k^{3}+472 k^{4}+96 k^{5}+747 k^{3}+501 k^{2}+267 k+53\right)
\end{array}\right]}{32 k^{4}+160 k^{3}+287 k^{2}+214 k+53} . \\
q_{s}^{\mathrm{EB}}= & \frac{(k+1)\left(2 c_{r}-11 c_{p}+25 k-29 c_{p} k+4 c_{r} k-16 c_{p} k^{2}+2 c_{r} k^{2}+14 k^{2}+9\right)}{4\left(32 k^{5}+192 k^{4}+447 k^{3}+501 k^{2}+267 k+53\right)} \\
q_{P}^{\mathrm{EB}}= & \frac{\left[\begin{array}{l}
40 c_{r}-61 c_{p}+64 k-178 c_{p} k+114 c_{r} k-168 c_{p} k^{2}-52 c_{p} k^{3} \\
+118 c_{r} k^{2}+52 c_{r} k^{3}+8 c_{r} k^{4}+50 k^{2}-8 k^{4}+21
\end{array}\right]}{4(36)}
\end{aligned}
$$

(2) The equilibrium profits of the supplier, traditional retailer, and platform owner are:

$$
\pi_{s}^{\mathrm{EB}^{*}}=\frac{\left[\begin{array}{l}
72 c_{p}^{2} k^{3}+236 c_{p}^{2} k^{2}+252 c_{p}^{2} k+87 c_{p}^{2}-32 c_{p} c_{r} k^{4}-192 c_{p} c_{r} k^{3}-412 c_{p} c_{r} k^{2} \\
-380 c_{p} c_{r} k-128 c_{p} c_{r}+32 c_{p} k^{4}+48 c_{p} k^{3}-60 c_{p} k^{2}-124 c_{p} k-46 c_{p}+32 c_{r}^{2} k^{5} \\
+208 c_{r}^{2} k^{4}+536 c_{r}^{2} k^{3}+684 c_{r}^{2} k^{2}+432 c_{r}^{2} k+108 c_{r}^{2}-64 c_{r} k^{5}-384 c_{r} k^{4}-880 c_{r} k^{3} \\
-956 c_{r} k^{2}-484 c_{r} k-88 c_{r}+32 k^{5}+176 k^{4}+416 k^{3}+508 k^{2}+304 k+67
\end{array}\right]}{8(k+1)\left(32 k^{4}+287 k^{2}+160 k^{3}+53+214 k\right)},
$$




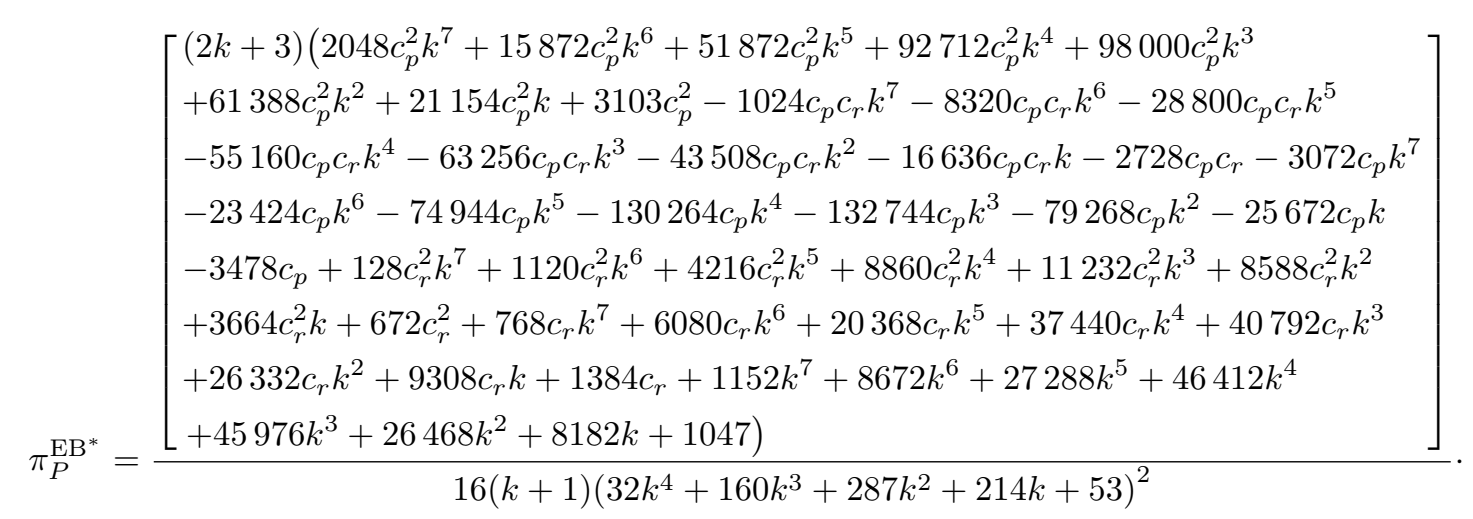

\section{Analysis}

\subsection{Model N vs. Model EM}

Comparing the outcomes of Model $\mathrm{N}$ and EM, we can address the question posed at the beginning of this paper: When the platform owner forgoes its entry option, what implications does supplier encroachment have for the supplier and traditional retailer? In particular, we find that the online platform's selling cost plays a critical role in shaping both parties' profitability. That is,

Proposition 4.1. (i) Supplier encroachment with an online platform increases the supplier's profit if $c_{p}<c_{p 1}$ or $c_{p}>c_{p 2}$ (i.e., $\left.\pi_{s}^{\mathrm{EM}^{*}}>\pi_{s}^{\mathrm{N}^{*}}\right)$, and also increases the traditional retailer's profit if $c_{p}>c_{p 3}$ (i.e., $\pi_{r}^{\mathrm{EM}^{*}}>$ $\left.\pi_{r}^{\mathrm{N}^{*}}\right)$.

(ii) There is a "win-win" outcome if $c_{p}>c_{p 3}$ (i.e., $\left.\pi_{s}^{\mathrm{EM}^{*}}>\pi_{s}^{\mathrm{N}^{*}}, \pi_{r}^{\mathrm{EM}^{*}}>\pi_{r}^{\mathrm{N}^{*}}\right)$, a "win-lose" outcome if $c_{p}<c_{p 1}$ or $c_{p 2}<c_{p}<c_{p 3}$ (i.e., $\pi_{s}^{\mathrm{EM}^{*}}>\pi_{s}^{\mathrm{N}^{*}}, \pi_{r}^{\mathrm{EM}^{*}}<\pi_{r}^{\mathrm{N}^{*}}$ ) and a "lose-lose" outcome if $c_{p 1}<c_{p}<c_{p 2}$ (i.e., $\pi_{s}^{\mathrm{EM}^{*}}<\pi_{s}^{\mathrm{N}^{*}}, \pi_{r}^{\mathrm{EM}^{*}}<\pi_{r}^{\mathrm{N}^{*}}$ ), for the supplier and traditional retailer.

As Figure 3a shows, Proposition 4.1(i) reveals that when the online platform's selling cost is relatively small or large, supplier encroachment is always beneficial for the supplier. Note that in Model EM, the supplier's profits in this setting come from two sources: selling products through the online platform and wholesaling products to the traditional retailer. When the online platform's selling cost decreases (i.e., $c_{p}<c_{p 1}$ ), the online platform will charge a lower commission fee (notice from Lem. 3.1 that $\partial f^{\mathrm{EM}^{*}} / \partial c_{p}>0$ ). As a result, the supplier can gain a higher marginal profit per unit through the online platform. Thus, to gain more profits when $c_{p}<c_{p 1}$, the supplier gives less consideration to the traditional retailer's revenue and encroaches heavily on the retail market. Moreover, when $c_{p}>c_{p 2}$, the major decision faced by the supplier is similar to that explored in Arya et al. [3]. In the traditional retail channel, the supplier usually sets a lower wholesale price to avoid unduly reducing the traditional retailer's demand (notice that $w_{r}^{\mathrm{EM}^{*}}$ is less than $w_{r}^{\mathrm{N}^{*}}$ ). As a result, although the platform owner will charge a relatively high fee to the supplier when $c_{p}>c_{p 2}$, the supplier can benefit more from the traditional retailer by lowering the wholesale price.

As Figure 3b shows, Proposition 4.1(i) further indicates that in the scenario where the platform forgoes its entry option, supplier encroachment may benefit or hurt the traditional retailer depending on the change in the online platform's selling costs. Specifically, when the online platform's selling cost is sufficiently high (i.e., $c_{p}>c_{p 3}$ ), there is a higher commission charged by the online platform to the supplier, and wholesaling products through the traditional channel is more profitable than selling products through the online platform. Thus, although the supplier encroaches on the traditional retailer's market, she cares greatly about the traditional retailer's profitability and sets a relatively lower wholesale price for the traditional retailer, leading to an increase in the traditional retailer's profitability from the lower wholesale price and outweighing the loss from the supplier encroachment. Then, as illustrated in Figure 3c, Proposition 4.1(ii), a "win-win" outcome arises when $c_{p}>c_{p 3}$. Conversely, when $c_{p}<c_{p 3}$, the latter component dominates, which leads to "win-lose" or "lose-lose" outcomes for the supplier and the traditional retailer. 


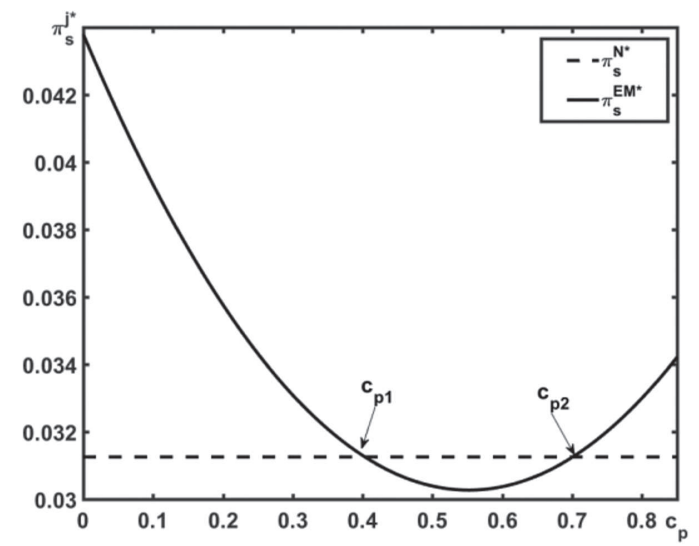

(a)

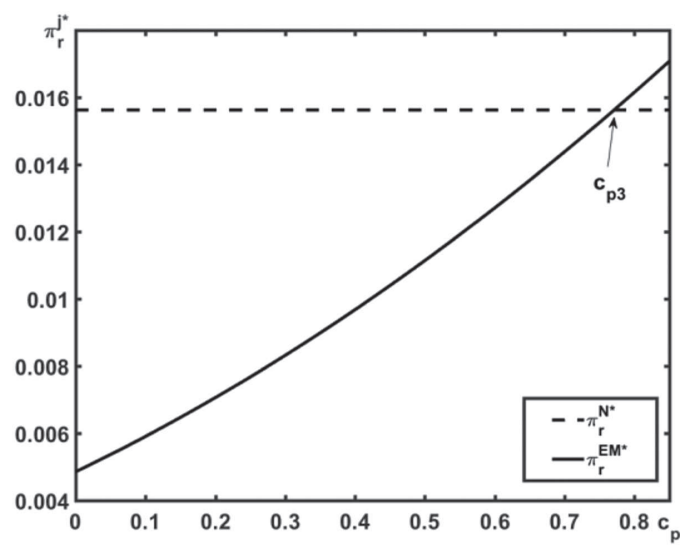

(b)

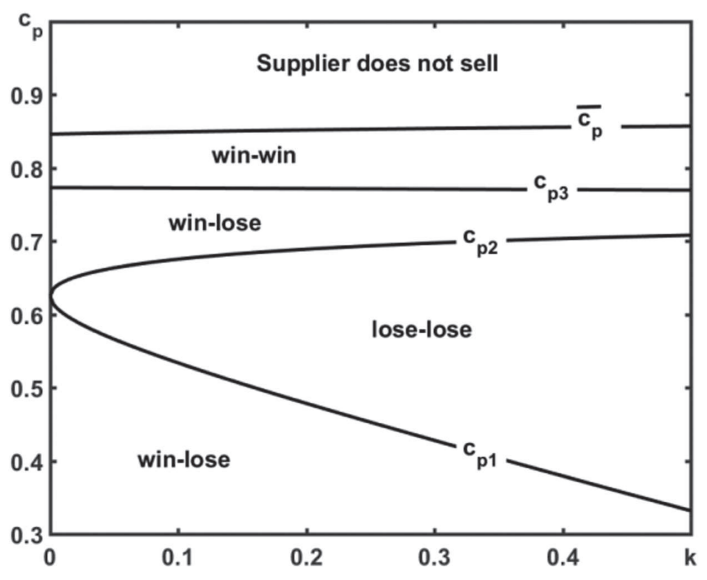

(c)

Figure 3. The possible outcomes in numerical experiments. (a) Variations of $\pi_{s}^{\mathrm{EM}^{*}}$ and $\pi_{s}^{\mathrm{N}^{*}}$. (b) Variations of $\pi_{r}^{\mathrm{EM}}$ and $\pi_{r}^{\mathrm{N}^{*}}$. (c) The possible outcomes between Model EM and Model N.

In particular, the "lose-lose" outcome would arise when the online platform's selling cost is intermediate (i.e., $c_{p 1}<c_{p}<c_{p 2}$ ), that is, when the retail cost disadvantage of online channels is not profound. The supplier shows no preference between the channels because the marginal profits per unit are similar between wholesaling products in the traditional channel and selling products through the online platform. Consequently, both partners have a close game between both channels. On the one hand, anticipating that the marginal profits per unit for the supplier are similar between the channels, the traditional retailer will vigorously protect his profits if the supplier encroaches into the retail market with an online platform. On the other hand, to deal with the competition from the traditional retailer and the commission fee charged by the platform owner, the supplier must make herself unprofitable by encroaching on the retail market by selling products through the online platform, which leads to a "lose-lose" outcome.

Note, in this example, $c_{r}=0.5, k=0.7$. 


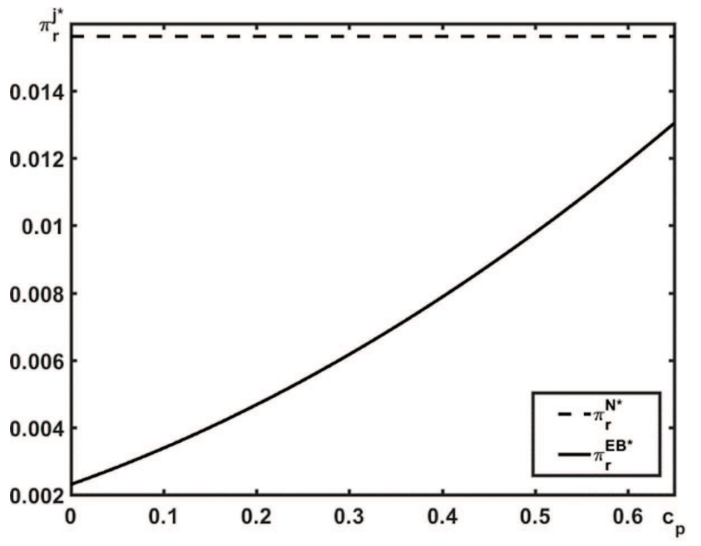

(a)

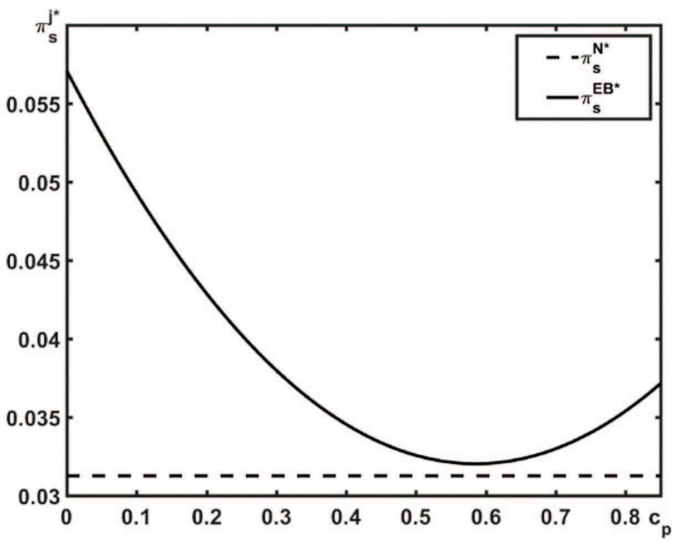

(b)

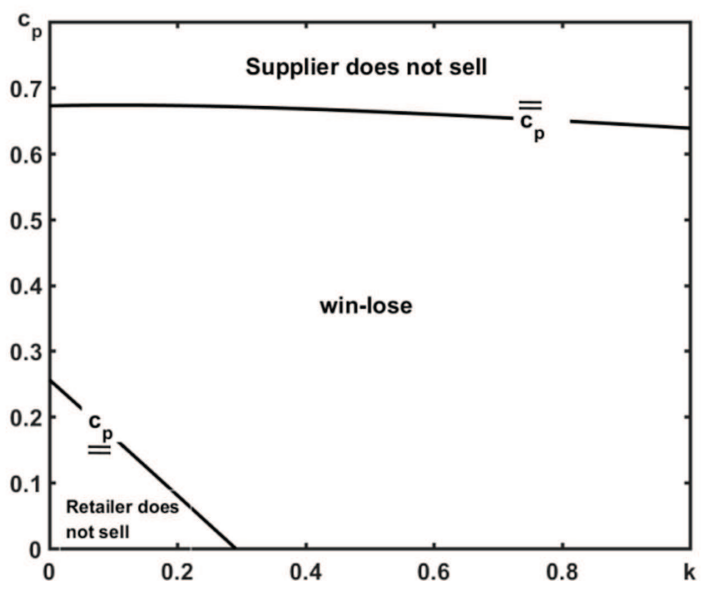

(c)

FigurE 4. The possible outcomes in numerical experiments. (a) Variations of $\pi_{r}^{\mathrm{EB}^{*}}$ and $\pi_{r}^{\mathrm{N}^{*}}$. (b) Variations of $\pi_{s}^{\mathrm{EB}}$ and $\pi_{s}^{\mathrm{N}^{*}}$. (c) The possible outcomes between Model EB and Model N.

\subsection{Model N vs. Model EB}

As mentioned earlier, the primary interest of this study is to understand how supplier encroachment with an online platform affects all parties' profitability. Exploring the rationale behind this goal, we now compare the final net change in the supplier's and traditional retailer's profitability from Model N to Model EB. The difference here is that the supplier has encroached on the retail market with an online platform and the platform owner retains its encroachment option. From this perspective, we can conclude our findings in the following proposition:

Proposition 4.2. (i) Compared to Model N, when the platform retains its encroachment option, supplier encroachment is always beneficial for the supplier but detrimental for the traditional retailer (i.e., $\pi_{s}^{\mathrm{EB}^{*}}>$ $\left.\pi_{s}^{\mathrm{N}^{*}} \pi_{r}^{\mathrm{EB}}<\pi_{r}^{\mathrm{N}^{*}}\right)$, and

(ii) There are only "win-lose" outcomes for the supplier and traditional retailer. 
Intuitively, in Model EB, the flexibility of wholesaling products to an online platform allows the supplier to rely less on the traditional retailer, which makes the traditional retailer less likely to benefit from the encroachment; however, Proposition 4.2 shows a stronger result, where the traditional retailer no longer wins. Figure $4 \mathrm{a}$ illustrates this result. Before explaining this, it is necessary to examine supplier's profits, which in this setting, come from three sources: selling products through the online platform, wholesaling products to the traditional retailer and wholesaling products to the platform owner. It was found that if the platform retains its encroachment option, the supplier would be better off in Model EB than in Model N, as shown in Figure 4b. In particular, there could be a possible alliance between the supplier and platform owner when the traditional retailer's cost advantage is sufficiently profound (i.e., in exchange for a lower commission fee charged by the platform owner, the supplier would offer a lower wholesale price to the platform owner compared to the traditional retailer). Thus, when the platform retains its encroachment option, the retailer in the traditional channel is in a more challenging position than that confronting a supplier-owned channel: he is not only competing with the products from the supplier but is also dealing with the products that are resold by the online retail platform. Hence, the traditional retailer is always worse off when the platform retains its encroachment option. Put differently, as illustrated in Figure 4c, there are only "win-lose" outcomes for the supplier and the traditional retailer under this scenario.

Note, in this example, $c_{r}=0.5, k=0.7$.

\subsection{Model ET vs. Model EB}

Now, we answer the following question: When the platform owner retains its entry option, what implications does supplier encroachment have for the supplier, traditional retailer and platform owner? We compare the supplier, traditional retailer and platform owner's profitability in Model EB to their profitability in Model ET. The difference is that the platform retains its encroachment option, and the supplier decides whether they will encroach on the retail market by selling products directly. Then, we have the following proposition:

Proposition 4.3. (i) Compared to Model ET, under the scenario of Model EB, supplier encroachment is always beneficial for the supplier $\left(\pi_{s}^{\mathrm{EB}}>\pi_{s}^{\mathrm{ET}}\right)$ but detrimental for the traditional retailer (i.e., $\pi_{r}^{\mathrm{EB}^{*}}<$ $\left.\pi_{r}^{\mathrm{ET}}\right)$.

(ii) Compared to Model ET, the platform owner would be better off (i.e., $\pi_{P}^{\mathrm{EB}^{*}}>\pi_{P}^{\mathrm{EM}^{*}}$ ) under the scenario of Model EB.

Proposition 5.1(i) demonstrates that the profits of the supplier are higher when the platform retains its encroachment option, but the traditional retailer's profit is lower in Model EB than in Model ET. As mentioned earlier, in Model EB, supplier encroachment creates an additional distribution channel for the supplier, leading to greater profit for itself. However, the retailer in the traditional channel is in a more challenging position than the supplier; they are not only competing with products resold by the platform owner but may have to deal with products from the supplier. The additional units offered by the supplier through the online platform increase the market competition. Such increased competition reduces the quantities of products in the traditional channel.

Proposition 5.1(ii) further reveals that the platform owner may be pleased to see the supplier encroaching on the retailers' market through its online platform. The reason is that the platform owner's profitability comes from two sources in Model EB: charging a commission fee to the supplier and selling products directly. Although supplier encroachment leads to additional competition from the supplier, who distributes products through the online platform, the platform owner benefits from the flexibility of strategically charging a commission fee to the supplier to limit additional competition. Particularly, more quantities of the product can be sold through the online platform in Model EB than in Model ET, increasing the platform owner's profits. This result explains why more retail platforms, such as Amazon and JD.com, tend to introduce a marketplace that allows the supplier to sell products directly for a commission fee. 


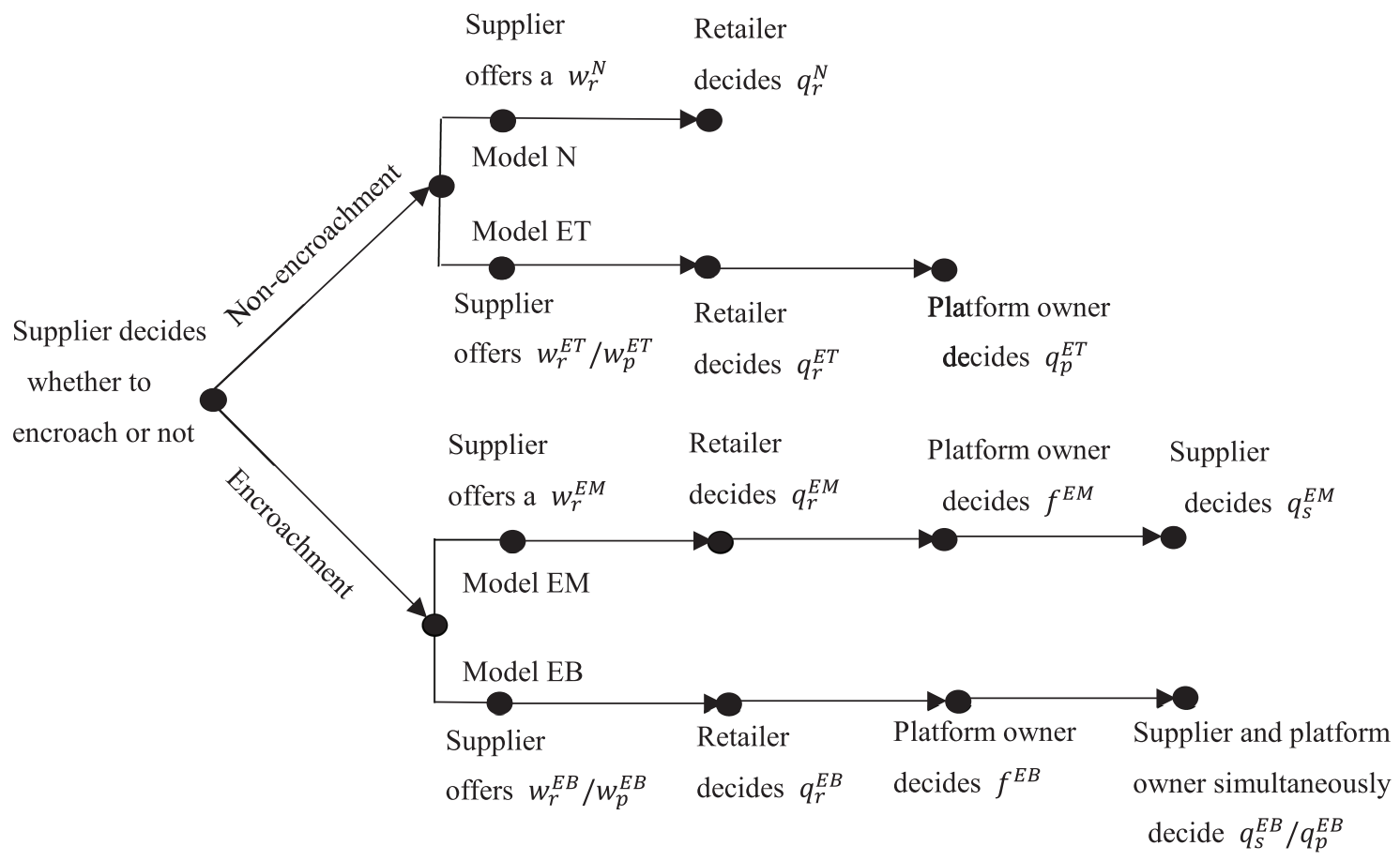

FiguRE 5. Timeline in the simultaneous setting.

\section{EXTENSION}

The analysis in Section 4 is based on the sequential setting in which the supplier and the platform owner choose their sales quantities after the traditional retailer chooses its sales quantities. In this section, as summarised in Figure 5, we now consider simultaneous encroachment settings to further assess the robustness of our key findings ${ }^{4}$.

As in the prior section, we can adopt backward induction to obtain the equilibrium solutions in Model EM, Model ET and Model EB under the simultaneous setting ${ }^{5}$.

As before, we first address the changes in the profits of the supplier and traditional retailer from Model $\mathrm{N}$ to Model EM under the simultaneous setting. We provide the following conclusion:

Proposition 5.1. Under the simultaneous setting:

There is still a "win-lose" outcome if $c_{p}<c_{p 4}\left(\right.$ i.e., $\left.\pi_{s}^{\mathrm{EM}^{*}}>\pi_{s}^{\mathrm{N}^{*}}, \pi_{r}^{\mathrm{EM}^{*}}<\pi_{r}^{\mathrm{N}^{*}}\right)$ and a "lose-lose" outcome if $c_{p 4}<c_{p}<c_{p 5}$ (i.e., $\pi_{s}^{\mathrm{EM}^{*}}<\pi_{s}^{\mathrm{N}^{*}}, \pi_{r}^{\mathrm{EM}^{*}}<\pi_{r}^{\mathrm{N}^{*}}$ ) for the supplier and traditional retailer. While a new "lose-win" outcome arises if $c_{p 5}<c_{p}$ (i.e., $\pi_{s}^{\mathrm{EM}^{*}}<\pi_{s}^{\mathrm{N}^{*}}$ and $\pi_{r}^{\mathrm{EM}^{*}}>\pi_{r}^{\mathrm{N}^{*}}$ ), and the "win-win" outcome disappears.

First, compared to the sequential setting, Proposition 5.1 reveals that the simultaneous decision would have the thresholds $c_{p 4}<c_{p 1}<c_{p 2}$ and $c_{p 5}<c_{p 3}$ (see Fig. 6). That means the simultaneous decision reduces the supplier's incentive to encroach with an online platform due to its profitable range becoming smaller.

Proposition 5.1 further reveals that when the online platform's sales cost is low, the results in Proposition 4.1 are quite robust; they are labelled as the "win-lose" and "lose-lose" outcomes in Figure 6. Furthermore, unlike Proposition 4.1, Proposition 5.1 indicates that a "lose-win" outcome arises yet a "win-win" outcome no longer

\footnotetext{
${ }^{4}$ We thank an anonymous reviewer for pointing out such constructive suggestion.

${ }^{5}$ For brevity, all lemmas and their detailed proofs are provided in the Appendix A.
} 


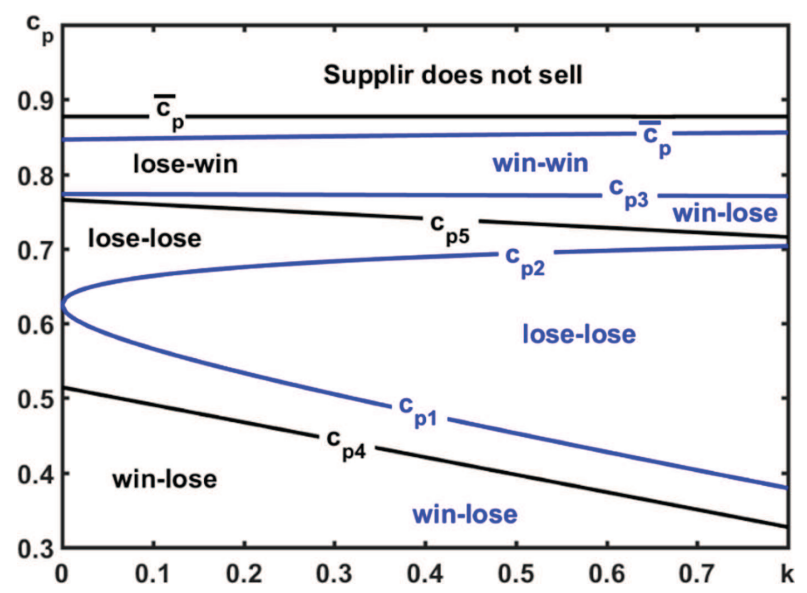

Figure 6. The possible outcomes between Model EM and Model N under both settings (The outcomes under the sequential setting are shown in blue and the outcomes under the simultaneous setting are shown in black).

exists when the online platform's sales cost is relatively large $\left(i . e ., c_{p 5}<c_{p}\right)$ in the simultaneous setting. This can be interpreted as follows. When $c_{p 5}<c_{p}$, the supplier lowers wholesale prices to the traditional retailer more substantially in the simultaneous setting than in the sequential setting. The lower wholesale price results in a direct loss for the supplier from the traditional channel. By contrast, the lower wholesale price compensates the traditional retailer's loss due to the supplier encroachment. As such, if $c_{p 5}<c_{p}$, compared to the sequential setting, the supplier can be worse off; the traditional retailer is better off in Model EM than Model N under simultaneous setting, which results in the appearance of a "lose-win" outcome and the disappearance of the "win-win" outcome.

Note, in this example, $c_{r}=0.5$.

Next, comparing the outcomes in Model EB and Model N, we can obtain the supplier and the traditional retailer' s profitability, with the platform owner retaining its entry option in the simultaneous setting, and provide the following conclusion:

Proposition 5.2. Under the simultaneous setting:

Besides a "win-lose" outcome if $c_{p}<c_{p 6}$ (i.e., $\pi_{s}^{\mathrm{EB}^{*}}>\pi_{s}^{\mathrm{N}^{*}}, \pi_{r}^{\mathrm{EB}^{*}}<\pi_{r}^{\mathrm{N}^{*}}$ ), a new "lose-lose" outcome arises if $c_{p}>c_{p 6}$ (i.e., $\pi_{s}^{\mathrm{EB}^{*}}<\pi_{s}^{\mathrm{N}^{*}}, \pi_{r}^{\mathrm{EB}}<\pi_{r}^{\mathrm{N}^{*}}$ ) for the supplier and traditional retailer.

Consistent with Proposition 4.2, Proposition 5.2 indicates that Model EB would always be detrimental to the traditional retailer when compared to Model N. This result is quite robust and the intuition behind it is not repeated here. Notably, Proposition 4.2 also shows that when the platform retains its entry option, the supplier encroachment is always beneficial for the supplier in the sequential setting.

However, Proposition 5.2 reveals that the supplier's encroachment would hurt her when the online platform's sales cost in the simultaneous setting is relatively large (i.e., $c_{p}>c_{p 6}$ ). This reveals that - in contrast to Proposition 4.2, under the setting of simultaneous decisions - besides the "win-lose" outcome, there is the additional result of "lose-lose" for the supplier and the traditional retailer (see Fig. 7). This result can be interpreted as follows. First, in Model EB, fewer products are sold through the traditional channel under the simultaneous setting than the sequential setting. Second, in Model EB, when $c_{p}>c_{p 6}$, the supplier may charge a lower wholesale price to traditional retailers under the simultaneous setting than the sequential setting. Thus, when $c_{p}>c_{p 6}$, compared to the sequential setting, the supplier is worse off in Model EB than in Model N under the simultaneous setting. 


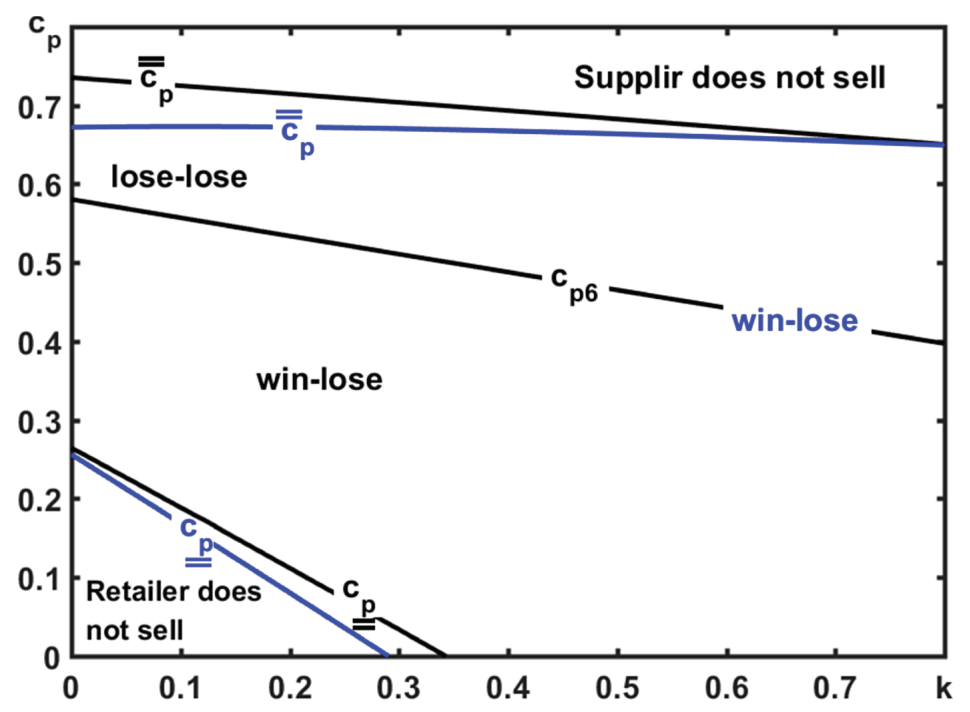

Figure 7. The possible outcomes between Model EB and Model N under both settings (The outcomes under the sequential setting are shown in blue and the outcomes under the simultaneous setting are shown in black).

Note, in this example, $c_{r}=0.5$.

Finally, we compare the profits of the supplier, traditional retailer and platform owner from Model ET to Model EB under the simultaneous settings. We provide the following conclusion.

Proposition 5.3. Under the simultaneous setting:

Compared to Model ET, under Model EB, supplier encroachment still increases the supplier and platform owners' profits but decreases the traditional retailer's profit.

Consistent with Proposition 4.3, Proposition 5.3 shows that when the platform owner retains its entry option to resell the products directly in the simultaneous setting, supplier encroachment would always be beneficial to the supplier and platform owner but detrimental to the traditional retailer. That means timing patterns do not affect the supplier's preference to encroach on the retailer's market, with the platform owner retaining its entry option. This result is quite robust.

\section{Conclusion}

E-commerce provides suppliers with the potential flexibility to operate an online arm with an online platform in addition to their physical stores. For example, in the electronics industry, many brand-name suppliers, including Apple, IBM, and Lenovo, have sold their products online on Amazon as well as through stores like Best Buy and Circuit City. Although such supplier encroachment is becoming increasingly prevalent in ecommerce markets, the extant studies about supplier encroachment traditionally assume that the supplier sells products directly to consumers through its e-channel and ignores the roles played by online platforms in retail channels.

This research complements this stream of research by investigating the implications of supplier encroachment with an online platform under two scenarios. In the first scenario, the supplier determines whether to encroach on the retail market through an online platform when the platform owner forgoes its option to resell the products from the supplier (Model N and Model EM). In the second scenario, the supplier chooses whether to encroach on the retail market through an online platform when the platform owner retains their entry option (Model ET 
and Model EB). In four models, we do not facilitate product differentiation or price discrimination ${ }^{6}$. This is to reflect the fact that suppliers are concerned about reputational backlash when customers discover different pricing schemes for different channels, as is noted in Cattani et al. [7] and Arya et al. [3]. For example, Apple charges the same prices for its Mac, iPhone, and iPad on Amazon as that in retail stores. Particularly, our models extend the work of Cattani et al. [7] and Arya et al. [3], in which the supplier encroaches on retail market with its own direct channel, to highlight the strategic response to supplier encroachment when the platform owner has the flexibility to forgo and/or retain entry options.

A central result obtained is that, unlike a supplier-owned direct channel and in addition to "win-win" outcomes for the supplier and traditional retailer, supplier encroachment with an online platform may also lead to "win-lose" and "lose-lose" outcomes. When the platform owner retains its entry option, such encroachment is always detrimental for the traditional retailer but beneficial for the supplier. And the platform owner would be better off. Extending all models to the case of both parties making decisions simultaneously, we further find that the simultaneous decision reduces the supplier's incentive to encroach with an online platform but mitigates the traditional retailer's loss from the supplier encroachment.

The model proposed in this study has some limitations as well. First, in all the models, asymmetric information settings have not been addressed. The analysis and insights may be fundamentally different, as revealed in Li et al. [23,24]. Second, the supplier is viewed as the Stackelberg leader; that is, she considers the profit-maximising actions of the traditional retailer and simultaneously sets the wholesale price. However, when confronting a dominant traditional retailer, such as Home Depot, the power structure may change. Certainly, it is possible that pricing decisions are made by the traditional retailer and, as a result, the negotiated pricing may be more appropriate in practice. Third, the model assumes that the traditional retailer is a bricks-and-mortar reseller - an assumption that, although common in the literature of e-commerce $[3,17,38]$, does not reflect the reality that many traditional retailers have ventured into the online world. Finally, to avoid unnecessary complications, this paper does not focus on the supplier own direct channel, however, in reality, many brand-name suppliers, including Apple, IBM and Lenovo, have opened their own direct channels. This opens up the potential for more realistic models of supplier encroachment.

\section{Appendix A.}

Proof of Lemma 3.1. From the equation $p=1-Q$, it can be found that the demand with the no-encroachment setting is $p=1-q_{r}^{\mathrm{N}}$. Based on the timeline in the Model $\mathrm{N}$ under sequential setting, the equilibrium decisions are solved by backward induction. That is, given wholesale price $w_{r}^{\mathrm{N}}$, the traditional retailer's problem is $\max _{q_{r}^{\mathrm{N}}} \pi_{r}^{\mathrm{N}}=\left(1-q_{r}^{\mathrm{N}}-w_{r}^{\mathrm{N}}-c_{r}\right) q_{r}^{\mathrm{N}}$. By applying the first-order condition to $\pi_{r}^{\mathrm{N}}$ with respect to $q_{r}^{\mathrm{N}}$, we can obtain $q_{r}^{\mathrm{N}^{*}}=\frac{1-w_{r}^{\mathrm{N}}-c_{r}}{2}$. Anticipating the traditional retailer's response, $q_{r}^{\mathrm{N}^{*}}\left(w_{r}^{\mathrm{N}}\right)$, the supplier chooses optimal wholesale price to maximise its profit. In other words, $\max _{w_{r}^{\mathrm{N}}} \pi_{s}^{\mathrm{N}}=w_{r}^{\mathrm{N}}\left(\frac{1-w_{r}^{\mathrm{N}}-c_{r}}{2}\right)$, and this expression yields $w_{r}^{\mathrm{N}^{*}}=\frac{1-c_{r}}{2}$. Hence, the equilibrium wholesale quantities without encroachment are $q_{r}^{\mathrm{N}^{*}}=\frac{1-c_{r}}{4}$, and the equilibrium profits are $\pi_{s}^{\mathrm{N}^{*}}=\frac{\left(1-c_{r}\right)^{2}}{8}$ and $\pi_{r}^{\mathrm{N}^{*}}=\frac{\left(1-c_{r}\right)^{2}}{16}$.

Proof of Lemma 3.2. Based on the timeline in the Model EM under sequential setting, solving the first-order condition of the supplier's profit from equation (3.4) with respect to $q_{s}^{\mathrm{EM}}$ yields $q_{s}^{\mathrm{EM}^{*}}=$ $\frac{1-q_{r}^{\mathrm{EM}}-f^{\mathrm{EM}}}{2}$. After substituting $q_{s}^{\mathrm{EM}^{*}}\left(q_{r}^{\mathrm{EM}}, f^{\mathrm{EM}}\right)$ into equation (3.6), the platform owner's profit is given: $\pi_{p}^{\mathrm{EM}^{*}}=\left(1-q_{r}^{\mathrm{EM}}-f^{\mathrm{EM}}\right)\left(2 f^{\mathrm{EM}}-2 c_{p}-k+f^{\mathrm{EM}} k+k q_{r}^{\mathrm{EM}}\right) / 4$, by applying the first-order condition to it with respect to $f^{\mathrm{EM}}$, we can obtain $f^{\mathrm{EM}^{*}}=\frac{c_{p}+k-q_{r}^{\mathrm{EM}}-k q_{r}^{\mathrm{EM}}+1}{k+2}$. Plugging $q_{s}^{\mathrm{EM}}\left(q_{r}^{\mathrm{EM}}, f^{\mathrm{EM}}\right)$ and $f^{\mathrm{EM}^{*}}\left(q_{r}^{\mathrm{EM}}\right)$ into the traditional retailer's profit, the problem of the traditional retailer is given: $\pi_{r}^{\mathrm{EM}^{*}}=$

\footnotetext{
${ }^{6}$ Liu et al. [26], Ofek et al. [30] and Yoon et al. [41] also provide strong support for the assumption that prices are consistent between different channels.
} 
$\frac{q_{r}^{\mathrm{EM}}\left(-4 c_{r}+c_{p}+2 k-3 q_{r}^{\mathrm{EM}}-4 w_{r}^{\mathrm{EM}}-2 c_{r} k-2 k q_{r}^{\mathrm{EM}}-2 k w_{r}^{\mathrm{EM}}+3\right)}{2(k+2)}$. Solving first-order condition of the traditional retailer' profit yields $q_{r}^{\mathrm{EM}^{*}}=\frac{3+c_{p}-4 c_{r}+2 k-2 c_{r} k-4 w_{r}^{\mathrm{EM}}-2 k w_{r}^{\mathrm{EM}}}{2(3+2 k)}$.

Plugging $q_{r}^{\mathrm{EM}^{*}}\left(w_{r}^{\mathrm{EM}}\right), f^{\mathrm{EM}^{*}}\left(q_{r}^{\mathrm{EM}}\right)$ and $q_{s}^{\mathrm{EM}^{*}}\left(q_{r}^{\mathrm{EM}}, f^{\mathrm{EM}}\right)$ into the supplier's profit, we have:

$$
\begin{aligned}
\pi_{s}^{\mathrm{EM}^{*}}= & \frac{\left(4 c_{r}-7 c_{p}+2 k+4 w_{r}^{\mathrm{EM}}-4 c_{p} k+2 c_{r} k+2 k w_{r}^{\mathrm{EM}}+3\right)^{2}}{16\left(2 k^{2}+7 k+6\right)^{2}} \\
& \times \frac{w_{r}^{\mathrm{EM}}\left(4 c_{r}-c_{p}-2 k+4 w_{r}^{\mathrm{EM}}+2 c_{r} k+2 k w_{r}^{\mathrm{EM}}-3\right)}{4 k+6} .
\end{aligned}
$$

Therefore, we can derive the optimal $w_{r}^{\mathrm{EM}}$ based on the first-order condition. That is

$$
w_{r}^{\mathrm{EM}^{*}}=\frac{5 c_{p}-44 c_{r}+68 k+10 c_{p} k-78 c_{r} k+4 c_{p} k^{2}-44 c_{r} k^{2}-8 c_{r} k^{3}+40 k^{2}+8 k^{3}+39}{16 k^{3}+88 k^{2}+158 k+92} .
$$

Furthermore, substituting the optimal wholesale prices $w_{r}^{\mathrm{EM}^{*}}$ into $q_{r}^{\mathrm{EM}^{*}}\left(w_{r}^{\mathrm{EM}}\right), f^{\mathrm{EM}}{ }^{*}\left(q_{r}^{\mathrm{EM}}\right)$ and $q_{s}^{\mathrm{EM}^{*}}\left(q_{r}^{\mathrm{EM}}, f^{\mathrm{EM}}\right)$, we can derive the optimal quantities and commission fee. That is,

$$
\begin{aligned}
q_{r}^{\mathrm{EM}^{*}} & =\frac{3 c_{p}-8 c_{r}+7 k+c_{p} k-8 c_{p} k-2 c_{r} k^{2}+2 k^{2}+5}{8 k^{2}+28 k+23}, \\
f^{\mathrm{EM}} & =\frac{10 c_{p}+4 c_{r}+15 k+7 c_{p} k+6 c_{r} k+2 c_{r} k^{2}+6 k^{2}+9}{8 k^{2}+28 k+23} \quad \text { and } \\
q_{s}^{\mathrm{EM}^{*}} & =\frac{4 c_{r}-13 c_{p}+6 k-8 c_{p} k+2 c_{r} k+9}{2\left(8 k^{2}+28 k+23\right)} .
\end{aligned}
$$

Substituting the optimal $w_{r}^{\mathrm{EM}^{*}}, q_{r}^{\mathrm{EM}^{*}}, f^{\mathrm{EM}^{*}}$ and $q_{s}^{\mathrm{EM}^{*}}$ into equations (3.4), (3.5) and (3.6), we have the profits of all players as follow:

$$
\begin{aligned}
& \pi_{s}^{\mathrm{EM}^{*}}=\frac{\left[\begin{array}{l}
9 c_{p}^{2} k+16 c_{p}^{2}-4 c_{p} c_{r} k^{2}-20 c_{p} c_{r} k-24 c_{p} c_{r}+4 c_{p} k^{2}+2 c_{p} k-8 c_{p}+4 c_{r}^{2} k^{3}+24 c_{r}^{2} k^{2} \\
+48 c_{r}^{2} k+32 c_{r}^{2}-8 c_{r} k^{3}-44 c_{r} k^{2}-76 c_{r} k-40 c_{r}+4 k^{3}+20 k^{2}+37 k+24
\end{array}\right]}{4\left(8 k^{3}+44 k^{2}+79 k+46\right)} \\
& \pi_{p}^{\mathrm{EM}^{*}}=\frac{(k+2)\left(4 c_{r}-13 c_{p}+6 k-8 c_{p} k+2 c_{r} k+9\right)^{2}}{4\left(8 k^{2}+28 k+23\right)^{2}} \text { and } \\
& \pi_{r}^{\mathrm{EM}^{*}}=\frac{(2 k+3)\left(3 c_{p}-8 c_{r}+7 k+c_{p} k-8 c_{r} k-2 c_{r} k^{2}+2 k^{2}+5\right)^{2}}{2\left(8 k^{2}+28 k+23\right)\left(8 k^{3}+44 k^{2}+79 k+46\right)}
\end{aligned}
$$

Proof of Lemma 3.3. Based on the timeline in the Model ET under sequential setting, solving the first-order condition of the platform owner's profit from equation (3.12) with respect to $q_{p}^{\mathrm{ET}}$ yields $q_{p}^{\mathrm{ET}}=\frac{1-c_{p}-q_{r}^{\mathrm{ET}}-w_{p}^{\mathrm{ET}}}{2 k+2}$. After substituting $q_{p}^{\mathrm{ET}^{*}}\left(w_{p}^{\mathrm{ET}}, q_{r}^{\mathrm{ET}}\right)$ into equation $(3.11)$, the traditional retailer's profit is given: $\pi_{r}^{\mathrm{ET}}{ }^{*}=$ $\left(q_{r}^{\mathrm{ET}}\left(-2 c_{r}+c_{p}+2 k-q_{r}^{\mathrm{ET}}+w_{p}^{\mathrm{ET}}-2 w_{r}^{\mathrm{ET}}-2 c_{r} k-2 k q_{r}^{\mathrm{ET}}-2 k w_{r}^{\mathrm{ET}}-1\right)\right) / 2(k+1)$.

By applying the first-order condition to $\pi_{r}^{\mathrm{ET}}{ }^{*}$ with respect to $q_{r}^{\mathrm{ET}}$, we can obtain $q_{r}^{\mathrm{ET}}=$ $\left(c_{p}-2 c_{r}+2 k+w_{p}^{\mathrm{ET}}-2 w_{r}^{\mathrm{ET}}-2 c_{r} k-2 k w_{r}^{\mathrm{ET}}+1\right) /(4 k+2)$. Anticipating the traditional retailer's response $q_{r}^{\mathrm{ET} *}\left(w_{r}^{\mathrm{ET}}, w_{P}^{\mathrm{ET}}\right)$, the supplier chooses optimal wholesale price to maximise its profit. In other words,

$$
\max _{w_{r}^{\mathrm{ET}}, w_{P}^{\mathrm{ET}}} \pi_{s}^{\mathrm{ET}}=\frac{\left[\begin{array}{l}
c_{p}+2 w_{r}^{\mathrm{ET}}-4 k^{2} w_{r}^{\mathrm{ET}}-3 c_{p} w_{p}^{\mathrm{ET}}+2 c_{p} w_{r}^{\mathrm{ET}}+2 c_{r} w_{p}^{\mathrm{ET}}-4 c_{r} w_{r}^{\mathrm{ET}}+2 k w_{p}^{\mathrm{ET}} \\
+6 k w_{r}^{\mathrm{ET}}+4 w_{p}^{\mathrm{ET}} w_{r}^{\mathrm{ET}}-4 k w_{p}^{\mathrm{ET}}-8 k w_{r}^{\mathrm{ET}}+4 k^{2} w_{r}^{\mathrm{ET}}-3 w_{p}^{\mathrm{ET}^{2}}-4 w_{r}^{\mathrm{ET}} \\
-4 c_{r} k^{2} w_{r}^{\mathrm{ET}}-4 c_{p} k w_{p}^{\mathrm{ET}}+2 c_{p} k w_{r}^{\mathrm{ET}}+2 c_{r} k w_{p}^{\mathrm{ET}}-8 c_{r} k w_{r}^{\mathrm{ET}}+4 k w_{p}^{\mathrm{ET}} w_{r}^{\mathrm{ET}}
\end{array}\right]}{4\left(2 k^{2}+3 k+1\right)},
$$


and this expression yields $w_{r}^{\mathrm{ET}}{ }^{*}=\frac{1-c_{r}}{2}$ and $w_{P}^{\mathrm{ET}^{*}}=\frac{1-c_{p}}{2}$. Furthermore, substituting the optimal wholesale prices $w_{r}^{\mathrm{ET}^{*}}$ and $w_{P}^{\mathrm{ET}}$ into $q_{r}^{\mathrm{ET}}\left(w_{r}^{\mathrm{ET}}, w_{P}^{\mathrm{ET}}\right)$ and $q_{p}^{\mathrm{ET}}\left(w_{p}^{\mathrm{ET}}, q_{r}^{\mathrm{ET}}\right)$, we can derive the optimal quantities. That is,

$$
q_{r}^{\mathrm{ET}^{*}}=\frac{c_{p}-2 c_{r}+2 k-2 c_{r} k+1}{8 k+4} \quad \text { and } \quad q_{p}^{\mathrm{ET}^{*}}=\frac{2 c_{r}-3 c_{p}+2 k-4 c_{p} k+2 c_{r} k+1}{16 k^{2}+24 k+8} .
$$

Substituting the optima $w_{r}^{\mathrm{ET}}, w_{P}^{\mathrm{ET}}, q_{p}^{\mathrm{ET}^{*}}$ and $q_{r}^{\mathrm{ET}}{ }^{*}$ into equations (3.10), (3.11) and (3.12), we have the profits of all players as follow:

$$
\begin{aligned}
\pi_{s}^{\mathrm{ET}^{*}} & =\frac{\left[\begin{array}{l}
4 c_{p}^{2} k+3 c_{p}^{2}-4 c_{p} c_{r} k-4 c_{p} c_{r}-4 c_{p} k-2 c_{p}+4 c_{r}^{2} k^{2}+8 c_{r}^{2} k+4 c r^{2} \\
-8 c_{r} k^{2}-12 c_{r} k-4 c_{r}+4 k^{2}+8 k+3
\end{array}\right]}{16\left(2 k^{2}+3 k+1\right)}, \\
\pi_{p}^{\mathrm{ET}} & =\frac{\left(2 c_{r}-3 c_{p}+2 k-4 c_{p} k+2 c_{r} k+1\right)^{2}}{64(2 k+1)^{2}(k+1)} \text { and } \pi_{r}^{\mathrm{ET}}=\frac{\left(c_{p}-2 c_{r}+2 k-2 c_{r} k+1\right)^{2}}{32\left(2 k^{2}+3 k+1\right)} .
\end{aligned}
$$

Proof of Lemma 3.4. Based on the timeline in the Model EB under sequential setting, solving the first-order conditions of the supplier's profit from equation (3.15) with respect to $q_{s}^{\mathrm{EB}}$, and the platform owner's profit from equation (3.17) with respect to $q_{p}^{\mathrm{EB}}$, we have

$$
\begin{aligned}
q_{s}^{\mathrm{EB}} & =\frac{c_{p}-2 f^{\mathrm{EB}}+2 k-q_{r}^{\mathrm{EB}}+w_{p}^{\mathrm{EB}}-2 f^{\mathrm{EB}} k-2 k q_{r}^{\mathrm{EB}}+1}{2 k+3} \text { and } \\
q_{p}^{\mathrm{EB}} & =\frac{1-2 c_{p}+f^{\mathrm{EB}}-2 k-q_{r}^{\mathrm{EB}}-2 w_{p}^{\mathrm{EB}}+2 f^{\mathrm{EB}} k+2 k q_{r}^{\mathrm{EB}}}{2 k+3} .
\end{aligned}
$$

After substituting $q_{s}^{\mathrm{EB}}{ }^{*}\left(w_{P}^{\mathrm{EB}}, f^{\mathrm{EB}}, q_{r}^{\mathrm{EB}}\right)$ and $q_{p}^{\mathrm{EB}}{ }^{*}\left(w_{P}^{\mathrm{EB}}, f^{\mathrm{EB}}, q_{r}^{\mathrm{EB}}\right)$ into equation (3.17), the platform owner's profit is given:

$$
\pi_{p}^{\mathrm{EB}}=\frac{\left[\begin{array}{l}
c_{p}^{2} k+c_{p}^{2}+4 c_{p} f^{\mathrm{EB}} k+5 c_{p} f^{\mathrm{EB}}+6 c_{p} k q_{r}^{\mathrm{EB}}+4 c_{p} k w_{P}^{\mathrm{EB}}-6 c_{p} k+7 c_{p} q_{r}^{\mathrm{EB}}+5 c_{p} w_{P}^{\mathrm{EB}} \\
-7 c_{p}-4 f^{\mathrm{EB}^{2}} k^{2}-9 f^{\mathrm{EB}} k^{2}-5 f^{\mathrm{EB}^{2}}-8 f^{\mathrm{EB}} k^{2} q_{r}^{\mathrm{EB}}-4 f^{\mathrm{EB}} k^{2} w_{P}^{\mathrm{EB}}+8 f^{\mathrm{EB}} k^{2} \\
-14 f^{\mathrm{EB}} k q_{r}^{\mathrm{EB}}-6 f^{\mathrm{EB}} k w_{P}^{\mathrm{EB}}+14 f^{\mathrm{EB}} k-5 f^{\mathrm{EB}} q_{r}^{\mathrm{EB}}-f w_{P}^{\mathrm{EB}}+5 f^{\mathrm{EB}^{2}}-4 k^{2} q_{r}^{\mathrm{EB}} \\
-4 k^{2} q_{r}^{\mathrm{EB}} w_{P}^{\mathrm{EB}}+8 k^{2} q_{r}^{\mathrm{EB}}+4 k^{2} w_{P}^{\mathrm{EB}}-4 k^{2}-4 k q_{r}^{\mathrm{EB}}+4 w_{P}^{\mathrm{EB}}+1-2 k q_{r}^{\mathrm{EB}} w_{P}^{\mathrm{EB}} \\
+8 k q_{r}^{\mathrm{EB}}+3 k w_{P}^{\mathrm{EB}}+2 k w_{P}^{\mathrm{EB}}-4 k+q_{r}^{\mathrm{EB}}+4 q_{r}^{\mathrm{EB}} w_{P}^{\mathrm{EB}}-2 q_{r}^{\mathrm{EB}}-4 w_{P}^{\mathrm{EB}}
\end{array}\right]}{(2 k+3)^{2}},
$$

by applying the first-order condition to it with respect to $f^{\mathrm{EB}}$, we can obtain

$$
f^{\mathrm{EB}}{ }^{*}=\frac{-\left(5 q_{r}^{\mathrm{EB}}-14 k-5 c_{p}+w_{P}^{\mathrm{EB}}-4 c_{p} k+14 k q_{r}^{\mathrm{EB}}+6 k w_{P}^{\mathrm{EB}}+8 k^{2} q_{r}^{\mathrm{EB}}+4 k^{2} w_{P}^{\mathrm{EB}}-8 k^{2}-5\right)}{8 k^{2}+18 k+10} .
$$

Plugging $f^{\mathrm{EB}}{ }^{*}\left(w_{P}^{\mathrm{EB}}, q_{r}^{\mathrm{EB}}\right), q_{s}^{\mathrm{EB}}{ }^{*}\left(w_{P}^{\mathrm{EB}}, f^{\mathrm{EB}}, q_{r}^{\mathrm{EB}}\right)$ and $q_{p}^{\mathrm{EB}}{ }^{*}\left(w_{P}^{\mathrm{EB}}, f^{\mathrm{EB}}, q_{r}^{\mathrm{EB}}\right)$ into the traditional retailer's profit, the problem of the traditional retailer is given:

$$
\pi_{r}^{\mathrm{EB}}=\frac{\left[\begin{array}{l}
q_{r}\left(-10 c_{r}+5 c_{p}+14 k-5 q_{r}^{\mathrm{EB}}+3 w_{P}^{\mathrm{EB}}-10 w_{r}^{\mathrm{EB}}+4 c_{p} k-18 c_{r} k\right. \\
\left.-14 k q_{r}^{\mathrm{EB}}+2 k w_{P}^{\mathrm{EB}}-18 k w_{r}^{\mathrm{EB}}-8 c_{r} k^{2}-8 k^{2} q_{r}^{\mathrm{EB}}-8 k^{2} w_{r}^{\mathrm{EB}}+8 k^{2}+5\right)
\end{array}\right]}{8 k^{2}+18 k+10} .
$$


Solving the first-order condition of the traditional retailer' profit yields

$$
q_{r}^{\mathrm{EB}}{ }^{*}=\frac{5 c_{p}-10 c_{r}+14 k+3 w_{P}^{\mathrm{EB}}-10 w_{r}^{\mathrm{EB}}+4 c_{p} k-18 c_{r} k+2 k w_{P}^{\mathrm{EB}}-18 k w_{r}^{\mathrm{EB}}-8 c_{r} k^{2}-8 k^{2} w_{r}^{\mathrm{EB}}+8 k^{2}+5}{16 k^{2}+28 k+10} .
$$

Plugging $q_{r}^{\mathrm{EB}}{ }^{*}\left(w_{r}^{\mathrm{EB}}, w_{P}^{\mathrm{EB}}\right), f^{\mathrm{EB}}{ }^{*}\left(w_{P}^{\mathrm{EB}}, q_{r}^{\mathrm{EB}}\right), q_{s}^{\mathrm{EB}}{ }^{*}\left(w_{P}^{\mathrm{EB}}, f^{\mathrm{EB}}, q_{r}^{\mathrm{EB}}\right)$ and $q_{p}^{\mathrm{EB}}\left(w_{P}^{\mathrm{EB}}, f^{\mathrm{EB}}, q_{r}^{\mathrm{EB}}\right)$ into the supplier's profit, we have

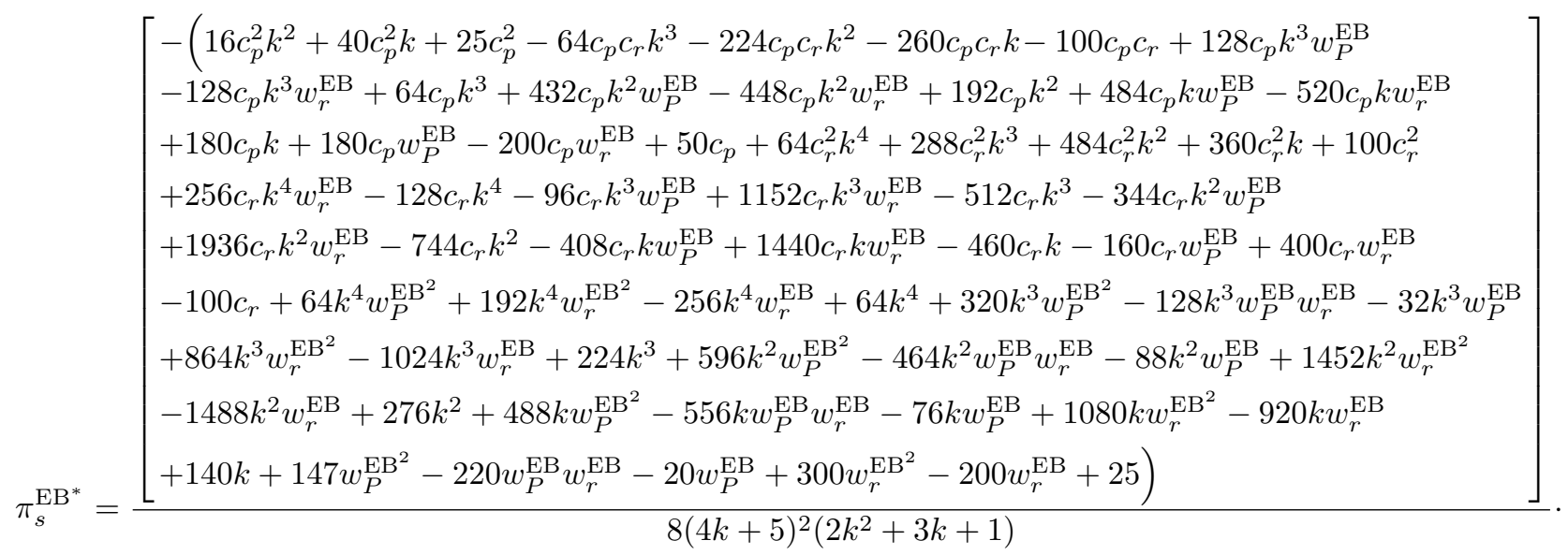

Because the objective function is jointly concave in $w_{r}^{\mathrm{EB}}$ and $w_{P}^{\mathrm{EB}}$, we can easily show that the optimal $w_{r}^{\mathrm{EB}}$ and $w_{P}^{\mathrm{EB}}$ are

$$
\begin{aligned}
w_{r}^{\mathrm{EB}} & =\frac{\left[\begin{array}{l}
9 c_{p}-98 c_{r}+358 k+56 c_{p} k-414 c_{r} k+80 c_{p} k^{2}+32 c_{p} k^{3}-568 c_{r} k^{2} \\
-320 c_{r} k^{3}-64 c_{r} k^{4}+488 k^{2}+288 k^{3}+64 k^{4}+89
\end{array}\right]}{4\left(32 k^{4}+160 k^{3}+287 k^{2}+214 k+53\right)} \text { and } \\
w_{p}^{\mathrm{EB}} & =\frac{\left[\begin{array}{l}
10 c_{r}-55 c_{p}+161 k-189 c_{p} k+28 c_{r} k-196 c_{p} k^{2} \\
-64 c_{p} k^{3}+26 c_{r} k^{2}+8 c_{r} k^{3}+170 k^{2}+56 k^{3}+45
\end{array}\right]}{2\left(32 k^{4}+160 k^{3}+287 k^{2}+214 k+53\right)},
\end{aligned}
$$

respectively. Furthermore, substituting the optimal wholesale prices $w_{r}^{\mathrm{EB}}$ and $w_{p}^{\mathrm{EB}}{ }^{*}$ into $q_{r}^{\mathrm{EB}}{ }^{*}\left(w_{r}^{\mathrm{EB}}, w_{P}^{\mathrm{EB}}\right)$, $f^{\mathrm{EB}}\left(w_{P}^{\mathrm{EB}}, q_{r}^{\mathrm{EB}}\right), q_{s}^{\mathrm{EB}}\left(w_{P}^{\mathrm{EB}}, f^{\mathrm{EB}}, q_{r}^{\mathrm{EB}}\right)$ and $q_{p}^{\mathrm{EB}}\left(w_{P}^{\mathrm{EB}}, f^{\mathrm{EB}}, q_{r}^{\mathrm{EB}}\right)$, we can derive the optimal quantities and commission fee. That is,

$$
\begin{aligned}
q_{r}^{\mathrm{EB}} & =\frac{\left[\begin{array}{l}
32 c_{p}-54 c_{r}+99 k+63 c_{p} k-162 c_{r} k+40 c_{p} k^{2}+8 c_{p} k^{3} \\
-180 c_{r} k^{2}-88 c_{r} k^{3}-16 c_{r} k^{4}+140 k^{2}+80 k^{3}+16 k^{4}+22
\end{array}\right]}{2\left(32 k^{4}+287 k^{2}+160 k^{3}+53+214 k\right)}, \\
f^{\mathrm{EB}^{*}} & =\frac{\left[\begin{array}{l}
85 c_{p}+52 c_{r}+418 k+396 c_{p} k+254 c_{r} k+642 c_{p} k^{2}+444 c_{p} k^{3}+112 c_{p} k^{4} \\
+470 c_{r} k^{2}+420 c_{r} k^{3}+184 c_{r} k^{4}+32 c_{r} k^{5}+892 k^{2}+924 k^{3}+472 k^{4}+96 k^{5}+75
\end{array}\right]}{4\left(32 k^{5}+192 k^{4}+447 k^{3}+501 k^{2}+267 k+53\right)}, \\
q_{s}^{\mathrm{EB}^{*}} & =\frac{(k+1)\left(2 c_{r}-11 c_{p}+25 k-29 c_{p} k+4 c_{r} k-16 c_{p} k^{2}+2 c_{r} k^{2}+14 k^{2}+9\right)}{32 k^{4}+160 k^{3}+287 k^{2}+214 k+53} \text { and }
\end{aligned}
$$




$$
q_{P}^{\mathrm{EB}^{*}}=\frac{\left[\begin{array}{l}
40 c_{r}-61 c_{p}+64 k-178 c_{p} k+114 c_{r} k-168 c_{p} k^{2}-52 c_{p} k^{3} \\
+118 c_{r} k^{2}+52 c_{r} k^{3}+8 c_{r} k^{4}+50 k^{2}-8 k^{4}+21
\end{array}\right]}{4\left(32 k^{5}+192 k^{4}+447 k^{3}+501 k^{2}+267 k+53\right)} .
$$

Substituting the optimal $w_{r}^{\mathrm{EB}}, w_{p}^{\mathrm{EB}}, q_{r}^{\mathrm{EB}^{*}}, f^{\mathrm{EB}^{*}}, q_{s}^{\mathrm{EB}}{ }^{*}$ and $q_{P}^{\mathrm{EB}}{ }^{*}$ into Equation (3.15), (3.16) and (3.17), we have the profits of all players as follow:

$$
\begin{aligned}
& \pi_{s}^{\mathrm{EB}^{*}}=\frac{\left[\begin{array}{l}
72 c_{p}^{2} k^{3}+236 c_{p}^{2} k^{2}+252 c_{p}^{2} k+87 c_{p}^{2}-32 c_{p} c_{r} k^{4}-192 c_{p} c_{r} k^{3}-412 c_{p} c_{r} k^{2}-380 c_{p} c_{r} k-128 c_{p} c_{r} \\
+32 c_{p} k^{4}+48 c_{p} k^{3}-60 c_{p} k^{2}-124 c_{p} k-46 c_{p}+32 c_{r}^{2} k^{5}+208 c_{r}^{2} k^{4}+536 c_{r}^{2} k^{3}+684 c_{r}^{2} k^{2}+432 c_{r}^{2} k \\
+108 c_{r}^{2}-64 c_{r} k^{5}-384 c_{r} k^{4}-880 c_{r} k^{3}-956 c_{r} k^{2}-484 c_{r} k-88 c_{r}+32 k^{5}+176 k^{4}+416 k^{3} \\
+508 k^{2}+304 k+67
\end{array}\right]}{8(k+1)\left(32 k^{4}+287 k^{2}+160 k^{3}+53+214 k\right)}, \\
& \pi_{P}^{\mathrm{EB}^{*}}=\frac{\left[\begin{array}{l}
(2 k+3)\left(2048 c_{p}^{2} k^{7}+15872 c_{p}^{2} k^{6}+51872 c_{p}^{2} k^{5}+92712 c_{p}^{2} k^{4}+98000 c_{p}^{2} k^{3}+61388 c_{p}^{2} k^{2}+21154 c_{p}^{2} k\right. \\
+3103 c_{p}^{2}-1024 c_{p} c_{r} k^{7}-8320 c_{p} c_{r} k^{6}-28800 c_{p} c_{r} k^{5}-55160 c_{p} c_{r} k^{4}-63256 c_{p} c_{r} k^{3}-43508 c_{p} c_{r} k^{2} \\
-16636 c_{p} c_{r} k-2728 c_{p} c_{r}-3072 c_{p} k^{7}-23424 c_{p} k^{6}-74944 c_{p} k^{5}-130264 c_{p} k^{4}-132744 c_{p} k^{3} \\
-79268 c_{p} k^{2}-25672 c_{p} k-3478 c_{p}+128 c_{r}^{2} k^{7}+1120 c_{r}^{2} k^{6}+4216 c_{r}^{2} k^{5}+8860 c_{r}^{2} k^{4}+11232 c_{r}^{2} k^{3} \\
+8588 c_{r}^{2} k^{2}+3664 c_{r}^{2} k+672 c_{r}^{2}+768 c_{r} k^{7}+6080 c_{r} k^{6}+20368 c_{r} k^{5}+37440 c_{r} k^{4}+40792 c_{r} k^{3} \\
+26332 c_{r} k^{2}+9308 c_{r} k+1384 c_{r}+1152 k^{7}+8672 k^{6}+27288 k^{5}+46412 k^{4}+45976 k^{3}+26468 k^{2} \\
+8182 k+1047)
\end{array}\right]}{16(k+1)\left(32 k^{4}+160 k^{3}+287 k^{2}+214 k+53\right)^{2}}
\end{aligned}
$$

and

$$
\pi_{r}^{\mathrm{EB}^{*}}=\frac{\left[\begin{array}{l}
(2 k+1)\left(32 c_{p}-54 c_{r}+99 k+63 c_{p} k-162 c_{r} k+40 c_{p} k^{2}+8 c_{p} k^{3}\right. \\
\left.-180 c_{r} k^{2}-88 c_{r} k^{3}-16 c_{r} k^{4}+140 k^{2}+80 k^{3}+16 k^{4}+22\right)^{2}
\end{array}\right]}{4\left(64 k^{4}+320 k^{3}+574 k^{2}+428 k+106\right)\left(32 k^{5}+192 k^{4}+447 k^{3}+501 k^{2}+267 k+53\right)} .
$$

All parameters and variables in this paper must satisfy non-negativity constraints, that is, under sequential setting, we only consider $\frac{2 k^{2} c_{r}-2 k^{2}+8 k c_{r}-7 k+8 c_{r}-5}{k+3}<c_{p}<\frac{2 k c_{r}+6 k+4 c_{r}+9}{8 k+13}$ in the scenarios of Model EM (referred to as $\frac{c_{p}}{p}=\frac{2 k^{2} c_{r}-2 k^{2}+8 k c_{r}-7 k+8 c_{r}-5}{k+3}, \overline{c_{p}}=\frac{2 k c_{r}+6 k+4 c_{r}+9}{8 k+13}$ ), and $\left[\begin{array}{l}16 c_{r} k^{4}+88 c_{r} k^{3}-16 k^{4}+180 c_{r} k^{2}-80 k^{3} \\ +162 c_{r}-140 k^{2}+54 c_{r}-99 k-22\end{array}\right]\left[\begin{array}{l}8 c_{r} k^{4}+52 c_{r} k^{3}-8 k^{4}+118 c_{r} k^{2}+114 c_{r} k \\ +50 k^{2}+40 c_{r}+64 k+21\end{array}\right]$

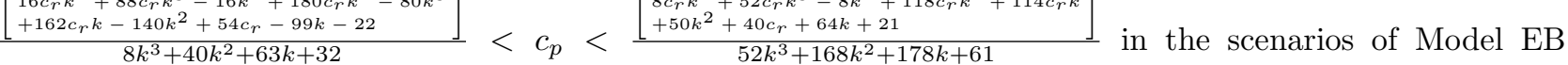
(referred to as $c_{p}=\frac{\left[\begin{array}{l}16 c_{r} k^{4}+88 c_{r} k^{3}-16 k^{4}+180 c_{r} k^{2}-80 k^{3} \\ +162 c_{r} k-140 k^{2}+54 c_{r}-99 k-22\end{array}\right]}{8 k^{3}+40 k^{2}+63 k+32}, \overline{\overline{c_{p}}}=\frac{\left[\begin{array}{l}8 c_{r} k^{4}+52 c_{r} k^{3}-8 k^{4}+118 c_{r} k^{2}+114 c_{r} k \\ +50 k^{2}+40 c_{r}+64 k+21\end{array}\right]}{52 k^{3}+168 k^{2}+178 k+61}$ ). Under these constraints, we compare the outcomes among Model N, Model EM, Model ET and Model EB and draw the Propositions in the sequential setting.

Proof of Proposition 4.1. Based on the outcomes of Lemmas 3.1 and 3.2, we compare the supplier's profits between Model EM and Model N:

$$
\pi_{s}^{\mathrm{EM}^{*}}-\pi_{s}^{\mathrm{N}^{*}}=\frac{\left[\begin{array}{l}
18 c_{p}^{2} k+32 c_{p}^{2}-8 c_{p} c_{r} k^{2}-40 c_{p} c_{r} k-48 c_{p} c_{r}+8 c_{p} k^{2}+4 c_{p} k \\
-16 c_{p}+4 c_{r}^{2} k^{2}+17 c_{r}^{2} k+18 c_{r}^{2}+6 c_{r} k+12 c_{r}-4 k^{2}-5 k+2
\end{array}\right]}{8\left(8 k^{3}+44 k^{2}+79 k+46\right)} .
$$


$\pi_{s}^{\mathrm{EM}^{*}}-\pi_{s}^{\mathrm{N}^{*}}$ is a quadratic function of $c_{p}$, and the coefficient $\frac{16+9 k}{4(2+k)\left(8 k^{2}+28 k+23\right)}$ is positive, which shows that the equation of $\pi_{s}^{\mathrm{EM}^{*}}-\pi_{s}^{\mathrm{N}^{*}}$ is a convex function of $c_{p}$. Further, solving $\pi_{s}^{\mathrm{EM}^{*}}-\pi_{s}^{\mathrm{N}^{*}}=0$, we obtain that there exists two roots (with respect to $c_{p}$ ):

$$
c_{p 1}=\frac{24 c_{r}-2 k-\left(2 k(k+2)\left(8 k^{2}+28 k+23\right)\right)^{\frac{1}{2}}+20 c_{r} k+4 c_{r} k^{2}-4 k^{2}+c_{r}\left(2 k(k+2)\left(8 k^{2}+28 k+23\right)\right)^{\frac{1}{2}}+8}{2(18 k+32)}
$$

and

$$
c_{p 2}=\frac{24 c_{r}-2 k+\left(2 k(k+2)\left(8 k^{2}+28 k+23\right)\right)^{\frac{1}{2}}+20 c_{r} k+4 c_{r} k^{2}-4 k^{2}-c_{r}\left(2 k(k+2)\left(8 k^{2}+28 k+23\right)\right)^{\frac{1}{2}}+8}{2(9 k+16)} .
$$

This, together with the fact that $\underline{c_{p}}<c_{p 1}<c_{p 2}<\overline{c_{p}}$, suggests that $\pi_{s}^{\mathrm{EM}^{*}}>\pi_{s}^{\mathrm{N}^{*}}$ when $c_{p}<c_{p 1}$ or $c_{p}>c_{p 2}$. Otherwise, $\pi_{s}^{\mathrm{EM}^{*}}<\pi_{s}^{\mathrm{N}^{*}}$.

Then, comparing the traditional retailer's profits between Model EM and Model N, we can obtain:

$$
\pi_{r}^{\mathrm{EM}^{*}}-\pi_{r}^{\mathrm{N}^{*}}=\frac{\left[\begin{array}{l}
16 c_{p}^{2} k^{3}+120 c_{p}^{2} k^{2}+288 c_{p}^{2} k+216 c_{p}^{2}-64 c_{p} c_{r} k^{4}-544 c_{p} c_{r} k^{3}-1696 c_{p} c_{r} k^{2} \\
-2304 c_{p} c_{r} k-1152 c_{p} c_{r}+64 c_{p} k^{4}+512 c_{p} k^{3}+1456 c_{p} k^{2}+1728 c_{p} k+720 c_{p} \\
+32 c_{r}^{2} k^{4}+256 c_{r}^{2} k^{3}+760 c_{r}^{2} k^{2}+991 c_{r}^{2} k+478 c_{r}^{2}+32 c_{r} k^{3}+176 c_{r} k^{2}+322 c_{r} k \\
+196 c_{r}-32 k^{4}-272 k^{3}-816 k^{2}-1025 k-458
\end{array}\right]}{16(k+2)\left(8 k^{2}+28 k+23\right)^{2}} .
$$

$\pi_{r}^{\mathrm{EM}^{*}}-\pi_{r}^{\mathrm{N}^{*}}$ is also a quadratic function of $c_{p}$, and the coefficient $\frac{(2 k+3)(k+3)^{2}}{\left(16 k^{2}+56 k+46\right)\left(8 k^{3}+44 k^{2}+79 k+46\right)}$ is positive, which shows that the equation of $\pi_{r}^{\mathrm{EM}^{*}}-\pi_{r}^{\mathrm{N}^{*}}$ is a convex function of $c_{p}$. There are two roots (with respect to $c_{p}$ ) for the equation $\pi_{r}^{\mathrm{EM}^{*}}-\pi_{r}^{\mathrm{N}^{*}}=0$ :

$$
c_{p 3}=\frac{-\left(7 k-8 c_{r}-8 c_{r} k-2 c_{r} k^{2}+2 k^{2}+\left(2^{\frac{1}{2}}\left(c_{r}-1\right)(k+2)^{\frac{1}{2}}\left(8 k^{2}+28 k+23\right)\right) /\left(4(2 k+3)^{\frac{1}{2}}\right)+5\right)}{k+3}
$$

and

$$
c_{p 3}^{*}=\frac{8 c_{r}-7 k+8 c_{r} k+2 c_{r} k^{2}-2 k^{2}+\left(2^{\frac{1}{2}}\left(c_{r}-1\right)(k+2)^{\frac{1}{2}}\left(8 k^{2}+28 k+23\right)\right) /\left(4(2 k+3)^{\frac{1}{2}}\right)-5}{k+3} .
$$

After calculation, we can find $c_{p 3}^{*}<c_{p}<c_{p 3}<\overline{c_{p}}$. Thus, when $c_{p}>c_{p 3}$, the supplier encroachment always increases the traditional retailer's profit, i.e., $\pi_{r}^{\mathrm{EM}^{*}}>\pi_{r}^{\mathrm{N}^{*}}$; Otherwise $\pi_{r}^{\mathrm{EM}^{*}}<\pi_{r}^{\mathrm{N}^{*}}$. Moreover, since $c_{p 2}<c_{p 3}$, we can easily show that $\pi_{s}^{\mathrm{EM}^{*}}>\pi_{s}^{\mathrm{N}^{*}}$ and $\pi_{r}^{\mathrm{EM}^{*}}>\pi_{r}^{\mathrm{N}^{*}}$ when $c_{p}>c_{p 3} ; \pi_{s}^{\mathrm{EM}^{*}}>\pi_{s}^{\mathrm{N}^{*}}$ and $\pi_{r}^{\mathrm{EM}^{*}}<\pi_{r}^{\mathrm{N}^{*}}$ when $c_{p}<c_{p 1}$ or $c_{p 2}<c_{p}<c_{p 3} ; \pi_{s}^{\mathrm{EM}^{*}}<\pi_{s}^{\mathrm{N}^{*}}$ and $\pi_{r}^{\mathrm{EM}^{*}}<\pi_{r}^{\mathrm{N}^{*}}$ when $c_{p 1}<c_{p}<c_{p 2}$.

Proof of Proposition 4.2. Based on the outcomes of Lemmas 3.1 and 3.4, we compare the supplier's profits between Model EB and Model N:

$$
\pi_{s}^{\mathrm{EB}^{*}}-\pi_{s}^{\mathrm{N}^{*}}=\frac{\left[\begin{array}{l}
\left(72 k^{3}+236 k^{2}+252 k+87\right) c_{p}^{2}-\left(128 c_{r}+124 k+380 c_{r} k+412 c_{r} k^{2}+192 c_{r} k^{3}\right. \\
\left.+32 c_{r} k^{4}+60 k^{2}-48 k^{3}-32 k^{4}+46\right) c_{p}+16 c_{r}^{2} k^{4}+89 c_{r}^{2} k^{3}+183 c_{r}^{2} k^{2}+165 c_{r}^{2} k \\
+55 c_{r}^{2}+14 c_{r} k^{3}+46 c_{r} k^{2}+50 c_{r} k+18 c_{r}-16 k^{4}-31 k^{3}+7 k^{2}+37 k+14
\end{array}\right]}{8\left(32 k^{5}+192 k^{4}+447 k^{3}+501 k^{2}+267 k+53\right)} .
$$


We find that $\pi_{s}^{\mathrm{EB}^{*}}-\pi_{s}^{\mathrm{N}^{*}}$ is a quadratic function of $c_{p}$, that is $\pi_{s}^{\mathrm{EB}^{*}}-\pi_{s}^{\mathrm{N}^{*}}=A c_{p}^{2}+\mathrm{B} c_{p}+$ $C$, where $A=\frac{72 k^{3}+236 k^{2}+252 k+87}{8\left(32 k^{5}+192 k^{4}+447 k^{3}+501 k^{2}+267 k+53\right)}, B=\frac{\left[\begin{array}{l}-128 c_{r}-124 k-380 c_{r} k-412 c_{r} k^{2}-192 c_{r} k^{3} \\ -32 k^{4}-60 k^{2}+48 k^{3}+32 k^{4}-46\end{array}\right]}{8\left(32 k^{5}+192 k^{4}+447 k^{3}+501 k^{2}+267 k+53\right)}$ and $C=$ $\left[16 c_{r}^{2} k^{4}+89 c_{r}^{2} k^{3}+183 c_{r}^{2} k^{2}+165 c_{r}^{2} k+55 c_{r}^{2}+14 c_{r} k^{3}\right]$

$\frac{\left[46 c_{r} k^{2}+50 c_{r} k+18 c_{r}-16 k^{4}-3 c^{3}+7 k^{2}+37 k+14\right.}{8\left(32 k^{5}+192 k^{4}+447 k^{3}+501 k^{2}+267 k+53\right)}$. We obtain that the coefficient $A$ is positive. That means the equa$8\left(32 k^{5}+192 k^{4}+447 k^{3}+501 k^{2}+267 k+53\right)$
tion of $\pi_{s}^{\mathrm{EB}^{*}}-\pi_{s}^{\mathrm{N}^{*}}$ is a convex function of $c_{p}$. Further, after calculation, we find that $B^{2}-4 A C<0$ for any $k$
and $c_{r}$, that means there is no root (with respect to $c_{p}$ ) for the equation $\pi_{s}^{\mathrm{EB}^{*}}-\pi_{s}^{\mathrm{N}^{*}}=0$. Thus, $\pi_{s}^{\mathrm{EB}^{*}}-\pi_{s}^{\mathrm{N}^{*}}>0$ always holds for any $c_{p}$.

Similarly, we compare the traditional retailer's profits between Model EB and Model N:

$$
\pi_{r}^{\mathrm{EB}}-\pi_{r}^{\mathrm{N}^{*}}=\frac{\left[\begin{array}{l}
(2 k+1)\left(32 c_{p}-54 c_{r}+99 k+63 c_{p} k-162 c_{r} k+40 c_{p} k^{2}+8 c_{p} k^{3}\right. \\
\left.-180 c_{r} k^{2}-88 c_{r} k^{3}-16 c_{r} k^{4}+140 k^{2}+80 k^{3}+16 k^{4}+22\right)^{2}
\end{array}\right]}{8\left(32 k^{4}+160 k^{3}+287 k^{2}+214 k+53\right)\left(32 k^{5}+192 k^{4}+447 k^{3}+501 k^{2}+267 k+53\right)}-\frac{\left(c_{r}-1\right)^{2}}{16} .
$$

$\pi_{r}^{\mathrm{EB}^{*}}-\pi_{r}^{\mathrm{N}^{*}}$ is a quadratic function of $c_{p}$, and the coefficient $\frac{(2 k+1)\left(8 k^{3}+40 k^{2}+63 k+32\right)^{2}}{8(k+1)\left(32 k^{4}+287 k^{2}+160 k^{3}+53+214 k\right)^{2}}$ is positive. Then, there are two roots (with respect to $c_{p}$ ) for the equation $\pi_{r}^{\mathrm{EB}}-\pi_{r}^{\mathrm{N}^{*}}=0$ :

$$
c_{p}^{*}=\frac{\left[\begin{array}{l}
54 c_{r}-99 k+162 c_{r} k+180 c_{r} k^{2}+88 c_{r} k^{3}+16 c_{r} k^{4}-140 k^{2}-80 k^{3}-16 k^{4} \\
+\left(2^{\frac{1}{2}}\left(c_{r}-1\right)(k+1)^{\frac{1}{2}}\left(32 k^{4}+160 k^{3}+287 k^{2}+214 k+53\right)\right) /\left(2(2 k+1)^{\frac{1}{2}}\right)-22
\end{array}\right]}{32+63 k+40 k^{2}+8 k^{3}}
$$

and

$$
c_{p}^{* *}=\frac{\left[\begin{array}{l}
-\left(99 k-54 c_{r}-162 c_{r} k-180 c_{r} k^{2}-88 c_{r} k^{3}-16 c_{r} k^{4}+140 k^{2}+80 k^{3}+16 k^{4}\right. \\
\left.+\left(2^{\frac{1}{2}}\left(c_{r}-1\right)(k+1)^{\frac{1}{2}}\left(32 k^{4}+160 k^{3}+287 k^{2}+214 k+53\right)\right) /\left(2(2 k+1)^{\frac{1}{2}}\right)+22\right)
\end{array}\right]}{32+63 k+40 k^{2}+8 k^{3}} .
$$

This, together with the fact that $c_{p}^{* *}<\underline{\underline{c_{p}}}<\overline{\overline{c_{p}}}<c_{p}^{*}$, suggests that, for any $c_{p}, \pi_{r}^{\mathrm{EB}}-\pi_{r}^{\mathrm{N}^{*}}<0$ always holds.

Proof of Proposition 4.3. Based on the outcomes of Lemmas 3.3 and 3.4, we compare the supplier's profits between Model EB and Model ET:

$$
\pi_{s}^{\mathrm{EB}^{*}}-\pi_{s}^{\mathrm{ET}^{*}}=\frac{\left[\begin{array}{l}
-128 c_{p}^{2} k^{4}-320 c_{p}^{2} k^{3}-220 c_{p}^{2} k^{2}-17 c_{p}^{2} k-15 c_{p}^{2}-64 c_{p} c_{r} k^{3}-180 c_{p} c_{r} k^{2}-160 c_{p} c_{r} k \\
-44 c_{p} c_{r}+256 c_{p} k^{4}+704 c_{p} k^{3}+620 c_{p} k^{2}+194 c_{p} k+14 c_{p}+4 c_{r}^{2} k^{3}+12 c_{r}^{2} k^{2}+12 c_{r}^{2} k \\
+4 c r^{2}+56 c_{r} k^{3}+156 c_{r} k^{2}+136 c_{r} k+36 c_{r}-128 k^{4}-380 k^{3}-388 k^{2}-165 k-25
\end{array}\right]}{16\left(64 k^{5}+352 k^{4}+734 k^{3}+715 k^{2}+320 k+53\right)} .
$$

$\pi_{s}^{\mathrm{EB}^{*}}-\pi_{s}^{\mathrm{ET}^{*}}$ is a quadratic function of $c_{p}$, and the coefficient $\frac{-\left(256 k^{4}+640 k^{3}+440 k^{2}+34 k+30\right)}{1024 k^{5}+5632 k^{4}+11744 k^{3}+11440 k^{2}+5120 k+848}$ is negative, which shows that the equation of $\pi_{s}^{\mathrm{EB}^{*}}-\pi_{s}^{\mathrm{ET}}$ is a concave function of $c_{p}$. Further, solving $\pi_{s}^{\mathrm{EB}}{ }^{*}-\pi_{s}^{\mathrm{ET}}=$ 0 , we obtain that there exist two roots (with respect to $c_{p}$ ): 
$c_{p}^{* *}=-\frac{\left[\begin{array}{l}97 k-22 c_{r}-80 c_{r} k-90 c_{r} k^{2}-32 c_{r} k^{3}+310 k^{2}+352 k^{3}+128 k^{4}+2 \sqrt{2}\left(64 k^{5}+352 k^{4}+734 k^{3}\right. \\ \left.+715 k^{2}+320 k+53\right)^{\frac{1}{2}}-2 \sqrt{2} c_{r}\left(64 k^{5}+352 k^{4}+734 k^{3}+715 k^{2}+320 k+53\right)^{\frac{1}{2}}+2 \sqrt{2} k\left(64 k^{5}\right. \\ \left.+352 k^{4}+734 k^{3}+715 k^{2}+320 k+53\right)^{\frac{1}{2}}-2 \sqrt{2} c_{r} k\left(64 k^{5}+352 k^{4}+734 k^{3}+715 k^{2}+320 k+53\right)^{\frac{1}{2}} \\ +7\end{array}\right]}{128 k^{4}+320 k^{3}+220 k^{2}+17 k+1}$

and

$c_{p}^{*}=-\frac{\left[\begin{array}{l}97 k-22 c_{r}-80 c_{r} k-90 c_{r} k^{2}-32 c_{r} k^{3}+310 k^{2}+352 k^{3}+128 k^{4}-2 \sqrt{2}\left(64 k^{5}+352 k^{4}+734 k^{3}\right. \\ \left.+715 k^{2}+320 k+53\right)^{\frac{1}{2}}+2 \sqrt{2} c_{r}\left(64 k^{5}+352 k^{4}+734 k^{3}+715 k^{2}+320 k+53\right)^{\frac{1}{2}}-2 \sqrt{2} k\left(64 k^{5}\right. \\ \left.+352 k^{4}+734 k^{3}+715 k^{2}+320 k+53\right)^{\frac{1}{2}}+2 \sqrt{2} c_{r} k\left(64 k^{5}+352 k^{4}+734 k^{3}+715 k^{2}+320 k+53\right)^{\frac{1}{2}} \\ +7\end{array}\right]}{128 k^{4}+320 k^{3}+220 k^{2}+17 k+1}$.

This, together with the fact that $c_{p}^{*}<\underline{\underline{c_{p}}}<\overline{\overline{c_{p}}}<c_{p}^{* *}$, shows that, for any $c_{p}, \pi_{s}^{\mathrm{EB}^{*}}-\pi_{s}^{\mathrm{ET}}{ }^{*}>0$ always holds.

Next, we compare the traditional retailer's profits between Model EB and Model ET:

$$
\begin{aligned}
& \pi_{r}^{\mathrm{EB}^{*}}-\pi_{r}^{\mathrm{ET}^{*}}= \\
& {\left[\begin{array}{l}
1024 c_{p}^{2} k^{6}+7232 c_{p}^{2} k^{5}+20352 c_{p}^{2} k^{4}+29135 c_{p}^{2} k^{3}+22253 c_{p}^{2} k^{2}+8541 c_{p}^{2} * k+1287 c_{p}^{2}-2048 c_{p} c_{r} k^{7} \\
-16128 c_{p} c_{r} k^{6}-53248 c_{p} c_{r} k^{5}-95228 c_{p} c_{r} k^{4}-99248 c_{p} c_{r} k^{3}-60024 c_{p} c_{r} k^{2}-19424 c_{p} c_{r} k-2588 c_{p} c_{r} \\
+2048 c_{p} k^{7}+14080 c_{p} k^{6}+38784 c_{p} k^{5}+54524 c_{p} k^{4}+40978 c_{p} k^{3}+15518 c_{p} k^{2}+2342 c_{p} k+14 c_{p} \\
+256 c_{r}^{2} k^{7}+2048 c_{r}^{2} k^{6}+6908 c_{r}^{2} k^{5}+12716 c_{r}^{2} k^{4}+13768 c_{r}^{2} k^{3}+8744 c_{r}^{2} k^{2}+3004 c_{r}^{2} k+428 c_{r}^{2}+1536 c_{r} k^{7} \\
+12032 c_{r} k^{6}+39432 c_{r} k^{5}+69796 c_{r} k^{4}+71712 c_{r} k^{3}+42536 c_{r} k^{2}+13416 c_{r} k+1732 c_{r}-1792 k^{7} \\
-13056 k^{6}-39108 k^{5}-62160 k^{4}-56345 k^{3}-29027 k^{2}-7879 k-873
\end{array}\right] .} \\
& \frac{32(2 k+1)\left(32 k^{4}+160 k^{3}+287 k^{2}+214 k+53\right)^{2}}{}
\end{aligned}
$$

$\pi_{r}^{\mathrm{EB}^{*}}-\pi_{r}^{\mathrm{ET}} \mathrm{T}^{*}$ is a quadratic function of $c_{p}$, and the coefficient $\frac{1024 k^{6}+7232 k^{5}+20352 k^{4}+29135 k^{3}+22253 k^{2}+8541 k+1287}{16(2 k+1)\left(32 k^{4}+160 k^{3}+287 k^{2}+214 *+53\right)^{2}}$ is positive, which shows that the equation of $\pi_{r}^{\mathrm{EB}}{ }^{*}-\pi_{r}^{\mathrm{ET}}$ is a convex function of $c_{p}$. Further, solving $\pi_{r}^{\mathrm{EB}}{ }^{*}$ $\pi_{r}^{\mathrm{ET}}=0$, we obtain that there exist two roots (with respect to $c_{p}$ ):

$$
c_{p}^{* *}=\frac{2 c_{r}+25 k+4 c_{r} k+2 c_{r} k^{2}+14 k^{2}+9}{16 k^{2}+29 k+11}
$$

and

$$
c_{p}^{*}=\frac{\left[\begin{array}{l}
214 c_{r}-606 k+1074 c_{r} k+2010 c_{r} k^{2}+1790 c_{r} k^{3}+768 c_{r} k^{4} \\
+128 c_{r} k^{5}-1391 k^{2}-1454 k^{3}-704 k^{4}-128 k^{5}-97
\end{array}\right]}{64 k^{4}+336 k^{3}+619 k^{2}+468 k+117} .
$$

This, together with the fact that $c_{p}^{*}<\underline{\underline{c_{p}}}<\overline{\overline{c_{p}}}<c_{p}^{* *}$, shows that, for any $c_{p}, \pi_{r}^{\mathrm{EB}^{*}}-\pi_{r}^{\mathrm{ET}}{ }^{*}<0$ always holds. 
Additionally, we compare the platform owner's profits between Model EB and Model ET: $\pi_{P}^{\mathrm{EB}^{*}}-\pi_{P}^{\mathrm{ET}^{*}}=$

$\left[\begin{array}{l}49152 c_{p}^{2} k^{9}+434176 c_{p}^{2} k^{8}+1651712 c_{p}^{2} k^{7}+3542272 c_{p}^{2} k^{6}+4709552 c_{p}^{2} k^{5}+4020856 c_{p}^{2} k^{4}+2204895 c_{p}^{2} k^{3} \\ +750109 c_{p}^{2} k^{2}+144089 c_{p}^{2} k+11955 c_{p}^{2}-16384 c_{p} c_{r} k^{9}-139264 c_{p} c_{r} k^{8}-502784 c_{p} c_{r} k^{7}-1001984 c_{p} c_{r} k^{6} \\ -1197168 c_{p} c_{r} k^{5}-868212 c_{p} c_{r} k^{4}-364368 c_{p} c_{r} k^{3}-74728 c_{p} c_{r} k^{2}-2512 c_{p} c_{r} k+972 c_{p} c_{r}-81920 c_{p} k^{9} \\ -729088 c_{p} k^{8}-2800640 c_{p} k^{7}-6082560 c_{p} k^{6}-8221936 c_{p} k^{5}-7173500 c_{p} k^{4}-4045422 c_{p} k^{3} \\ -1425490 c_{p} k^{2}-285666 c_{p} k-24882 c_{p}-3072 c_{r}^{2} k^{8}-27136 c_{r}^{2} k^{7}-102656 c_{r}^{2} k^{6}-216708 c_{r}^{2} k^{5} \\ -278420 c_{r}^{2} k^{4}-222184 c_{r}^{2} k^{3}-107144 c_{r}^{2} k^{2}-28436 c_{r}^{2} k-3172 c_{r}^{2}+16384 c_{r} k^{9}+145408 c_{r} k^{8} \\ +557056 c_{r} k^{7}+1207296 c_{r} k^{6}+1630584 c_{r} k^{5}+1425052 c_{r} k^{4}+808736 c_{r} k^{3}+289016 c_{r} k^{2}+59384 c_{r} k \\ +5372 c_{r}+32768 k^{9}+291840 k^{8}+1121792 k^{7}+2437632 k^{6}+3295676 k^{5}+2874224 k^{4}+1618343 k^{3} \\ +568237 k^{2}+113141 k+9755\end{array}\right]$. $64\left(64 k^{5}+352 k^{4}+734 k^{3}+715 k^{2}+320 k+53\right)^{2}$

$\pi_{p}^{\mathrm{EB}^{*}}-\pi_{p}^{\mathrm{ET}^{*}}$ is a quadratic function of $c_{p}$, and the coefficient $\frac{\left[\begin{array}{c}49152 k^{9}+434176 k^{8}+1651712 k^{7}+3542272 k^{6}+4709552 k^{5} \\ +4020856 k^{4}+2204895 k^{3}+750109 k^{2}+144089 k+11955\end{array}\right]}{32\left(64 k^{5}+352 k^{4}+734 k^{3}+715 k^{2}+320 k+53\right)^{2}}$ is positive. That means the equation of $\pi_{p}^{\mathrm{EB}}-\pi_{p}^{\mathrm{ET}}{ }^{*}$ is a convex function of $c_{p}$. Further, solving $\pi_{p}^{\mathrm{EB}}-\pi_{p}^{\mathrm{ET}}=0$, we obtain that there exists two roots (with respect to $c_{p}$ ):

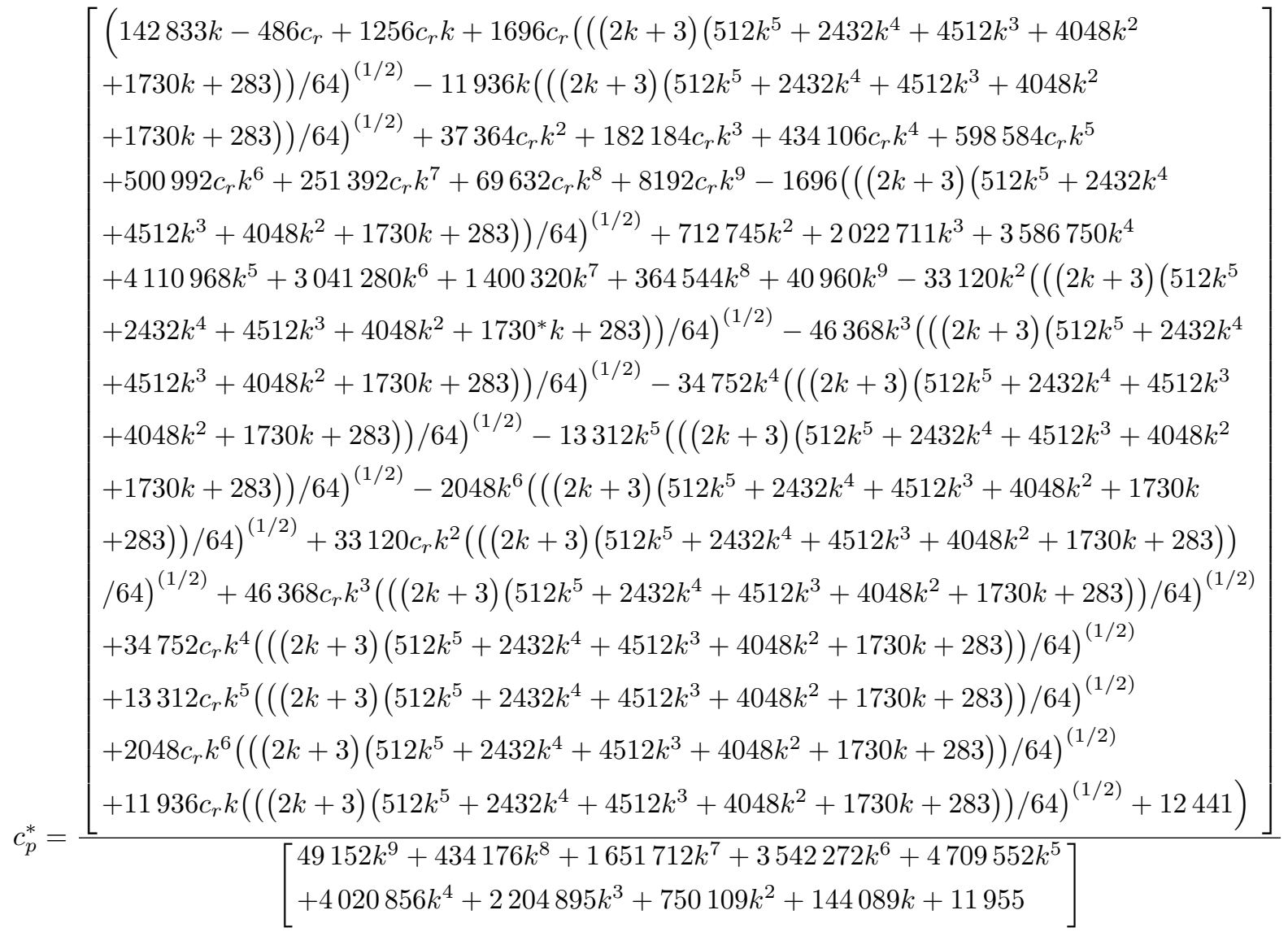


and

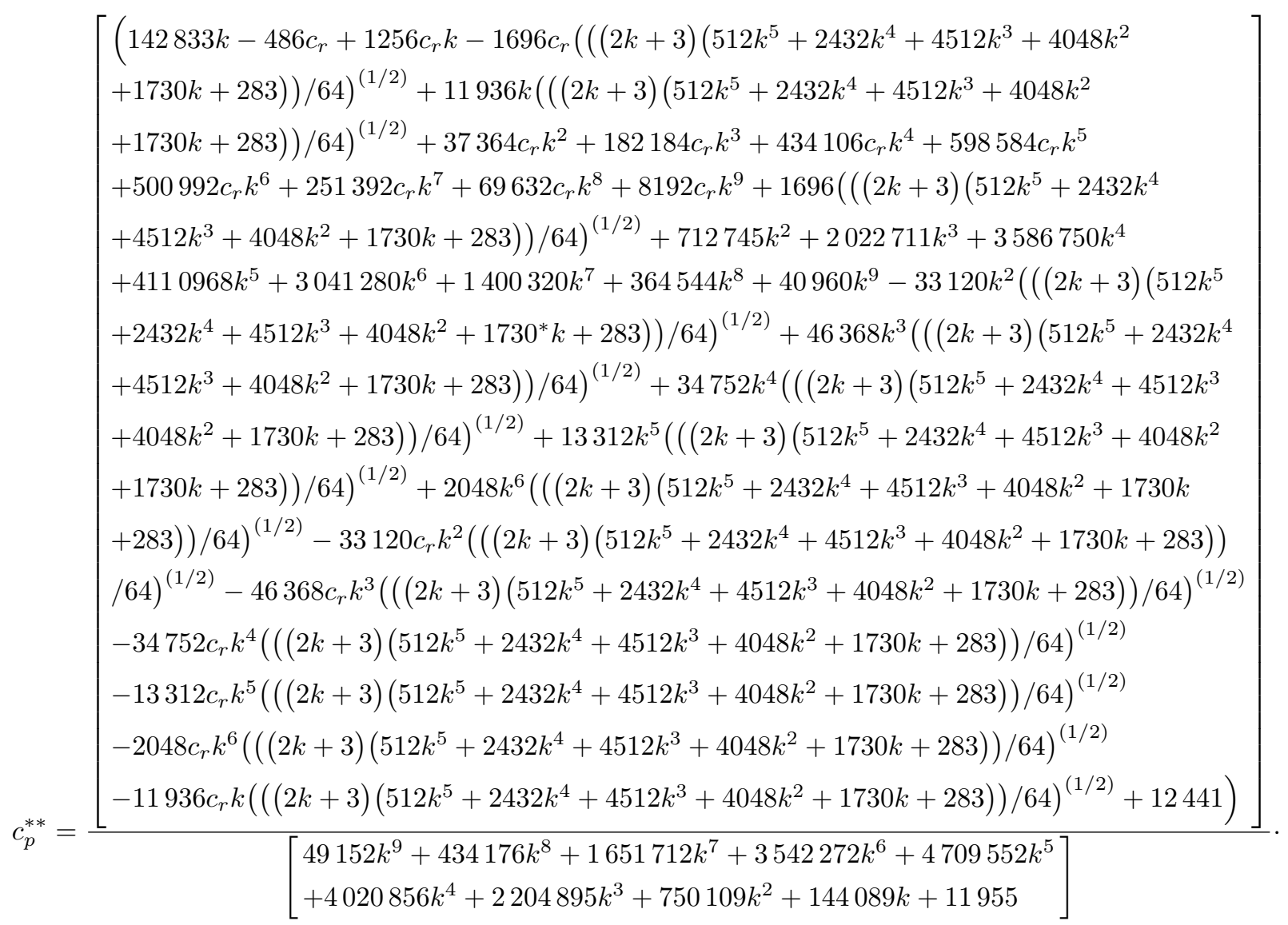

To simplify the comparison, we can obtain that, $\underline{\underline{c_{p}}}<\overline{\overline{c_{p}}}<c_{p}^{*}<c_{p}^{* *}$. Thus, we can conclude that, for any $c_{p}$, $\pi_{p}^{\mathrm{EB}^{*}}-\pi_{p}^{\mathrm{ET}}>0$ always holds.

Lemma A.1. In Model EM under simultaneous setting, we have:

(i) The equilibrium wholesale prices, quantities, and commission fee are:

$$
\begin{aligned}
w_{r}^{\mathrm{EM}}{ }^{*} & =\frac{-2 c_{p}-17 c_{r}+12 k-12 c_{r} k+19}{28 k+41} \\
f_{p}^{\mathrm{EM}^{*}} & =\frac{20 c_{p}+6 c_{r}+20 k+8 c_{r} k+15}{28 k+41} \\
q_{s}^{\mathrm{EM}}{ }^{*} & =\frac{4 c_{r}-14 c_{p}+10}{28 k+41} \\
q_{r}^{\mathrm{EM}} & =\frac{8 c_{p}-14 c_{r}+8 k-8 c_{r} k+6}{28 k+41} .
\end{aligned}
$$

(ii) The equilibrium profits of the supplier, traditional retailer, and platform owner are:

$$
\pi_{s}^{\mathrm{EM}^{*}}=\frac{\left[\begin{array}{l}
180 c_{p}^{2}-80 c_{p} c_{r} k-220 c_{p} c_{r}+80 c_{p} k-140 c_{p}+96 c_{r}^{2} k^{2}+304 c_{r}^{2} k \\
+254 c_{r}^{2}-192 c_{r} k^{2}-528 c_{r} k-288 c_{r}+96 k^{2}+224 k+214
\end{array}\right]}{(28 k+41)^{2}}
$$




$$
\begin{aligned}
\pi_{r}^{\mathrm{EM}^{*}} & =\frac{4\left(4 c_{p}-7 c_{r}+4 k-4 c_{r} k+3\right)^{2}}{(28 k+41)^{2}} \\
\pi_{p}^{\mathrm{EM}^{*}} & =\frac{2(2 k+3)\left(2 c_{r}-7 c_{p}+5\right)^{2}}{(28 k+41)^{2}} .
\end{aligned}
$$

Proof of Lemma A.1. Based on the timeline in the Model EM under simultaneous setting, solving the first-order conditions of the supplier's profit from equation (3.4) with respect to $q_{s}^{\mathrm{EM}}$, and the traditional retailer's profit from equation (3.5) with respect to $q_{r}^{\mathrm{EM}}$ yield $q_{s}^{\mathrm{EM}}{ }^{*}=\frac{c_{r}-2 f^{\mathrm{EM}}+w_{r}^{\mathrm{EM}}+1}{3}$ and $q_{r}^{\mathrm{EM}}{ }^{*}=\frac{f^{\mathrm{EM}}-2 c_{r}-2 w_{r}^{\mathrm{EM}}+1}{3}$.

After substituting $q_{s}^{\mathrm{EM}^{*}}\left(w_{r}^{\mathrm{EM}}, f^{\mathrm{EM}}\right)$ and $q_{r}^{\mathrm{EM}^{*}}\left(w_{r}^{\mathrm{EM}}, f^{\mathrm{EM}}\right)$ into equations (3.4) and (3.6), the supplier and platform owner's profits are given respectively: $\pi_{s}^{\mathrm{EM}}{ }^{*}=\left(2 c_{r}-4 f^{\mathrm{EM}}+5 w_{r}^{\mathrm{EM}}-4 c_{r} f^{\mathrm{EM}}-4 c_{r} w_{r}^{\mathrm{EM}}-f^{\mathrm{EM}} w_{r}^{\mathrm{EM}}+\right.$ $\left.c_{r}^{2}+4 f^{\mathrm{EM}^{2}}-5 w_{r}^{\mathrm{EM}^{2}}+1\right) / 9$ and $\pi_{p}^{\mathrm{EM}}{ }^{*}=\left(-c_{r}+2 f^{\mathrm{EM}}-w_{r}^{\mathrm{EM}}-1\right)\left(3 c_{p}-3 f^{\mathrm{EM}}+k+c_{r} k-2 f^{\mathrm{EM}} k+k w_{r}^{\mathrm{EM}}\right) / 9$. Then, solving the first-order conditions of the supplier's profit with respect to $w_{r}^{\mathrm{EM}}$, and the platform owner's profit with respect to $f^{\mathrm{EM}}$ yield $w_{r}^{\mathrm{EM}^{*}}=\frac{-2 c_{p}-17 c_{r}+12 k-12 c_{r} k+19}{28 k+41}$ and $f^{\mathrm{EM}^{*}}=\frac{20 c_{p}+6 c_{r}+20 k+8 c_{r} k+15}{28 k+41}$.

Furthermore, substituting the optimal wholesale prices $w_{r}^{\mathrm{EM}^{*}}$ and $f^{\mathrm{EM}^{*}}$ into $q_{s}^{\mathrm{EM}}\left(w_{r}^{\mathrm{EM}}, f^{\mathrm{EM}}\right)$, and $q_{r}^{\mathrm{EM}^{*}}\left(w_{r}^{\mathrm{EM}}, f^{\mathrm{EM}}\right)$, we derive the optimal quantities. That is, $q_{s}^{\mathrm{EM}^{*}}=\frac{4 c_{r}-14 c_{p}+10}{28 k+41}$ and $q_{r}^{\mathrm{EM}^{*}}=\frac{8 c_{p}-14 c_{r}+8 k-8 c_{r} k+6}{28 k+41}$.

Substituting the optimal $w_{r}^{\mathrm{EM}^{*}}, f^{\mathrm{EM}^{*}}, q_{r}^{\mathrm{EM}^{*}}$ and $q_{s}^{\mathrm{EM}^{*}}$ into equations (3.4), (3.5) and (3.6), we have the profits of all players as follow:

$$
\begin{aligned}
\pi_{s}^{\mathrm{EM}^{*}} & =\frac{\left[\begin{array}{l}
180 c_{p}^{2}-80 c_{p} c_{r} k-220 c_{p} c_{r}+80 c_{p} k-140 c_{p}+96 c_{r}^{2} k^{2}+304 c_{r}^{2} k \\
+254 c_{r}^{2}-192 c_{r} k^{2}-528 c_{r} k-288 c_{r}+96 k^{2}+224 k+214
\end{array}\right]}{(28 k+41)^{2}}, \\
\pi_{p}^{\mathrm{EM}} & =\frac{2(2 k+3)\left(2 c_{r}-7 c_{p}+5\right)^{2}}{(28 k+41)^{2}} \text { and } \pi_{r}^{\mathrm{EM}^{*}}=\frac{4\left(4 c_{p}-7 c_{r}+4 k-4 c_{r} k+3\right)^{2}}{(28 k+41)^{2}} .
\end{aligned}
$$

Lemma A.2. In Model ET under simultaneous setting, we have:

(i) The equilibrium wholesale prices and quantities are:

$$
\begin{aligned}
w_{r}^{\mathrm{ET}} & =\frac{1-c_{r}}{2} \\
w_{P}^{\mathrm{ET}^{*}} & =\frac{1-c_{p}}{2} \\
q_{r}^{\mathrm{ET}} & =\frac{c_{p}-2 c_{r}+2 k-2 c_{r} k+1}{8 k+6} \\
q_{p}^{\mathrm{ET}^{*}} & =\frac{c_{r}-2 c_{p}+1}{8 k+6} .
\end{aligned}
$$

(ii) The equilibrium profits of the supplier, traditional retailer, and platform owner are:

$$
\begin{aligned}
\pi_{s}^{\mathrm{ET}} & =\frac{k-c_{r}-c_{p}-c_{p} c_{r}-2 c_{r} k+c_{r}^{2} k+c_{p}^{2}+c_{r}^{2}+1}{8 k+6} \\
\pi_{r}^{\mathrm{ET}} & =\frac{\left(c_{p}-2 c_{r}+2 k-2 c_{r} k+1\right)^{2}}{(8 k+6)^{2}} \\
\pi_{p}^{\mathrm{ET}} & =\frac{(k+1)\left(c_{r}-2 c_{p}+1\right)^{2}}{4(4 k+3)^{2}} .
\end{aligned}
$$


Proof of Lemma A.2. Based on the timeline in the Model ET under simultaneous setting, solving the first-order conditions of the traditional retailer's profit from equation (3.11) with respect to $q_{r}^{\mathrm{ET}}$, and the platform owner's profit from equation (3.12) with respect to $q_{p}^{\mathrm{ET}}$ yield $q_{r}^{\mathrm{ET}}=\left(c_{p}-2 c_{r}+2 k+w_{p}^{\mathrm{ET}}-2 w_{r}^{\mathrm{ET}}-2 c_{r} k-2 k w_{r}^{\mathrm{ET}}+\right.$ $1) /(4 k+3)$ and $q_{p}^{\mathrm{ET}^{*}}=\left(c_{r}-2 c_{p}-2 w_{p}^{\mathrm{ET}}+w_{r}^{\mathrm{ET}}+1\right) /(4 k+3)$.

After substituting $q_{r}^{\mathrm{ET}}\left(w_{r}^{\mathrm{ET}}, w_{p}^{\mathrm{ET}}\right)$ and $q_{p}^{\mathrm{ET}}\left(w_{r}^{\mathrm{ET}}, w_{p}^{\mathrm{ET}}\right)$ into equation (3.10), the supplier's profits are given:

$$
\pi_{s}^{\mathrm{ET}}=\frac{\left[\begin{array}{l}
w_{p}^{\mathrm{ET}}+w_{r}^{\mathrm{ET}}-2 c_{p} w_{p}^{\mathrm{ET}}+c_{p} w_{r}^{\mathrm{ET}}+c_{r} w_{p}^{\mathrm{ET}}-2 c_{r} w_{r}^{\mathrm{ET}}+2 k w_{r}^{\mathrm{ET}} \\
+2 w_{p}^{\mathrm{ET}} w_{r}^{\mathrm{ET}}-2 k w_{r}^{\mathrm{ET}}-2 w_{p}^{\mathrm{ET}}-2 w_{r}^{\mathrm{ET}}-2 c_{r} k w_{r}^{\mathrm{ET}}
\end{array}\right]}{4 k+3} .
$$

Then, solving the first-order conditions of the supplier's profit with respect to $w_{r}^{\mathrm{ET}}$ and $w_{p}^{\mathrm{ET}}$ yield: $w_{r}^{\mathrm{ET}^{*}}=\frac{1-c_{r}}{2}$ and $w_{p}^{\mathrm{ET}}=\frac{1-c_{p}}{2}$. Furthermore, substituting the optimal wholesale prices $w_{r}^{\mathrm{ET}^{*}}$ and $w_{P}^{\mathrm{ET}^{*}}$ into $q_{p}^{\mathrm{ET}}{ }^{*}\left(w_{r}^{\mathrm{ET}}, w_{p}^{\mathrm{ET}}\right)$ and $q_{r}^{\mathrm{ET}^{*}}\left(w_{r}^{\mathrm{ET}}, w_{p}^{\mathrm{ET}}\right)$, we can derive the optimal quantities. That is, $q_{p}^{\mathrm{ET}^{*}}=\left(c_{r}-2 c_{p}+1\right) /(8 k+6)$ and $q_{r}^{\mathrm{ET}^{*}}=\left(c_{p}-2 c_{r}+2 k-2 c_{r} k+1\right) /(8 k+6)$. Substituting the optimal $w_{r}^{\mathrm{ET}^{*}}, w_{P}^{\mathrm{ET}^{*}}, q_{p}^{\mathrm{ET}^{*}}$ and $q_{r}^{\mathrm{ET}^{*}}$ into equations (3.10), (3.11) and (3.12), we have the profits of all players as follow:

$$
\begin{aligned}
& \pi_{s}^{\mathrm{ET}^{*}}=\left(k-c_{r}-c_{p}-c_{p} c_{r}-2 c_{r} k+c_{r}^{2} k+c_{p}^{2}+c_{r}^{2}+1\right) /(8 k+6), \\
& \pi_{r}^{\mathrm{ET}^{*}}=\left(c_{p}-2 c_{r}+2 k-2 c_{r} k+1\right)^{2} /(8 k+6)^{2} \quad \text { and } \quad \pi_{p}^{\mathrm{ET}^{*}}=(k+1)\left(c_{r}-2 c_{p}+1\right)^{2} /\left(4(4 k+3)^{2}\right) .
\end{aligned}
$$

Lemma A.3. In Model EB under simultaneous setting, we have:

(i) The equilibrium wholesale prices, quantities, and commission fee are:

$$
\begin{aligned}
w_{r}^{\mathrm{EB}} & =\frac{75 k-30 c_{r}+8 c_{p} k-83 c_{r} k+8 c_{p} k^{2}-68 c_{r} k^{2}-16 c_{r} k^{3}+60 k^{2}+16 k^{3}+30}{2\left(16 k^{3}+70 k^{2}+89 k+34\right)} \\
w_{p}^{\mathrm{EB}} & =\frac{4 c_{r}-34 c_{p}+61 k-64 c_{p} k+3 c_{r} k-32 c_{p} k^{2}+32 k^{2}+30}{2\left(16 k^{3}+70 k^{2}+89 k+34\right)} \\
f_{p}^{\mathrm{EB}} & =\frac{17 c_{p}+5 c_{r}+41 k+29 c_{p} k+19 c_{r} k+14 c_{p} k^{2}+17 c_{r} k^{2}+4 c_{r} k^{3}+39 k^{2}+12 k^{3}+12}{2\left(16 k^{3}+70 k^{2}+89 k+34\right)} \\
q_{s}^{\mathrm{EB}} & =\frac{3 c_{r}-17 c_{p}+28 k-30 c_{p} k+2 c_{r} k-16 c_{p} k^{2}+16 k^{2}+14}{32 k^{3}+140 k^{2}+178 k+68} \\
q_{P}^{\mathrm{EB}} & =\frac{9 c_{r}-17 c_{p}+5 k-18 c_{p} k+13 c_{r} k+4 c_{r} k^{2}-4 k^{2}+8}{32 k^{3}+140 k^{2}+178 k+68} \\
q_{r}^{\mathrm{EB}} & =\frac{17 c_{p}-25 c_{r}+35 k+20 c_{p} k-55 c_{r} k+4 c_{p} k^{2}-38 c_{r} k^{2}-8 c_{r} k^{3}+34 k^{2}+8 k^{3}+8}{32 k^{3}+140 k^{2}+178 k+68} .
\end{aligned}
$$

(ii) The equilibrium profits of the supplier, traditional retailer, and platform owner are:

$$
\begin{aligned}
& {\left[288 c_{p}^{2} k^{4}+1728 c_{p}^{2} k^{3}+3436 c_{p}^{2} k^{2}+2856 c_{p}^{2} k+867 c_{p}^{2}-128 c_{p} c_{r} k^{5}-1088 c_{p} c_{r} k^{4}\right]} \\
& -3444 c_{p} c_{r} k^{3}-5102 c_{p} c_{r} k^{2}-3600 c_{p} c_{r} k-986 c_{p} c_{r}+128 c_{p} k^{5}+512 c_{p} k^{4} \\
& -12 c_{p} k^{3}-1770 c_{p} k^{2}-2112 c_{p} k-748 c_{p}+128 c_{r}^{2} k^{6}+1152 c_{r}^{2} k^{5}+4128 c_{r}^{2} k^{4} \\
& +7546 c_{r}^{2} k^{3}+7464 c_{r}^{2} k^{2}+3816 c_{r}^{2} k+795 c_{r}^{2}-256 c_{r} k^{6}-2176 c_{r} k^{5}-7168 c_{r} k^{4} \\
& -11648 c_{r} k^{3}-9826 c_{r} k^{2}-4032 c_{r} k-604 c_{r}+128 k^{6}+1024 k^{5}+3328 k^{4} \\
& l \pi_{s}^{\mathrm{EB}^{*}}=\frac{\left[+5830 k^{3}+5798 k^{2}+3072 k+676\right.}{4\left(16 k^{3}+70 k^{2}+89 k+34\right)^{2}} \\
& \pi_{r}^{\mathrm{EB}^{*}}=\frac{\left(17 c_{p}-25 c_{r}+35 k+20 c_{p} k-55 c_{r} k+4 c_{p} k^{2}-38 c_{r} k^{2}-8 c_{r} k^{3}+34 k^{2}+8 k^{3}+8\right)^{2}}{4\left(16 k^{3}+70 k^{2}+89 k+34\right)^{2}}
\end{aligned}
$$




$$
\pi_{p}^{\mathrm{EB}^{*}}=\frac{\left[\begin{array}{l}
256 c_{p}^{2} k^{5}+1792 c_{p}^{2} k^{4}+4704 c_{p}^{2} k^{3}+5964 c_{p}^{2} k^{2}+3672 c_{p}^{2} k+867 c_{p}^{2}-128 c_{p} c_{r} k^{5} \\
-928 c_{p} c_{r} k^{4}-2616 c_{p} c_{r} k^{3}-3576 c_{p} c_{r} k^{2}-2344 c_{p} c_{r} k-578 c_{p} c_{r}-384 c_{p} k^{5} \\
-2656 c_{p} k^{4}-6792 c_{p} k^{3}-8352 c_{p} k^{2}-5000 c_{p} k-1156 c_{p}+16 c_{r}^{2} k^{5}+136 c_{r}^{2} k^{4} \\
+433 c_{r}^{2} k^{3}+641 c_{r}^{2} k^{2}+440 c_{r}^{2} k+111 c_{r}^{2}+96 c_{r} k^{5}+656 c_{r} k^{4}+1750 c_{r} k^{3}+400 \\
+2294 c_{r} k^{2}+1464 c_{r} k+356 c_{r}+144 k^{5}+1000 k^{4}+2521 k^{3}+3029 k^{2}+1768 k
\end{array}\right]}{4\left(16 k^{3}+70 k^{2}+89 k+34\right)^{2}} .
$$

Proof of Lemma A.3. Based on the timeline in the Model EB under simultaneous setting, solving the first-order conditions of the supplier's profit from equation (3.15) with respect to $q_{s}^{\mathrm{EB}}$, the platform owner's profit from equation (3.16) with respect to $q_{r}^{\mathrm{EB}}$ and the traditional retailer's profit from equation (3.17) with respect to $q_{p}^{\mathrm{EB}}$ yield $q_{s}^{\mathrm{EB}}=\frac{c_{p}+c_{r}-3 f^{\mathrm{EB}}+2 k+w_{p}^{\mathrm{EB}}+w_{r}^{\mathrm{EB}}+2 c_{r} k-4 f^{\mathrm{EB}} k+2 k w_{r}^{\mathrm{EB}}+1}{4 k+4}, q_{r}^{\mathrm{EB}}=\frac{c_{p}-3 c_{r}+f^{\mathrm{EB}}+2 k+w_{p}^{\mathrm{EB}}-3 w_{r}^{\mathrm{EB}}-2 c_{r} k-2 k w_{r}^{\mathrm{EB}}+1}{4 k+4}$ and $q_{P}^{\mathrm{EB}}=\frac{c_{r}-3 c_{p}+f^{\mathrm{EB}}-2 k-3 w_{p}^{\mathrm{EB}}+w_{r}^{\mathrm{EB}}-2 c_{r} k+4 f^{\mathrm{EB}} k-2 \mathrm{k} w_{r}^{\mathrm{EB}}+1}{4 k+4}$.

After substituting $q_{s}^{\mathrm{EB}}\left(w_{p}^{\mathrm{EB}}, w_{r}^{\mathrm{EB}}, f^{\mathrm{EB}}\right), q_{P}^{\mathrm{EB}}\left(w_{p}^{\mathrm{EB}}, w_{r}^{\mathrm{EB}}, f^{\mathrm{EB}}\right)$ and $q_{r}^{\mathrm{EB}}\left(w_{p}^{\mathrm{EB}}, w_{r}^{\mathrm{EB}}, f^{\mathrm{EB}}\right)$ into equations (3.15) and (3.16), the supplier and platform owner's profits are given respectively:

$$
\pi_{s}^{\mathrm{EB}}=\frac{\left[\begin{array}{l}
c_{p}^{2}+4 c_{p} c_{r} k+2 c_{p} c_{r}-8 c_{p} f^{\mathrm{EB}} k-6 c_{p} f^{\mathrm{EB}}-12 c_{p} k w_{p}^{\mathrm{EB}}+8 c_{p} k w_{r}^{\mathrm{EB}}+4 c_{p} k-10 c_{p} w_{p}^{\mathrm{EB}}+6 c_{p} w_{r}^{\mathrm{EB}} \\
+2 c_{p}+4 c_{r}^{2} k^{2}+4 c_{r}^{2} k+c_{r}^{2}-16 c_{r} f^{\mathrm{EB}} k^{2}-20 c_{r} f^{\mathrm{EB}} k-6 c_{r} f^{\mathrm{EB}}-8 c_{r} k^{2} w_{p}^{\mathrm{EB}} 8 c_{r} k^{2}-12 c_{r} k w_{r}^{\mathrm{EB}} \\
+8 c_{r} k+6 c_{r} w_{p}^{\mathrm{EB}}-10 c_{r} w_{r}^{\mathrm{EB}}+2 c_{r}+16 f^{\mathrm{EB}} k^{2}+24 f^{\mathrm{EB}} k+9 f^{\mathrm{EB}}{ }^{2}+16 f^{\mathrm{EB}} k^{2} w_{p}^{\mathrm{EB}}-16 f^{\mathrm{EB}} k^{2} w_{r}^{\mathrm{EB}} \\
-16 f^{\mathrm{EB}} k^{2}+12 f^{\mathrm{EB}} k w_{p}^{\mathrm{EB}}-16 f^{\mathrm{EB}} k w_{r}^{\mathrm{EB}}-20 f^{\mathrm{EB}} k-2 f^{\mathrm{EB}} w_{p}^{\mathrm{EB}}-2 f^{\mathrm{EB}} w_{r}^{\mathrm{EB}}-6 f^{\mathrm{EB}}-8 k^{2} w_{p}^{\mathrm{EB}} w_{r}^{\mathrm{EB}} \\
-8 k^{2} w_{p}^{\mathrm{EB}}-4 k^{2} w_{r}^{\mathrm{EB}}+16 k^{2} w_{r}^{\mathrm{EB}}+4 k^{2}-12 k w_{p}^{2}+4 k w_{p}^{\mathrm{EB}} w_{r}^{\mathrm{EB}}-16 k w_{r}^{\mathrm{EB}}+20 k w_{r}^{\mathrm{EB}} \\
+4 k-11 w_{p}^{\mathrm{EB}}+10 w_{p}^{\mathrm{EB}} w_{r}^{\mathrm{EB}}+6 w_{p}^{\mathrm{EB}}-11 w_{r}^{\mathrm{EB}}+6 w_{r}^{\mathrm{EB}}+1
\end{array}\right]}{16(k+1)^{2}}
$$

and

$$
\pi_{p}^{\mathrm{EB}}=\frac{\left[\begin{array}{l}
4 c_{p}^{2} k+5 c_{p}^{2}-8 c_{p} c_{r} k-10 c_{p} c_{r}+8 c_{p} f^{\mathrm{EB}} k+10 c_{p} f^{\mathrm{EB}}+12 c_{p} k w_{p}^{\mathrm{EB}}-8 c_{p} k w_{r}^{\mathrm{EB}}-8 c_{p} k+14 c_{p} w_{p}^{\mathrm{EB}} \\
-10 c_{p} w_{r}^{\mathrm{EB}}-10 c_{p}-4 c_{r}^{2} k^{2}-4 c_{r}^{2} k+c_{r}^{2}+16 c_{r} f^{\mathrm{EB}} k^{2}+24 c_{r} f^{\mathrm{EB}} k+6 c_{r} f^{\mathrm{EB}}+8 c_{r} k^{2} w_{p}^{\mathrm{EB}}-8 c_{r} k^{2} w_{r}^{\mathrm{EB}} \\
-8 c_{r} k^{2}+4 c_{r} k w_{p}^{\mathrm{EB}}-8 c_{r} k w_{r}^{\mathrm{EB}}-8 c_{r} k-6 c_{r} w_{p}^{\mathrm{EB}}+2 c_{r} w_{r}^{\mathrm{EB}}+2 c_{r}-16 f^{\mathrm{EB}} k^{2}-28 f^{\mathrm{EB}} k-11 f^{\mathrm{EB}^{2}} \\
-16 f^{\mathrm{EB}} k^{2} w_{p}^{\mathrm{EB}}+16 f^{\mathrm{EB}} k^{2} w_{r}^{\mathrm{EB}}+16 f^{\mathrm{EB}} k^{2}-20 f^{\mathrm{EB}} k w_{p}^{\mathrm{EB}}+24 f^{\mathrm{EB}} k w_{r}^{\mathrm{EB}}+24 f^{\mathrm{EB}} k-2 f^{\mathrm{EB}} w_{p}^{\mathrm{EB}} \\
+6 f^{\mathrm{EB}} w_{r}^{\mathrm{EB}}+6 f^{\mathrm{EB}}+8 k^{2} w_{p}^{\mathrm{EB}} w_{r}^{\mathrm{EB}}+8 k^{2} w_{p}^{\mathrm{EB}}-4 k^{2} w_{r}^{\mathrm{EB}}-8 k^{2} w_{r}^{\mathrm{EB}}-4 k^{2}+8 k w_{p}^{2}+4 k w_{p}^{\mathrm{EB}} w_{r}^{\mathrm{EB}} \\
+4 k w_{p}^{\mathrm{EB}}-4 k w_{r}^{\mathrm{EB}}-8 k w_{r}^{\mathrm{EB}}-4 k+9 w_{p}^{\mathrm{EB}}-6 w_{p}^{\mathrm{EB}} w_{r}^{\mathrm{EB}}-6 w_{p}^{\mathrm{EB}}+w_{r}^{\mathrm{EB}}+2 w_{r}^{\mathrm{EB}}+1 \\
16(k+1)^{2}
\end{array}\right]}{.}
$$

Then, solving the first-order conditions of the supplier's profit with respect to $w_{r}^{\mathrm{EB}}$ and $w_{p}^{\mathrm{EB}}$, and the platform owner's profit with respect to $f^{\mathrm{EB}}$ yield:

$$
\begin{aligned}
w_{r}^{\mathrm{EB}} & =\frac{75 k-30 c_{r}+8 c_{p} k-83 c_{r} k+8 c_{p} k^{2}-68 c_{r} k^{2}-16 c_{r} k^{3}+60 k^{2}+16 k^{3}+30}{2\left(16 k^{3}+70 k^{2}+89 k+34\right)}, \\
w_{p}^{\mathrm{EB}} & =\frac{4 c_{r}-34 c_{p}+61 k-64 c_{p} k+3 c_{r} k-32 c_{p} k^{2}+32 k^{2}+30}{2\left(16 k^{3}+70 k^{2}+89 k+34\right)} \text { and } \\
f_{p}^{\mathrm{EB}} & =\frac{17 c_{p}+5 c_{r}+41 k+29 c_{p} k+19 c_{r} k+14 c_{p} k^{2}+17 c_{r} k^{2}+4 c_{r} k^{3}+39 k^{2}+12 k^{3}+12}{2\left(16 k^{3}+70 k^{2}+89 k+34\right)} .
\end{aligned}
$$

Furthermore, substituting the optimal wholesale prices $w_{r}^{\mathrm{EB}}, w_{P}^{\mathrm{EB}}$ and $f^{\mathrm{EB}^{*}}$ into $q_{s}^{\mathrm{EB}}{ }^{*}\left(w_{p}^{\mathrm{EB}}, w_{r}^{\mathrm{EB}}, f^{\mathrm{EB}}\right)$, $q_{P}^{\mathrm{EB}}{ }^{*}\left(w_{p}^{\mathrm{EB}}, w_{r}^{\mathrm{EB}}, f^{\mathrm{EB}}\right)$ and $q_{r}^{\mathrm{EB}}\left(w_{p}^{\mathrm{EB}}, w_{r}^{\mathrm{EB}}, f^{\mathrm{EB}}\right)$, we can derive the optimal quantities. That is,

$$
q_{s}^{\mathrm{EB}}=\frac{3 c_{r}-17 c_{p}+28 k-30 c_{p} k+2 c_{r} k-16 c_{p} k^{2}+16 k^{2}+14}{32 k^{3}+140 k^{2}+178 k+68},
$$




$$
\begin{aligned}
q_{P}^{\mathrm{EB}} & =\frac{9 c_{r}-17 c_{p}+5 k-18 c_{p} k+13 c_{r} k+4 c_{r} k^{2}-4 k^{2}+8}{32 k^{3}+140 k^{2}+178 k+68} \text { and } \\
q_{r}^{\mathrm{EB}} & =\frac{17 c_{p}-25 c_{r}+35 k+20 c_{p} k-55 c_{r} k+4 c_{p} k^{2}-38 c_{r} k^{2}-8 c_{r} k^{3}+34 k^{2}+8 k^{3}+8}{32 k^{3}+140 k^{2}+178 k+68} .
\end{aligned}
$$

Substituting the optimal $w_{r}^{\mathrm{EB}}, w_{P}^{\mathrm{EB}^{*}}, f^{\mathrm{EB}^{*}}, q_{r}^{\mathrm{EB}^{*}}, q_{P}^{\mathrm{EB}^{*}}$ and $q_{s}^{\mathrm{EB}^{*}}$ into equations (3.15), (3.16) and (3.17), we have the profits of all players as follow:

$$
\begin{aligned}
& \pi_{s}^{\mathrm{EB}}=\frac{\left[\begin{array}{l}
288 c_{p}^{2} k^{4}+1728 c_{p}^{2} k^{3}+3436 c_{p}^{2} k^{2}+2856 c_{p}^{2} k+867 c_{p}^{2}-128 c_{p} c_{r} k^{5}-1088 c_{p} c_{r} k^{4}-3444 c_{p} c_{r} k^{3} \\
-5102 c_{p} c_{r} k^{2}-3600 c_{p} c_{r} k-986 c_{p} c_{r}+128 c_{p} k^{5}+512 c_{p} k^{4}-12 c_{p} k^{3}-1770 c_{p} k^{2}-2112 c_{p} k \\
-748 c_{p}+128 c_{r}^{2} k^{6}+1152 c_{r}^{2} k^{5}+4128 c_{r}^{2} k^{4}+7546 c_{r}^{2} k^{3}+7464 c_{r}^{2} k^{2}+3816 c_{r}^{2} k+795 c_{r}^{2} \\
-256 c_{r} k^{6}-2176 c_{r} k^{5}-7168 c_{r} k^{4}-11648 c_{r} k^{3}-9826 c_{r} k^{2}-4032 c_{r} k-604 c_{r}+128 k^{6} \\
+1024 k^{5}+3328 k^{4}+5830 k^{3}+5798 k^{2}+3072 k+676
\end{array}\right]}{4\left(16 k^{3}+70 k^{2}+89 k+34\right)^{2}}, \\
& \pi_{p}^{\mathrm{EB}^{*}}=\frac{\left[\begin{array}{l}
256 c_{p}^{2} k^{5}+1792 c_{p}^{2} k^{4}+4704 c_{p}^{2} k^{3}+5964 c_{p}^{2} k^{2}+3672 c_{p}^{2} k+867 c_{p}^{2}-128 c_{p} c_{r} k^{5}-928 c_{p} c_{r} k^{4} \\
-2616 c_{p} c_{r} k^{3}-3576 c_{p} c_{r} k^{2}-2344 c_{p} c_{r} k-578 c_{p} c_{r}-384 c_{p} k^{5}-2656 c_{p} k^{4}-6792 c_{p} k^{3} \\
-8352 c_{p} k^{2}-5000 c_{p} k-1156 c_{p}+16 c_{r}^{2} k^{5}+136 c_{r}^{2} k^{4}+433 c_{r}^{2} k^{3}+641 c_{r}^{2} k^{2}+440 c_{r}^{2} k+111 c_{r}^{2} \\
+96 c_{r} k^{5}+656 c_{r} k^{4}+1750 c_{r} k^{3}+400+2294 c_{r} k^{2}+1464 c_{r} k+356 c_{r}+144 k^{5}+1000 k^{4} \\
+2521 k^{3}+3029 k^{2}+1768 k
\end{array}\right]}{4\left(16 k^{3}+70 k^{2}+89 k+34\right)^{2}} \\
& \pi_{r}^{\mathrm{EB}^{*}}=\frac{\left(17 c_{p}-25 c_{r}+35 k+20 c_{p} k-55 c_{r} k+4 c_{p} k^{2}-38 c_{r} k^{2}-8 c_{r} k^{3}+34 k^{2}+8 k^{3}+8\right)^{2}}{4\left(16 k^{3}+70 k^{2}+89 k+34\right)^{2}} .
\end{aligned}
$$

Next, we provide the proofs of propositions under simultaneous setting, similarly, all parameters and variables must satisfy non-negativity constraints. Hence, in the simultaneous setting, we only consider $c_{r} k+\frac{7}{4} c_{r}-k-\frac{3}{4}<$ $c_{p}<c_{r} \frac{2}{7}+\frac{5}{7}$ in the scenarios of Model EM (referred to as $c_{p}=c_{r} k+\frac{7}{4} c_{r}-k-\frac{3}{4}, \overline{c_{p}}=c_{r} \frac{2}{7}+\frac{5}{7}$ ), and $\frac{8 k^{3} c_{r}-8 k^{3}+38 k^{2} c_{r}-34 k^{2}+55 k c_{r}-35 k+25 c_{r}-8}{4 k^{2}+20 k+17}<c_{p}<\frac{4 k^{2} c_{r}-4 k^{2}+13 k c_{r}+\overline{5 k}+9 c_{r}+8}{18 k+17}$ in the scenarios of Model EB (referred to as $\left.c_{p}=\frac{8 k^{3} c_{r}-8 k^{3}+38 k^{2} c_{r}-34 k^{2}+55 k c_{r}-35 k+25 c_{r}-8}{4 k^{2}+20 k+17}, \overline{\overline{c_{p}}}=\frac{4 k^{2} c_{r}-4 k^{2}+13 k c_{r}+5 k+9 c_{r}+8}{18 k+17}\right)$. Under these constraints, we compare the outcomes among Model N, Model EM, Model ET and Model EB and draw the propositions in the simultaneous setting.

Proof of Proposition 5.1. Based on the outcomes of Lemmas 3.1 and A.1, we compare the supplier's profits between Model EM and Model N:

$$
\pi_{s}^{\mathrm{EM}^{*}}-\pi_{s}^{\mathrm{N}^{*}}=\frac{\left[\begin{array}{l}
1440 c_{p}^{2}-640 c_{p} c_{r} k-1760 c_{p} c_{r}+640 c_{p} k-1120 c_{p}-16 c_{r}^{2} k^{2}+136 c_{r}^{2} k \\
+351 c_{r}^{2}+32 c_{r} k^{2}+368 c_{r} k+1058 c_{r}-16 k^{2}-504 k+31
\end{array}\right]}{8(28 k+41)^{2}} .
$$

$\pi_{s}^{\mathrm{EM}^{*}}-\pi_{s}^{\mathrm{N}^{*}}$ is a quadratic function of $c_{p}$, and the coefficient $\frac{360}{(28 k+41)^{2}}$ is positive, which shows that the equation of $\pi_{s}^{\mathrm{EM}^{*}}-\pi_{s}^{\mathrm{N}^{*}}$ is a convex function of $c_{p}$. Further, solving $\pi_{s}^{\mathrm{EM}^{*}}-\pi_{s}^{\mathrm{N}^{*}}=0$, we obtain that there exist two roots (with respect to $c_{p}$ ):

$c_{p 4}=\left(11 c_{r}\right) / 18-(2 k) / 9+\left(41 \sqrt{10} c_{r}\right) / 360-(7 \sqrt{10} k) / 90+\left(2 c_{r} k\right) / 9-(41 \sqrt{10}) / 360+\left(7 \sqrt{10} c_{r} k\right) / 90+7 / 18$

and

$c_{p 4}^{*}=\left(11 c_{r}\right) / 18-(2 k) / 9-\left(41 \sqrt{10} c_{r}\right) / 360+(7 \sqrt{10} k) / 90+\left(2 c_{r} k\right) / 9+(41 \sqrt{10}) / 360-\left(7 \sqrt{10} c_{r} k\right) / 90+7 / 18$. 
This, together with the fact that $c_{p}<c_{p 4}<\overline{c_{p}}<c_{p 4}^{*}$, suggests that $\pi_{s}^{\mathrm{EM}^{*}}>\pi_{s}^{\mathrm{N}^{*}}$ when $c_{p}<c_{p 4}$. Otherwise, $\pi_{s}^{\mathrm{EM}^{*}}<\pi_{s}^{\mathrm{N}^{*}}$.

Similarly, comparing the traditional retailer's profits between Model EM and Model N, we can obtain:

$$
\pi_{r}^{\mathrm{EM}^{*}}-\pi_{r}^{\mathrm{N}^{*}}=\frac{\left[\begin{array}{l}
1024 c_{p}^{2}-2048 c_{p} c_{p} k-3584 c_{p} c_{p}+2048 c_{p} k+1536 c_{p}+240 c_{r}^{2} k^{2}+1288 c_{r}^{2} k \\
+1455 c_{r}^{2}-480 c_{p} k^{2}-528 c_{p} k+674 c_{p}+240 k^{2}-760 k-1105
\end{array}\right]}{16(28 k+41)^{2}} .
$$

$\pi_{r}^{\mathrm{EM}^{*}}-\pi_{r}^{\mathrm{N}^{*}}$ is also a quadratic function of $c_{p}$, and the coefficient $\frac{128}{(28 k+41)^{2}}$ is positive, which shows that the equation of $\pi_{r}^{\mathrm{EM}^{*}}-\pi_{r}^{\mathrm{N}^{*}}$ is a convex function of $c_{p}$. There are two roots (with respect to $c_{p}$ ) for the equation $\pi_{r}^{\mathrm{EM}^{*}}-\pi_{r}^{\mathrm{N}^{*}}=0: c_{p 5}=\frac{15 c_{r}}{32}-\frac{k}{8}+\frac{c_{r} k}{8}+\frac{17}{32}$ and $c_{p 5}^{*}=\frac{97 c_{r}}{32}-\frac{15 k}{8}+\frac{15 c_{r} k}{8}-\frac{65}{32}$.

After calculation, we can find $c_{p 5}^{*}<c_{p}<c_{p 5}<\overline{c_{p}}$. Thus, when $c_{p}>c_{p 5}$, we have $\pi_{r}^{\mathrm{EM}^{*}}>\pi_{r}^{\mathrm{N}^{*}}$; Otherwise $\pi_{r}^{\mathrm{EM}^{*}}<\pi_{r}^{\mathrm{N}^{*}}$. Furthermore, since $c_{p 4}<\bar{c}_{p 5}$, we can show that $\pi_{s}^{\mathrm{EM}^{*}}>\pi_{s}^{\mathrm{N}^{*}}$ and $\pi_{r}^{\mathrm{EM}^{*}}<\pi_{r}^{\mathrm{N}^{*}}$ when $c_{p}<c_{p 4}$; $\pi_{s}^{\mathrm{EM}^{*}}<\pi_{s}^{\mathrm{N}^{*}}$ and $\pi_{r}^{\mathrm{EM}^{*}}<\pi_{r}^{\mathrm{N}^{*}}$ when $c_{p 4}<c_{p}<c_{p 5} ; \pi_{s}^{\mathrm{EM}^{*}}<\pi_{s}^{\mathrm{N}^{*}}$ and $\pi_{r}^{\mathrm{EM}^{*}}>\pi_{r}^{\mathrm{N}^{*}}$ when $c_{p 5}<c_{p}$.

Proof of Proposition 5.2. Based on the outcomes of Lemmas 3.1 and A.3, we compare the supplier's profits between Model EB and Model N:

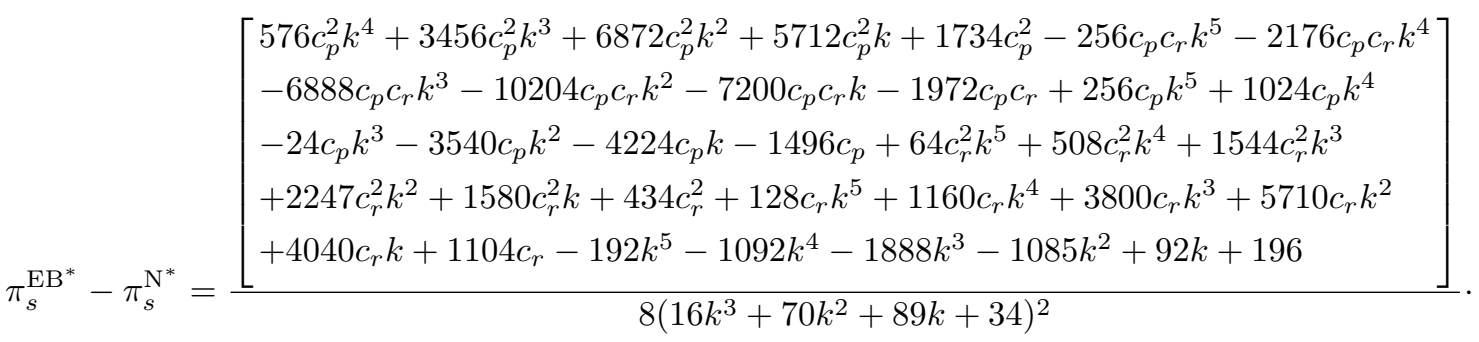

We find that $\pi_{s}^{\mathrm{EB}}-\pi_{s}^{\mathrm{N}^{*}}$ is a quadratic function of $c_{p}$, and the coefficient $\frac{576 k^{4}+3456 k^{3}+6872 k^{2}+5712 k+1734}{8\left(16 k^{3}+70 k^{2}+89 k+34\right)^{2}}$ is positive, which shows that the equation of $\pi_{s}^{\mathrm{EB}^{*}}-\pi_{s}^{\mathrm{N}^{*}}$ is a convex function of $c_{p}$. Further, solving $\pi_{s}^{\mathrm{EB}}-\pi_{s}^{\mathrm{N}^{*}}=0$, we obtain that there exist two roots (with respect to $c_{p}$ ):

$c_{p 6}=\frac{\left[\begin{array}{l}986 c_{r}+2112 k-34\left(2(4 k+5)\left(8 k^{3}+38 k^{2}+48 k+19\right)\right)^{\frac{1}{2}}+3600 c_{r} k+5102 c_{r} k^{2}+3444 c_{r} k^{3}+1088 c_{r} k^{4} \\ +128 c_{r} k^{5}+1770 k^{2}+12 k^{3}-512 k^{4}-128 k^{5}+34 c_{r}\left(2(4 k+5)\left(8 k^{3}+38 k^{2}+48 k+19\right)\right)^{\frac{1}{2}}-89 k(2(4 k \\ \left.+5)\left(8 k^{3}+38 k^{2}+48 k+19\right)\right)^{\frac{1}{2}}-70 k^{2}\left(2(4 k+5)\left(8 k^{3}+38 k^{2}+48 k+19\right)\right)^{\frac{1}{2}}-16 k^{3}\left(2(4 k+5)\left(8 k^{3}\right.\right. \\ \left.\left.+38 k^{2}+48 k+19\right)\right)^{\frac{1}{2}}+70 c_{r} k^{2}\left(2(4 k+5)\left(8 k^{3}+38 k^{2}+48 k+19\right)\right)^{\frac{1}{2}}+16 c_{r} k^{3}\left(2(4 k+5)\left(8 k^{3}+38 k^{2}\right.\right. \\ +48 k+19))^{\frac{1}{2}}+89 c_{r} k\left(2(4 k+5)\left(8 k^{3}+38 k^{2}+48 k+19\right)\right)^{\frac{1}{2}}+748\end{array}\right]}{2\left(288 k^{4}+1728 k^{3}+3436 k^{2}+2856 k+867\right)}$

and

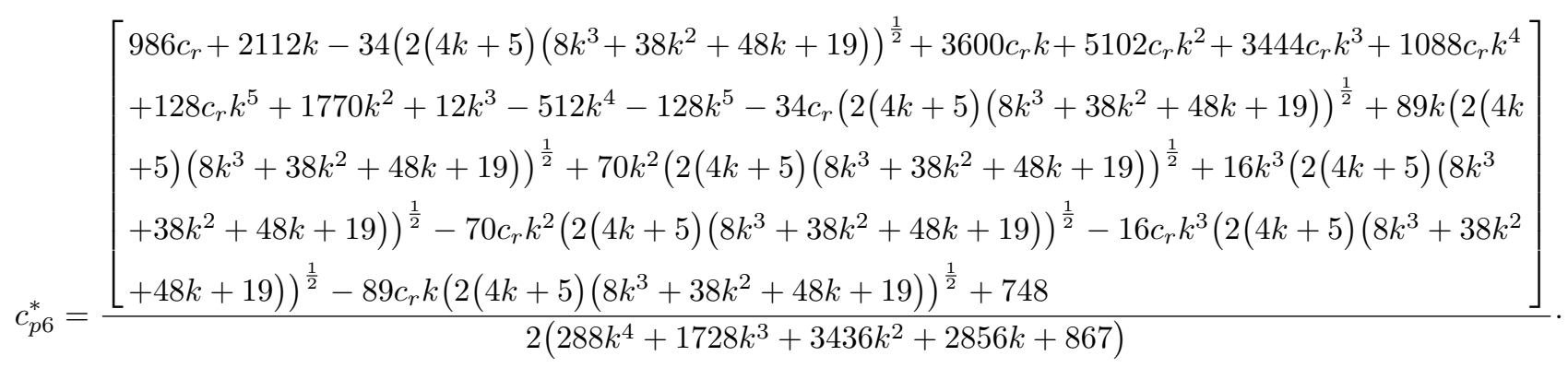


This, together with the fact that $\underline{c_{p}}<c_{p 6}<\overline{c_{p}}<c_{p 6}^{*}$, suggests that $\pi_{s}^{\mathrm{EB}}{ }^{*}>\pi_{s}^{\mathrm{N}^{*}}$ when $c_{p}<c_{p 6}$. Otherwise, $\pi_{s}^{\mathrm{EB}^{*}}<\pi_{s}^{\mathrm{N}^{*}}$.

Then, we compare the traditional retailer's profits between Model EB and Model N:

$$
\pi_{r}^{\mathrm{EB}^{*}}-\pi_{r}^{\mathrm{N}^{*}}=\frac{\left[\begin{array}{l}
64 c_{p}^{2} k^{4}+640 c_{p}^{2} k^{3}+2144 c_{p}^{2} k^{2}+2720 c_{p}^{2} k+1156 c_{p}^{2}-256 c_{p} c_{r} k^{5}-2496 c_{p} c_{r} k^{4} \\
-8928 c_{p} c_{r} k^{3}-14768 c_{p} c_{r} k^{2}-11480 c_{p} c_{r} k-3400 c_{p} c_{r}+256 c_{p} k^{5}+2368 c_{p} k^{4} \\
+7648 c_{p} k^{3}+10480 c_{p} k^{2}+6040 c_{p} k+1088 c_{p}+192 c_{r}^{2} k^{5}+1548 c_{r}^{2} k^{4}+4772 c_{r}^{2} k^{3} \\
+7019 c_{r}^{2} k^{2}+4948 c_{r}^{2} k+1344 c_{r}^{2}-128 c_{r} k^{5}-600 c_{r} k^{4}-616 c_{r} k^{3}+730 c_{r} k^{2} \\
+1584 c_{r} k+712 c_{r}-64 k^{5}-884 k^{4}-3516 k^{3}-5605 k^{2}-3812 k-900
\end{array}\right]}{16\left(16 k^{3}+70 k^{2}+89 k+34\right)^{2}} .
$$

$\pi_{r}^{\mathrm{EB}}-\pi_{r}^{\mathrm{N}^{*}}$ is a quadratic function of $c_{p}$, and the coefficient $\frac{\left(4 k^{2}+20 k+17\right)^{2}}{2\left(16 k^{3}+70 k^{2}+89 k+34\right)^{2}}$ is positive, which shows that the equation of $\pi_{r}^{\mathrm{EB}^{*}}-\pi_{r}^{\mathrm{N}^{*}}$ is a convex function of $c_{p}$. Then, there are two roots (with respect to $c_{p}$ ) for the equation $\pi_{r}^{\mathrm{EB}^{*}}-\pi_{r}^{\mathrm{N}^{*}}=0: c_{p}^{*}=\frac{16 c_{r}+19 k+21 c_{r} k+6 c_{r} k^{2}+2 k^{2}+18}{8 k^{2}+40 k+34}$ and $c_{p}^{* *}=\frac{84 c_{r}-159 k+199 c_{r} k+146 c_{r} k^{2}+32 c_{r} k^{3}-138 k^{2}-32 k^{3}-50}{8 k^{2}+40 k+34}$. This, together with the fact that $c_{p}^{* *}<\underline{\underline{c_{p}}}<\overline{\overline{c_{p}}}<c_{p}^{*}$, suggests that, for any $c_{p}, \pi_{r}^{\mathrm{EB}^{*}}-\pi_{r}^{\mathrm{N}^{*}}<0$ always holds.

Proof of Proposition 5.3. Based on the outcomes of Lemmas A.2 and A.3, we compare the supplier's profits between Model EB and Model ET:

$\pi_{s}^{\mathrm{EB}^{*}}-\pi_{s}^{\mathrm{ET}^{*}}=\frac{\left[\begin{array}{l}-\left(512 c_{p}^{2} k^{6}+3328 c_{p}^{2} k^{5}+7720 c_{p}^{2} k^{4}+8168 c_{p}^{2} k^{3}+3630 c_{p}^{2} k^{2}+68 c_{p}^{2} k+289 c_{p}^{2}+256 c_{p} c_{r} k^{5}\right. \\ +1544 c_{p} c_{r} k^{4}+3644 c_{p} c_{r} k^{3}+4344 c_{p} c_{r} k^{2}+2640 c_{p} c_{r} k+646 c_{p} c_{r}-1024 c_{p} k^{6}-6912 c_{p} k^{5} \\ -16984 c_{p} k^{4}-19980 c_{p} k^{3}-11604 c_{p} k^{2}-2776 c_{p} k-68 c_{p}+8 c c_{r}^{2} k^{5}+24 c_{r}^{2} k^{4}-36 c_{r}^{2} k^{3} \\ -190 c_{r}^{2} k^{2}-212 c_{r}^{2} k-73 c_{r}^{2}-272 c_{r} k^{5}-1592 c_{r} k^{4}-3572 c_{r} k^{3}-3964 c_{r} k^{2}-2216 c_{r} k-500 c_{r} \\ \left.+512 k^{6}+3592 k^{5}+9288 k^{4}+11776 k^{3}+7784 k^{2}+2496 k+284\right)\end{array}\right]}{4(4 k+3)\left(16 k^{3}+70 k^{2}+89 k+34\right)^{2}}$.

We find that $\pi_{s}^{\mathrm{EB}^{*}}-\pi_{s}^{\mathrm{ET}}$ is a quadratic function of $c_{p}$, and the coefficient $\frac{-\left(1024 k^{6}+6656 k^{5}+15440 k^{4}+16336 k^{3}+7260 k^{2}+136 k+578\right)}{4(4 k+3)\left(16 k^{3}+70 k^{2}+89 k+34\right)^{2}}$ is negative, which shows that the equation of $\pi_{s}^{\mathrm{EB}^{*}}-\pi_{s}^{\mathrm{ET}^{*}}$ is a concave function of $c_{p}$. Further, solving $\pi_{s}^{\mathrm{EB}}-\pi_{s}^{\mathrm{ET}}=0$, we obtain that there exist two roots (with respect to $\left.c_{p}\right)$ :

$$
c_{p}^{* *}=\frac{\left[\begin{array}{l}
256 k-86 c_{r}+2\left(( k + 1 ) ^ { 3 } \left(1024 k^{7}+7552 k^{6}+22976 k^{5}+37108 k^{4}+34037 k^{3}+17525 k^{2}+4629 k\right.\right. \\
+477))^{\frac{1}{2}}-292 c_{r} k-376 c_{r} k^{2}-218 c_{r} k^{3}-48 c_{r} k^{4}+664 k^{2}+846 k^{3}+528 k^{4}+128 k^{5}-2 c_{r}\left((k+1)^{3}\right. \\
\left.\left(1024 k^{7}+7552 k^{6}+22976 k^{5}+37108 k^{4}+34037 k^{3}+17525 k^{2}+4629 k+477\right)\right)^{\frac{1}{2}}+37
\end{array}\right]}{128 k^{5}+480 k^{4}+628 k^{3}+288 k^{2}-36 k+5}
$$

and

$$
c_{p}^{*}=\frac{\left[\begin{array}{l}
256 k-86 c_{r}-2\left(( k + 1 ) ^ { 3 } \left(1024 k^{7}+7552 k^{6}+22976 k^{5}+37108 k^{4}+34037 k^{3}+17525 k^{2}+4629 k\right.\right. \\
+477))^{\frac{1}{2}}-292 c_{r} k-376 c_{r} k^{2}-218 c_{r} k^{3}-48 c_{r} k^{4}+664 k^{2}+846 k^{3}+528 k^{4}+128 k^{5}+2 c_{r}\left((k+1)^{3}\right. \\
\left.\left(1024 k^{7}+7552 k^{6}+22976 k^{5}+37108 k^{4}+34037 k^{3}+17525 k^{2}+4629 k+477\right)\right)^{\frac{1}{2}}+37
\end{array}\right]}{128 k^{5}+480 k^{4}+628 k^{3}+288 k^{2}-36 k+5} .
$$

This, together with the fact that $c_{p}^{*}<\underline{\underline{c_{p}}}<\overline{\overline{c_{p}}}<c_{p}^{* *}$, suggests that, for any $c_{p}, \pi_{s}^{\mathrm{EB}^{*}}-\pi_{s}^{\mathrm{ET}^{*}}>0$ always holds. 
Next, we compare the traditional retailer's profits between Model EB and Model ET:

$$
\pi_{r}^{\mathrm{EB}^{*}}-\pi_{r}^{\mathrm{ET}^{*}}=\frac{\left[\begin{array}{l}
(k+1)\left(704 c_{p}^{2} k^{4}+4108 c_{p}^{2} k^{3}+7528 c_{p}^{2} k^{2}+5559 c_{p}^{2} k+1445 c_{p}^{2}-1536 c_{p} c_{r} k^{5}-9776 c_{p} c_{r} k^{4}\right. \\
-23296 c_{p} c_{r} k^{3}-26404 c_{p} c_{r} k^{2}-14372 c_{p} c_{r} k-3026 c_{p} c_{r}+1536 c_{p} k^{5}+8368 c_{p} k^{4}+15080 c_{p} k^{3} \\
+11348 c_{p} k^{2}+3254 c_{p} k+136 c_{p}+256 c_{r}^{2} k^{6}+2160 c_{r}^{2} k^{5}+7232 c_{r}^{2} k^{4}+12304 c_{r}^{2} k^{3}+11268 c_{r}^{2} k^{2} \\
+5293 c_{r}^{2} k+1001 c_{r}^{2}-512 c_{r} k^{6}-2784 c_{r} k^{5}-4688 c_{r} k^{4}-1312 c_{r} k^{3}+3868 c_{r} k^{2}+3786 c_{r} k \\
\left.+1024 c_{r}+256 k^{6}+624 k^{5}-1840 k^{4}-6884 k^{3}-7608 k^{2}-3520 k-580\right)
\end{array}\right]}{4\left(64 k^{4}+328 k^{3}+566 k^{2}+403 k+102\right)^{2}} .
$$

$\pi_{r}^{\mathrm{EB}^{*}}-\pi_{r}^{\mathrm{ET}} \mathrm{T}^{*}$ is a quadratic function of $c_{p}$, and the coefficient of the quadratic function is $\frac{704 k^{5}+4812 k^{4}+11636 k^{3}+13087 k^{2}+7004 k+1445}{2\left(64 k^{4}+328 k^{3}+566 k^{2}+403 k+102\right)^{2}}$. Further, solving $\pi_{r}^{\mathrm{EB}^{*}}-\pi_{r}^{\mathrm{ET}^{*}}=0$, we obtain that there exist two roots (with respect to $c_{p}$ ):

$$
c_{p}^{* *}=\frac{7 c_{r}+10 k+12 c_{r} k+4 c_{r} k^{2}-4 k^{2}+10}{22 k+17} \quad \text { and } \quad c_{p}^{*}=\frac{\left[\begin{array}{l}
143 c_{r}-294 k+511 c_{r} k+652 c_{r} k^{2}+348 c_{r} k^{3} \\
+64 c_{r} k^{4}-490 k^{2}-316 k^{3}-64 k^{4}-58
\end{array}\right]}{32 k^{3}+162 k^{2}+217 k+85} .
$$

After calculation, we can find that, $c_{p}^{*}<\underline{\underline{c_{p}}}<\overline{\overline{c_{p}}}<c_{p}^{* *}$. Hence, for any $c_{p}, \pi_{r}^{\mathrm{EB}^{*}}-\pi_{r}^{\mathrm{ET}} \mathrm{T}^{*}<0$ always holds.

Additionally, we compare the platform owner's profits between Model EB and Model ET:

$$
\begin{aligned}
& \pi_{P}^{\mathrm{EB}^{*}}-\pi_{P}^{\mathrm{ET}}= \\
& {\left[\begin{array}{l}
3072 c_{p}^{2} k^{7}+24832 c_{p}^{2} k^{6}+80624 c_{p}^{2} k^{5}+139264 c_{p}^{2} k^{4}+139308 c_{p}^{2} k^{3}+80744 c_{p}^{2} k^{2}+25024 c_{p}^{2} k \\
+3179 c_{p}^{2}-1024 c_{p} c_{r} k^{7}-7936 c_{p} c_{r} k^{6}-25328 c_{p} c_{r} k^{5}-43168 c_{p} c_{r} k^{4}-41956 c_{p} c_{r} k^{3}-22756 c_{p} c_{r} k^{2} \\
-6136 c_{p} c_{r} k-578 c_{p} c_{r}-5120 c_{p} k^{7}-41728 c_{p} k^{6}-135920 c_{p} k^{5}-235360 c_{p} k^{4}-236660 c_{p} k^{3} \\
-138732 c_{p} k^{2}-43912 c_{p} k-5780 c_{p}+64 c_{r}^{2} k^{6}+348 c_{r}^{2} k^{5}+576 c_{r}^{2} k^{4}+92 c_{r}^{2} k^{3}-628 c_{r}^{2} k^{2}-584 c_{r}^{2} k \\
-157 c_{r}^{2}+1024 c_{r} k^{7}+7808 c_{r} k^{6}+24632 c_{r} k^{5}+42016 c_{r} k^{4}+41772 c_{r} k^{3}+24012 c_{r} k^{2}+7304 c_{r} k \\
+892 c_{r}+2048 k^{7}+16960 k^{6}+55644 k^{5}+96672 k^{4}+97444 k^{3}+57360 k^{2}+18304 k+2444
\end{array}\right]} \\
& \frac{4\left(64 k^{4}+328 k^{3}+566 k^{2}+403 k+102\right)^{2}}{}
\end{aligned}
$$

$\pi_{p}^{\mathrm{EB}^{*}}-\pi_{p}^{\mathrm{ET}}{ }^{*}$ is a quadratic function of $c_{p}$, and the coefficient $\frac{\left[\begin{array}{c}6144 k^{7}+49664 k^{6}+161248 k^{5}+278528 k^{4} \\ +278616 k^{3}+161488 k^{2}+50048 k+6358\end{array}\right]}{4\left(64 k^{4}+328 k^{3}+566 k^{2}+403 k+102\right)^{2}}$ is positive. That means the equation of $\pi_{p}^{\mathrm{EB}}-\pi_{p}^{\mathrm{ET}}{ }^{*}$ is a convex function of $c_{p}$. Further, solving $\pi_{p}^{\mathrm{EB}}{ }^{*}-\pi_{p}^{\mathrm{ET}}=0$, we obtain that there exist two roots (with respect to $c_{p}$ ):

$$
c_{p}^{*}=\frac{\left[\begin{array}{l}
289 c_{r}+21956 k+204 c_{r}\left((k+1)^{3}\left(16 k^{3}+24 k^{2}+29 k+14\right)\right)^{(1 / 2)}+3068 c_{r} k-806 k\left((k+1)^{3}\right. \\
\left.\left(16 k^{3}+24 k^{2}+29 k+14\right)\right)^{(1 / 2)}-204\left((k+1)^{3}\left(16 k^{3}+24 k^{2}+29 k+14\right)\right)^{(1 / 2)}+11378 c_{r} k^{2} \\
+20978 c_{r} k^{3}+21584 c_{r} k^{4}+12664 c_{r} k^{5}+3968 c_{r} k^{6}+512 c_{r} k^{7}-1132 k^{2}\left(( k + 1 ) ^ { 3 } \left(16 k^{3}+24 k^{2}\right.\right. \\
+29 k+14))^{(1 / 2)}-656 k^{3}\left((k+1)^{3}\left(16 k^{3}+24 k^{2}+29 k+14\right)\right)^{(1 / 2)}-128 k^{4}\left(( k + 1 ) ^ { 3 } \left(16 k^{3}+24 k^{2}\right.\right. \\
+29 k+14))^{(1 / 2)}+69366 k^{2}+118330 k^{3}+117680 k^{4}+67960 k^{5}+20864 k^{6}+2560 k^{7}+2890 \\
+806 c_{r} k\left((k+1)^{3}\left(16 k^{3}+24 k^{2}+29 k+14\right)\right)^{(1 / 2)}+1132 c_{r} k^{2}\left((k+1)^{3}\left(16 k^{3}+24 k^{2}+29 k+14\right)\right)^{(1 / 2)} \\
+656 c_{r} k^{3}\left((k+1)^{3}\left(16 k^{3}+24 k^{2}+29 k+14\right)\right)^{(1 / 2)}+128 c_{r} k^{4}\left((k+1)^{3}\left(16 k^{3}+24 k^{2}+29 k+14\right)\right)^{(1 / 2)}
\end{array}\right]}{3072 k^{7}+24832 k^{6}+80624 k^{5}+139264 k^{4}+139308 k^{3}+80744 k^{2}+25024 k+3179}
$$


and

$c_{p}^{* *}=\frac{\left[\begin{array}{l}289 c_{r}+21956 k-204 c_{r}\left((k+1)^{3}\left(16 k^{3}+24 k^{2}+29 k+14\right)\right)^{(1 / 2)}+3068 c_{r} k+806 k\left((k+1)^{3}\right. \\ \left.\left(16 k^{3}+24 k^{2}+29 k+14\right)\right)^{(1 / 2)}-204\left((k+1)^{3}\left(16 k^{3}+24 k^{2}+29 k+14\right)\right)^{(1 / 2)}+11378 c_{r} k^{2} \\ +20978 c_{r} k^{3}+21584 c_{r} k^{4}+12664 c_{r} k^{5}+3968 c_{r} k^{6}+512 c_{r} k^{7}+1132 k^{2}\left((k+1)^{3}\left(16 k^{3}+24 k^{2}\right.\right. \\ +29 k+14))^{(1 / 2)}+656 k^{3}\left((k+1)^{3}\left(16 k^{3}+24 k^{2}+29 k+14\right)\right)^{(1 / 2)}+128 k^{4}\left((k+1)^{3}\left(16 k^{3}+24 k^{2}\right.\right. \\ +29 k+14))^{(1 / 2)}+69366 k^{2}+118330 k^{3}+117680 k^{4}+67960 k^{5}+20864 k^{6}+2560 k^{7}+2890 \\ -806 c_{r} k\left((k+1)^{3}\left(16 k^{3}+24 k^{2}+29 k+14\right)\right)^{(1 / 2)}-1132 c_{r} k^{2}\left((k+1)^{3}\left(16 k^{3}+24 k^{2}+29 k+14\right)\right)^{(1 / 2)} \\ -656 c_{r} k^{3}\left((k+1)^{3}\left(16 k^{3}+24 k^{2}+29 k+14\right)\right)^{(1 / 2)}-128 c_{r} k^{4}\left((k+1)^{3}\left(16 k^{3}+24 k^{2}+29 k+14\right)\right)^{(1 / 2)}\end{array}\right.}{3072 k^{7}+24832 k^{6}+80624 k^{5}+139264 k^{4}+139308 k^{3}+80744 k^{2}+25024 k+3179}$.

To simplify the comparison, we can obtain that, $\underline{\underline{c_{p}}}<\overline{\overline{c_{p}}}<c_{p}^{*}<c_{p}^{* *}$. Thus, we can conclude that, for any $c_{p}$, $\pi_{p}^{\mathrm{EB}^{*}}-\pi_{p}^{\mathrm{ET}^{*}}>0$ always holds.

Acknowledgements. This research is supported by the National Natural Science Foundation of China (Nos. 71971043), Major Program of National Social Science Foundation of China (No. 20\&ZD084), National Natural Science Foundation of China (No.72072020 and No.71672020).

\section{REFERENCES}

[1] M. Armstrong, Competition in two-sided markets. RAND J. Econ. 37 (2006) 668-691.

[2] A. Arya and B. Mittendorf, The changing face of distribution channels: partial forward integration and strategic investments. Prod. Oper. Manag. 22 (2013) 1077-1088.

[3] A. Arya, B. Mittendorf and D.E.M. Sappington, The bright side of supplier encroachment. Mark. Sci. 26 (2007) 651-659.

[4] P. Belleflamme and M. Peitz, Price disclosure by two-sided platforms. Int. J. Ind. Organ. 67 (2019) 102529.

[5] K. Boudreau, Open platform strategies and innovation: granting access vs. Devolving control. Manage. Sci. 56 (2010) 18491872.

[6] M. Brandenburg and T. Rebs, Sustainable supply chain management: a modeling perspective. Ann. Oper. Res. 229 (2015) 213-252.

[7] K. Cattani, W. Gilland, H.S. Heese and J. Swaminathan, Boiling frogs: pricing strategies for a manufacturer adding a direct channel that competes with the traditional channel. Prod. Oper. Manag. 15 (2006) 40-56.

[8] J. Chai, H. Li, W. Yan and Y. Li, Should a retailer sell its own extended warranties or resell those from the manufacturer when confronting supplier encroachment? J. Oper. Res. Soc. 72 (2020) 2046-2058.

[9] J. Chai, W. Yan, Y. Li, M. Palmer and Q. Huang, Selling vertically differentiated products under one channel or two? A quality segmentation model for differentiated distribution channels. J. Oper. Res. Soc. 71 (2020) 1180-1198.

[10] F. Chen, Salesforce incentives, market information, and production/inventory planning. Manage. Sci. 51 (2005) 60-75.

[11] P. Chen, B. Li and H. Huang, Decision policies on players' different risk combination under supplier encroachment. RAIROOper. Res. 54 (2020) 1057-1075.

[12] J. Chen, W. Zhang and Z. Liu, Joint pricing, services and quality decisions in a dual-channel supply chain. RAIRO-Oper. Res. 54 (2020) 1041-1056.

[13] W.Y.K. Chiang, D. Chhajed and J.D. Hess, Direct-marketing, indirect profits: a strategic analysis of dual-channel supply-chain design. Manage. Sci. 49 (2003) 1-20.

[14] J. Chu and P. Manchanda, Quantifying cross and direct network effects in online consumer-to-consumer platforms. Mark. Sci. 35 (2016) 870-893.

[15] A.T. Coughlan, Chapter 13 salesforce compensation: a review of MS/OR advances. Handb. Oper. Res. Manage. Sci. 5 (1993) 611-651.

[16] P.S. Desai, Quality segmentation in spatial markets: when does cannibalization affect product line design? Mark. Sci. 20 (2001) 265-283.

[17] A. Ha, X. Long and J. Nasiry, Quality in supply chain encroachment. MESSOM-Manuf. Serv. Oper. Manag. 18 (2016) $280-298$.

[18] A. Hagiu, Merchant or two-sided platform? Rev. Netw. Econ. 6 (2007) 115-133.

[19] A. Haviv, Y. Huang and N. Li, Intertemporal demand spillover effects on video game platforms. Manage. Sci. 66 (2020) 4788-4807. 
[20] B. Jiang, K. Jerath and K. Srinivasan, Firm strategies in the "mid tail" of platform-based retailing. Mark. Sci. 30 (2011) $757-775$.

[21] J. Li and Y. Zhang, More market awareness, more profit? Competitive environments, business expansions, and two-sided markets. Manag. Decis. Econ. 42 (2021) 249-267.

[22] K. Li, S. Mallik and D. Chhajed, Design of extended warranties in supply chains under additive demand. Prod. Oper. Manag. 21 (2012) 730-746.

[23] Z. Li, S.M. Gilbert and G. Lai, Supplier encroachment under asymmetric information. Manage. Sci. 60 (2013) 449-462.

[24] Z. Li, S.M. Gilbert and G. Lai, Supplier encroachment as an enhancement or a hindrance to nonlinear pricing. Prod. Oper. Manag. 24 (2015) 89-109.

[25] M. Lin, S. Li and A. Whinston, Innovation and price competition in a two-sided market. J. Manage. Inform. Syst. 28 (2011) 171-202.

[26] Y. Liu, S. Gupta and Z.J. Zhang, Note on self-restraint as an online entry-deterrence strategy. Manage. Sci. 52 (2006) 17991809.

[27] B. Mantin, H. Krishnan and T. Dhar, The strategic role of third-party marketplaces in retailing. Prod. Oper. Manag. 23 (2014) 1937-1949.

[28] D.P. McIntyre and A. Srinivasan, Networks, platforms, and strategy: emerging views and next steps. Strateg. Manage. J. 38 (2017) 141-160.

[29] S.K. Mukhopadhyay, X. Su and S. Ghose, Motivating retail marketing effort: optimal contract design. Prod. Oper. Manag. 18 (2009) 197-211.

[30] E. Ofek, Z. Katona and M. Sarvary, "Bricks and Clicks": the impact of product returns on the strategies of multichannel retailers. Mark. Sci. 30 (2011) 42-60.

[31] K.F. Park, R. Seamans and F. Zhu, Homing and platform responses to entry: historical evidence from the U.S. Newspaper industry. Strateg. Manage. J. 42 (2021) 684-709.

[32] J.-C. Rochet and J. Tirole, Platform competition in two-sided markets. J. Eur. Econ. Assoc. 1 (2003) $990-1029$.

[33] R.C. Savaskan, S. Bhattacharya and L.N.V. Wassenhove, Closed-loop supply chain models with product remanufacturing. Manage. Sci. 50 (2004) 239-252.

[34] S. Sriram, P. Manchanda, M.E. Bravo, J. Chu, L. Ma, M. Song, S. Shriver and U. Subramanian, Platforms: a multiplicity of research opportunities. Mark. Lett. 26 (2015) 141-152.

[35] Statista, Percentage of paid units sold by third-party sellers on amazon platform as of 4th quarter 2016. Available at: http: //dx.doi.org/10.1111/j.1937-5956.2004.tb00147.x (2017).

[36] X. Sun, W. Tang, J. Chen, S. Li and J. Zhang, Manufacturer encroachment with production cost reduction under asymmetric information. Transp. Res. Pt. e-Logist. Transp. Rev. 128 (2019) 191-211.

[37] L. Sun, R. H. Teunter, M.Z. Babai and G. Hua, Optimal pricing for ride-sourcing platforms. Eur. J. Oper. Res. 278 (2019) $783-795$.

[38] A.A. Tsay and N. Agrawal, Channel conflict and coordination in the e-commerce age. Prod. Oper. Manag. 13 (2004) 93-110.

[39] R.K. Tyagi, Technological advances, transaction costs, and consumer welfare. Mark. Sci. 23 (2004) 335-344.

[40] Y. Xiong, W. Yan, K. Fernandes, Z.-K. Xiong and N. Guo, "Bricks vs. Clicks": The impact of manufacturer encroachment with a dealer leasing and selling of durable goods. Eur. J. Oper. Res. 217 (2012) 75-83.

[41] D.-H. Yoon, Supplier encroachment and investment spillovers. Prod. Oper. Manag. 25 (2016) 1839-1854.

\section{Subscribe to Open (S20) A fair and sustainable open access model}

This journal is currently published in open access under a Subscribe-to-Open model (S2O). S2O is a transformative model that aims to move subscription journals to open access. Open access is the free, immediate, online availability of research articles combined with the rights to use these articles fully in the digital environment. We are thankful to our subscribers and sponsors for making it possible to publish this journal in open access, free of charge for authors.

\section{Please help to maintain this journal in open access!}

Check that your library subscribes to the journal, or make a personal donation to the S2O programme, by contacting subscribers@edpsciences.org

More information, including a list of sponsors and a financial transparency report, available at: https://www. edpsciences.org/en/maths-s2o-programme 